\title{
Collaboration of Trinuclear Aluminum Complexes Bearing Bipyrazoles in Ring-Opening Polymerization of $\varepsilon$-Caprolactone
}

Someswara Rao Kosuru, ${ }^{a}$ Feng-Jie Lai, ${ }^{b c} *$ Yu-Lun Chang, ${ }^{a}$ Chen-Yu Li, ${ }^{a}$ Yi-Chun Lai, ${ }^{a}$ Shangwu Ding, ${ }^{a d}$ Kuo-Hui Wu*, ${ }^{e}$ Hsuan-Ying Chen*, adf Yung-Han Lo ${ }^{g}$

a Department of Medicinal and Applied Chemistry, Drug Development and Value Creation Research Center, Kaohsiung Medical University, Kaohsiung, Taiwan, 80708, R.O.C.

${ }^{b}$ Department of Dermatology, Chi Mei Medical Center, Tainan, Taiwan, R.O.C.

c Center for General Education, Southern Taiwan University of Science and Technology, Tainan, Taiwan, R.O.C.

${ }^{d}$ Department of Chemistry, National Sun Yat-Sen University, Kaohsiung, Taiwan, 80424, R.O.C.

e Department of Chemistry, National Central University, Taoyuan, 32001, Taiwan

${ }^{f}$ Department of Medical Research, Kaohsiung Medical University Hospital, Kaohsiung 80708, Taiwan, R.O.C.

$g$ Department of Chemistry, Faculty of Science and Technology, Keio University, Minato City, 108-8345, Tokyo

Supporting Information

Table of Contents

Table S1. Kinetic study of CL polymerization with various trinuclear Al complexes ${ }^{a}$ 4

Table S2. Kinetic study of CL polymerization with various concentration of $\mathrm{D}^{\mathrm{Bu}}{ }_{2} \mathrm{Al}_{3} \mathrm{Me}_{5}$ and $\mathrm{BnOH}$ in toluene $5 \mathrm{~mL},[\mathrm{CL}]=2.0 \mathrm{M}$ at room temperature ${ }^{a}$

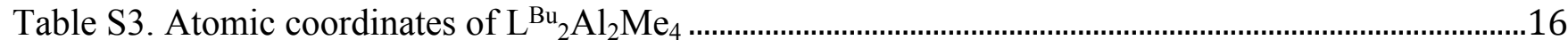

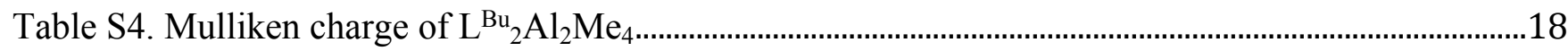

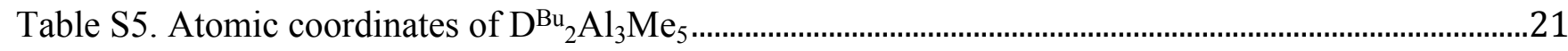

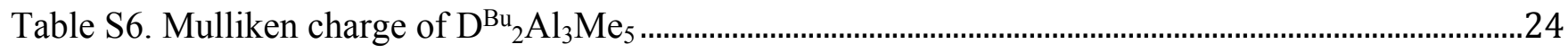

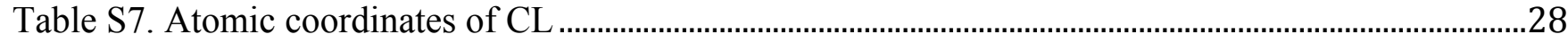

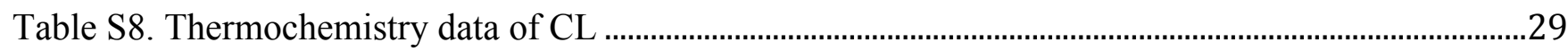

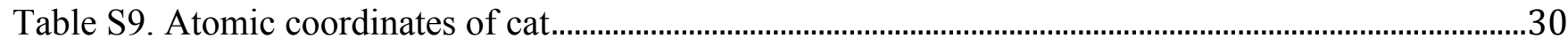

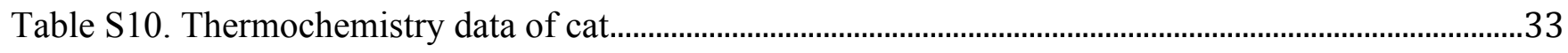

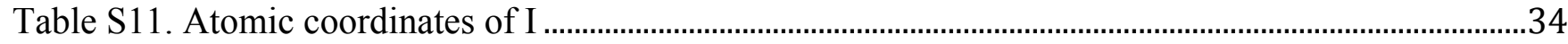

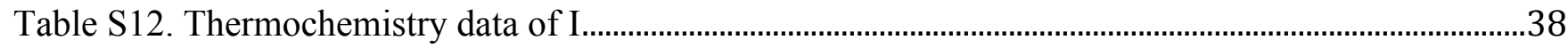

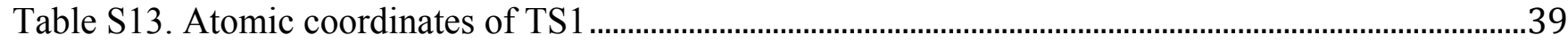




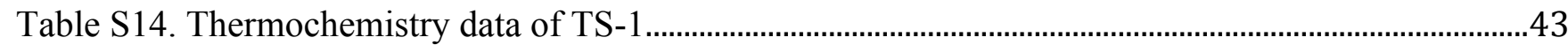

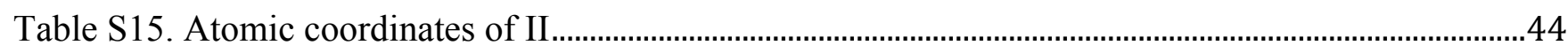

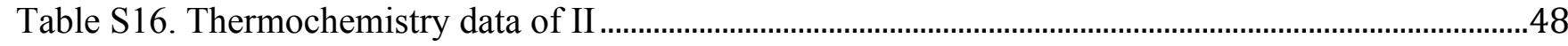

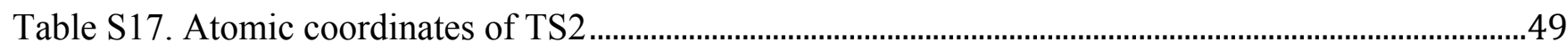

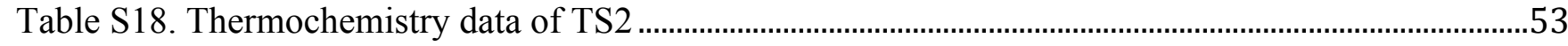

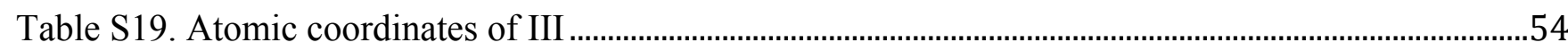

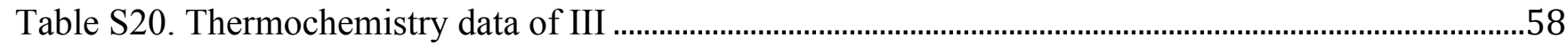

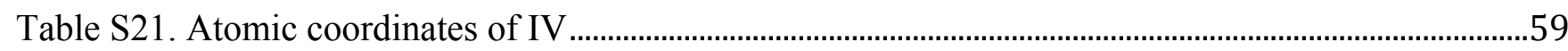

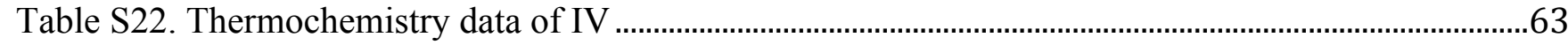

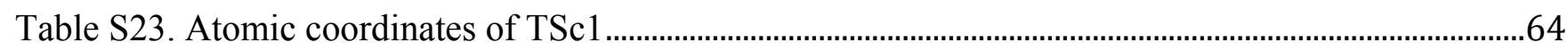

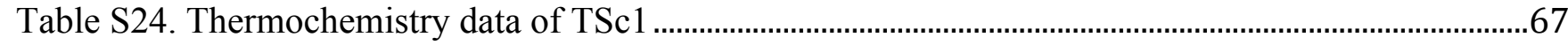

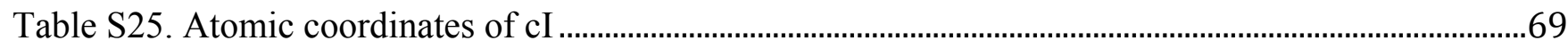

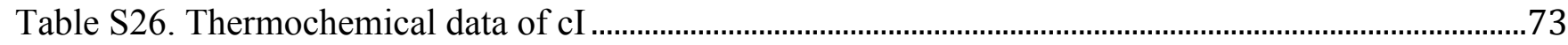

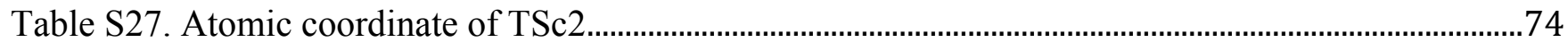

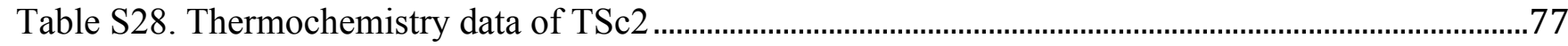

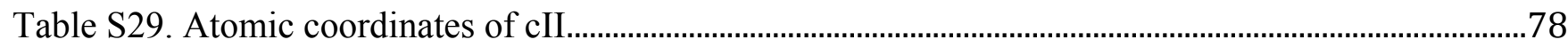

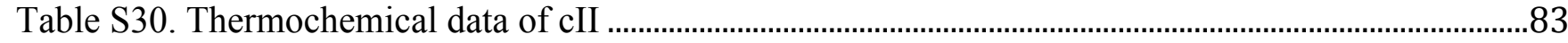

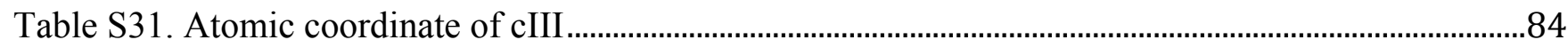

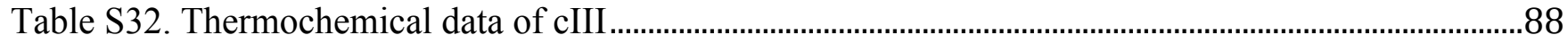

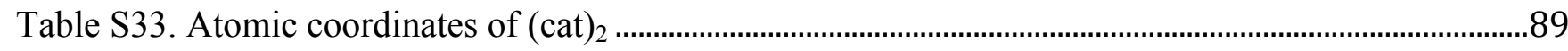

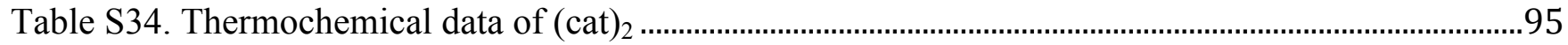

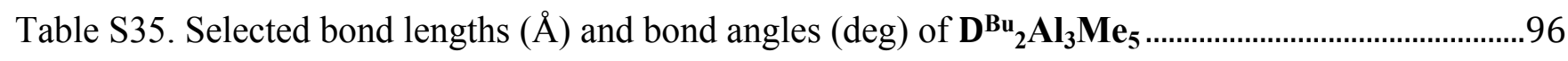

Figure S1. First-order kinetic plots of CL polymerization with various concentrations of [trinuclear Al 
complexes $\cdot 5 \mathrm{BnOH}]$ plotted against time with $[\mathrm{CL}]=2.0 \mathrm{M}$ in toluene $5 \mathrm{~mL}$ (Table 2). 5

Figure S2. First-order kinetic plots of CL polymerization with various concentrations of $\left[\mathrm{D}^{\mathrm{Bu}_{2}} \mathrm{Al}_{3} \mathrm{Me}_{5} \cdot 5 \mathrm{BnOH}\right]$ plotted against time with $[\mathrm{CL}]=2.0 \mathrm{M}$ in toluene $5 \mathrm{~mL}$ .7

Figure S3. Linear plot of $k_{\text {obs }}$ versus $\left[\mathrm{D}^{\mathrm{Bu}}{ }_{2} \mathbf{A l}_{3} \mathbf{M e}_{5} \cdot 5 \mathrm{BnOH}\right]$ for the polymerization of $\mathrm{CL}$ with $[\mathrm{CL}]=$

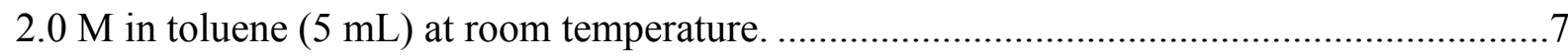

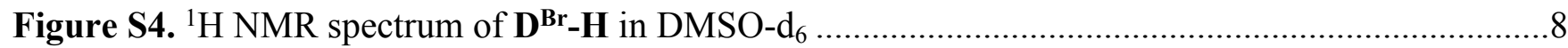

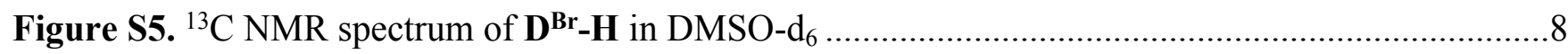

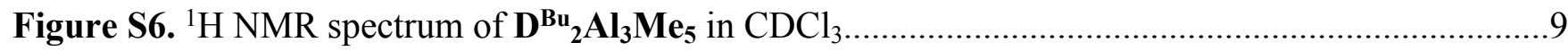

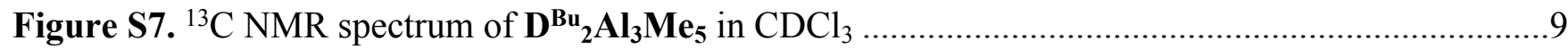

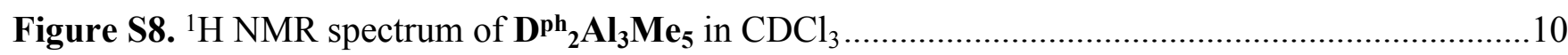

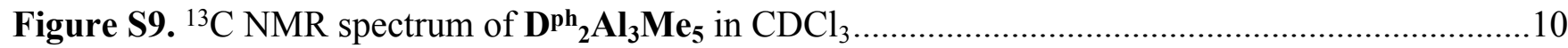

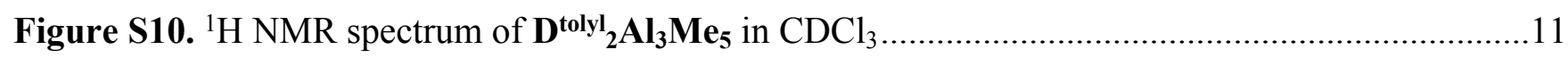

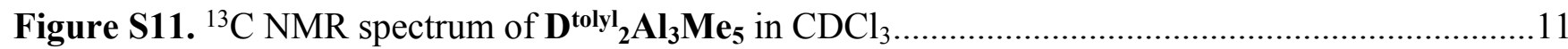

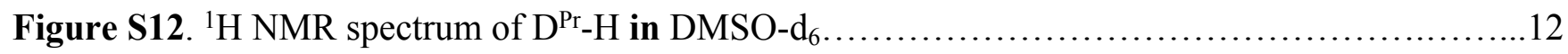

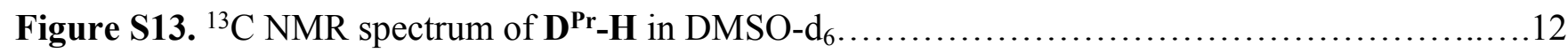

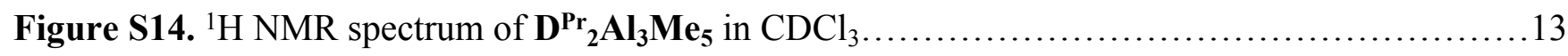

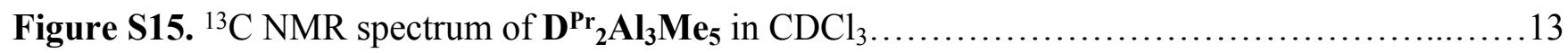

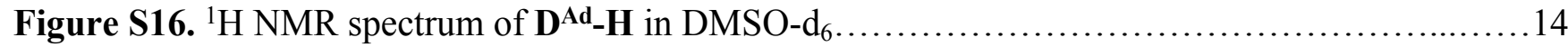

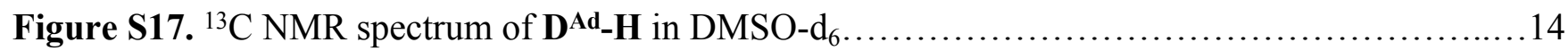

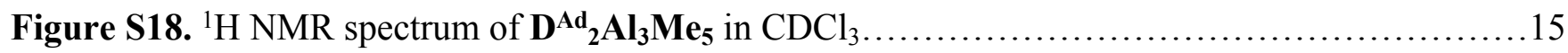

Figure S19. ${ }^{13} \mathrm{C}$ NMR spectrum of $\mathbf{D}^{\mathbf{A d}_{2} \mathbf{A l}_{3} \mathbf{M e}_{5} \text { in } \mathrm{CDCl}_{3} \ldots \ldots \ldots \ldots \ldots \ldots \ldots \ldots \ldots \ldots \ldots \ldots \ldots \ldots \ldots \ldots \ldots \ldots \ldots}$ 
Table S1. Kinetic study of CL polymerization with various trinuclear Al complexes ${ }^{a}$

\begin{tabular}{|c|c|c|c|c|c|}
\hline & \multicolumn{5}{|c|}{$[\mathrm{CL}]:[\mathrm{Cat}]:[\mathrm{BnOH}]$} \\
\hline & \multicolumn{5}{|c|}{$100: 0.5: 2.5$} \\
\hline \multirow[t]{2}{*}{ Time/min } & $\mathrm{D}^{\mathrm{Pr}_{2}} \mathrm{Al}_{3} \mathrm{Me}_{5}$ & $\mathrm{D}^{\mathrm{Ad}}{ }_{2} \mathrm{Al}_{3} \mathbf{M e}_{5}$ & $\mathrm{D}^{\mathrm{Bu}}{ }_{2} \mathrm{Al}_{3} \mathrm{Me}_{5}$ & $\mathrm{D}^{\mathrm{ph}}{ }_{2} \mathrm{Al}_{3} \mathrm{Me}_{5}$ & $D^{\text {tolyl }}{ }_{2} \mathrm{Al}_{3} \mathbf{M e}_{5}$ \\
\hline & \multicolumn{5}{|c|}{ PCL conversion ${ }^{b}$} \\
\hline 5 & & 0.25 & & & \\
\hline 6 & & & 0.33 & & \\
\hline 7 & & 0.52 & & & \\
\hline 8 & & & & & \\
\hline 9 & & & 0.6 & & \\
\hline 10 & & 0.71 & & & \\
\hline 12 & & & 0.78 & & \\
\hline 13 & & 0.85 & & & \\
\hline 15 & & & 0.87 & & 0.16 \\
\hline 16 & & 0.92 & & & \\
\hline 18 & & & 0.93 & & \\
\hline 20 & 0.13 & & & 0.26 & 0.35 \\
\hline 30 & 0.42 & & & 0.56 & \\
\hline 35 & & & & & 0.7 \\
\hline 40 & 0.67 & & & 0.76 & \\
\hline 45 & & & & & 0.82 \\
\hline 50 & 0.81 & & & 0.86 & \\
\hline 55 & & & & 0.9 & 0.9 \\
\hline 60 & 0.89 & & & & \\
\hline $\begin{array}{c}\mathrm{k}_{\mathrm{obs}} \times 10^{2} / \\
\min ^{-1} \\
(\text { error })\end{array}$ & $5.25(17)$ & $20.46(36)$ & $\begin{array}{c}18.81 \\
(32)\end{array}$ & 5.71 & 5.28 \\
\hline $\begin{array}{l}\text { I.P/ min } \\
\text { (error) }\end{array}$ & $18(1)$ & $4(1)$ & $4(1)$ & $15(1)$ & $12(1)$ \\
\hline
\end{tabular}

${ }^{a}$ In general, the reaction was carried out in toluene with $[\mathbf{C L}]=2.0 \mathrm{M}$ at $25^{\circ} \mathrm{C}$.

${ }^{b}$ The data were determined from ${ }^{1} \mathrm{H}$ NMR analysis. 


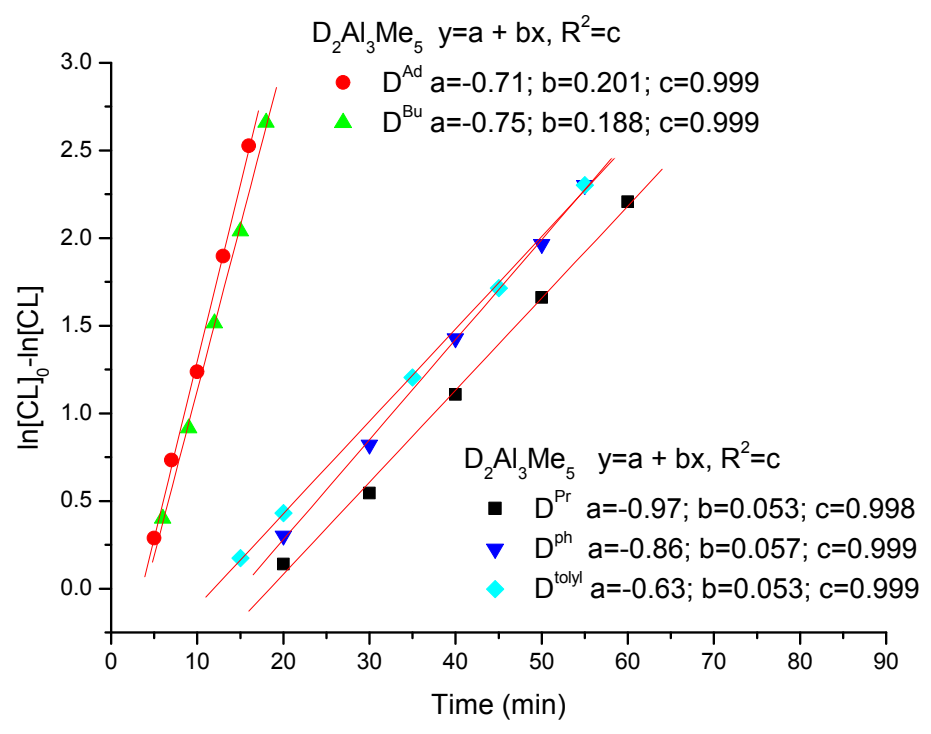

Figure S1. First-order kinetic plots of CL polymerization with various concentrations of [trinuclear $\mathrm{Al}$ complexes $\cdot 5 \mathrm{BnOH}$ ] plotted against time with $[\mathrm{CL}]=2.0 \mathrm{M}$ in toluene $5 \mathrm{~mL}$ (Table 2). 
Table S2. Kinetic study of CL polymerization with various concentration of $\mathbf{D}^{\mathbf{B u}_{2}} \mathbf{A l}_{3} \mathbf{M e}_{5}$ and $\mathrm{BnOH}$ in toluene $5 \mathrm{~mL},[\mathrm{CL}]=2.0 \mathrm{M}$ at room temperature ${ }^{a}$

\begin{tabular}{|c|c|c|c|c|c|c|}
\hline & \multicolumn{6}{|c|}{$[\mathrm{CL}]:\left[\mathbf{D}^{\mathrm{Bu}}{ }_{2} \mathbf{A l}_{3} \mathbf{M e}_{5}\right]:[\mathrm{BnOH}]$} \\
\hline time & $100: 1: 1$ & $100: 1: 3$ & $100: 1: 5$ & $100: 0.75: 3.75$ & $100: 0.5: 2.5$ & $100: 0.25: 1.25$ \\
\hline $\min$ & \multicolumn{6}{|c|}{ PCL conversion ${ }^{b}$} \\
\hline 2 & & & 0.15 & & & \\
\hline 3 & & & 0.36 & & & \\
\hline 4 & & 0.34 & 0.52 & 0.34 & & \\
\hline 6 & & & 0.72 & 0.56 & 0.33 & \\
\hline 8 & & 0.51 & & 0.69 & & \\
\hline 9 & & & & & 0.6 & \\
\hline 12 & & & 0.95 & 0.87 & 0.78 & \\
\hline 15 & & & & 0.93 & 0.87 & \\
\hline 18 & & & & & 0.93 & \\
\hline 19 & & 0.76 & & & & \\
\hline 21 & & & & & & 0.35 \\
\hline 25 & & 0.84 & & & & \\
\hline 32 & 0.37 & & & & & \\
\hline 35 & & 0.92 & & & & 0.61 \\
\hline 45 & & & & & & 0.72 \\
\hline 60 & & & & & & 0.85 \\
\hline 70 & & & & & & 0.9 \\
\hline 90 & 0.63 & & & & & \\
\hline 110 & 0.69 & & & & & \\
\hline 190 & 0.84 & & & & & \\
\hline 240 & 0.9 & & & & & \\
\hline $\begin{array}{l}\mathrm{k}_{\mathrm{obs}} \times \quad 10^{2} / \\
\min ^{-1} \text { (error) }\end{array}$ & $0.87(1)$ & $6.75(6)$ & $\begin{array}{r}28.30 \\
(16)\end{array}$ & $\begin{array}{c}20.46 \\
(33)\end{array}$ & $\begin{array}{c}18.81 \\
(32)\end{array}$ & $\begin{array}{c}3.82 \\
(9)\end{array}$ \\
\hline $\begin{array}{l}\text { I.P/ } \\
\min (\text { error) }\end{array}$ & 0 & 0 & $1(1)$ & $2(1)$ & $4(1)$ & $10(1)$ \\
\hline
\end{tabular}

${ }^{a}$ In general, the reaction was carried out in toluene with [CL] $=2.0 \mathrm{M}$ at $25^{\circ} \mathrm{C}$.

${ }^{b}$ The data were determined from ${ }^{1} \mathrm{H}$ NMR analysis. 


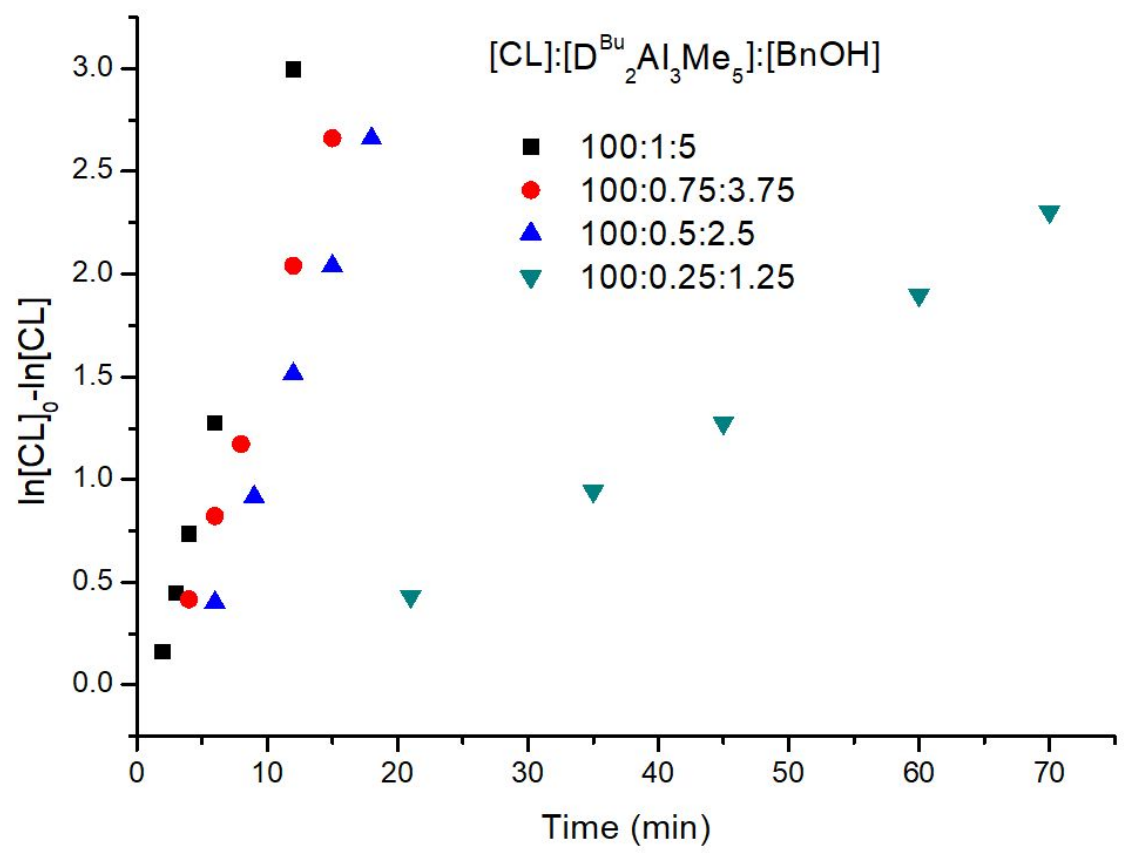

Figure S2. First-order kinetic plots of CL polymerization with various concentrations of $\left[\mathbf{D}^{\mathrm{Bu}}{ }_{2} \mathbf{A l}_{3} \mathbf{M e}_{5} \cdot 5 \mathrm{BnOH}\right]$ plotted against time with $[\mathrm{CL}]=2.0 \mathrm{M}$ in toluene $5 \mathrm{~mL}$

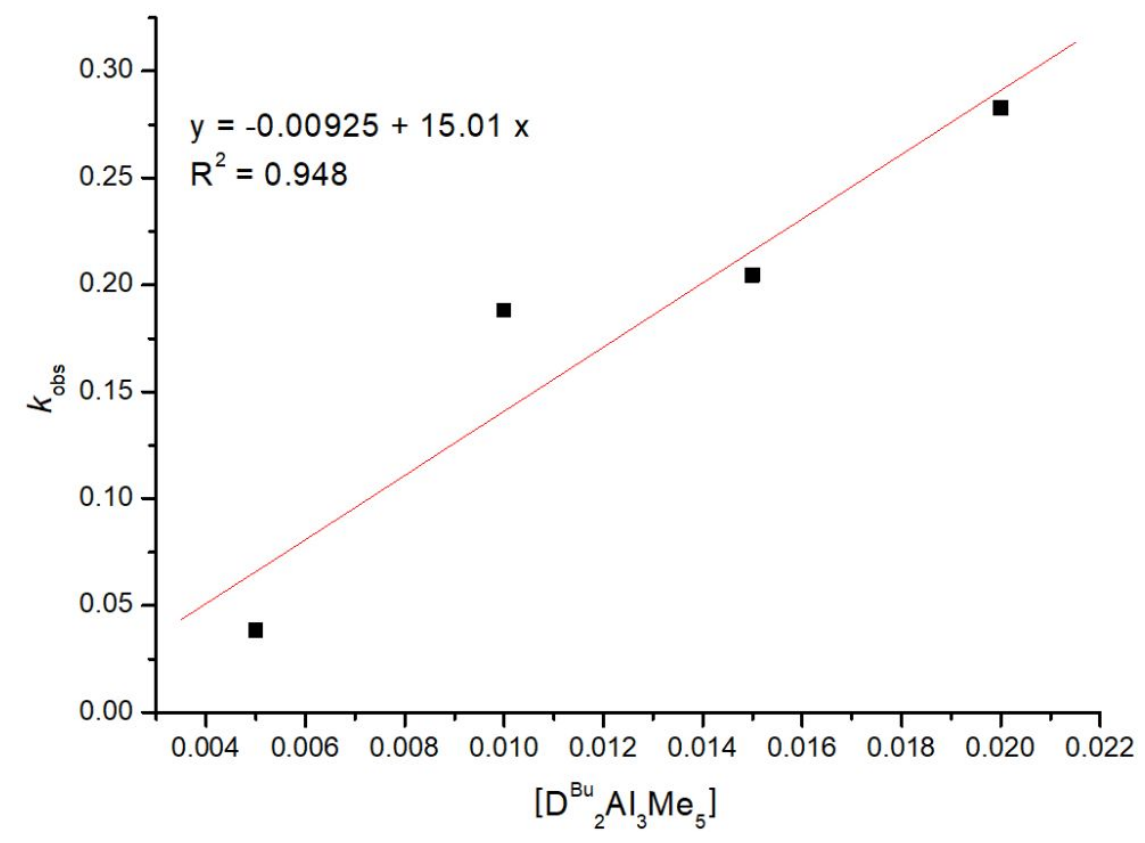

Figure S3. Linear plot of $k_{\mathrm{obs}}$ versus $\left[\mathrm{D}^{\mathrm{Bu}}{ }_{2} \mathrm{Al}_{3} \mathbf{M e}_{5} \cdot 5 \mathrm{BnOH}\right]$ for the polymerization of $\mathrm{CL}$ with $[\mathrm{CL}]=2.0 \mathrm{M}$ in toluene $(5 \mathrm{~mL})$ at room temperature. 

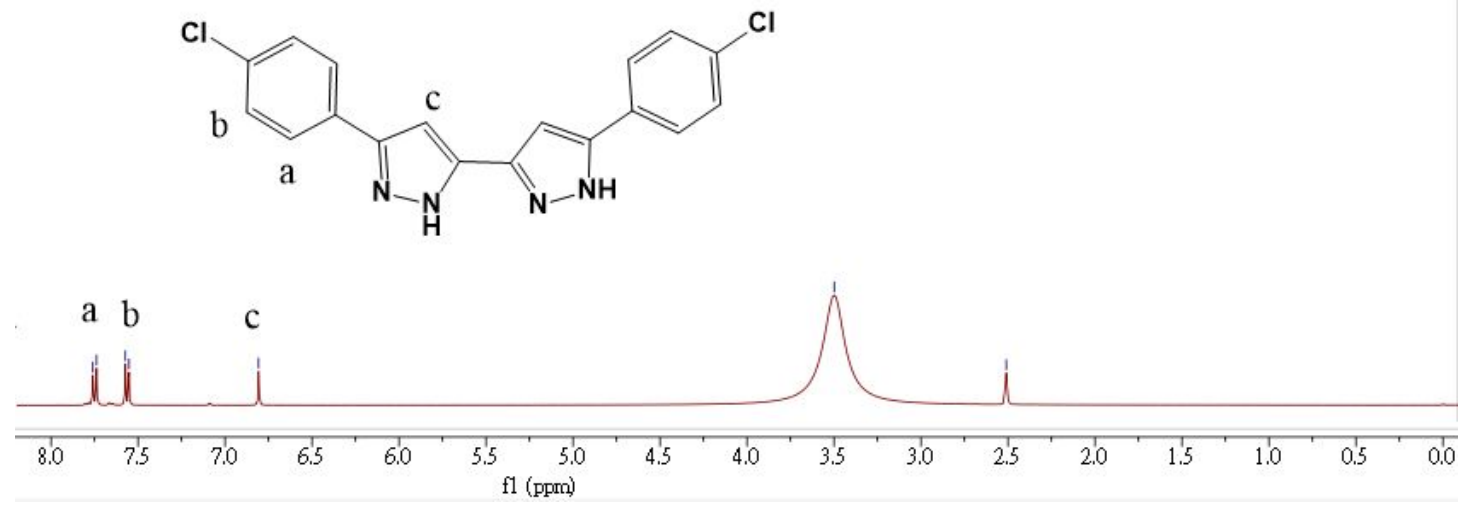

Figure S4. ${ }^{1} \mathrm{H}$ NMR spectrum of $\mathbf{D}^{\mathrm{Br}}-\mathbf{H}$ in DMSO-d 6
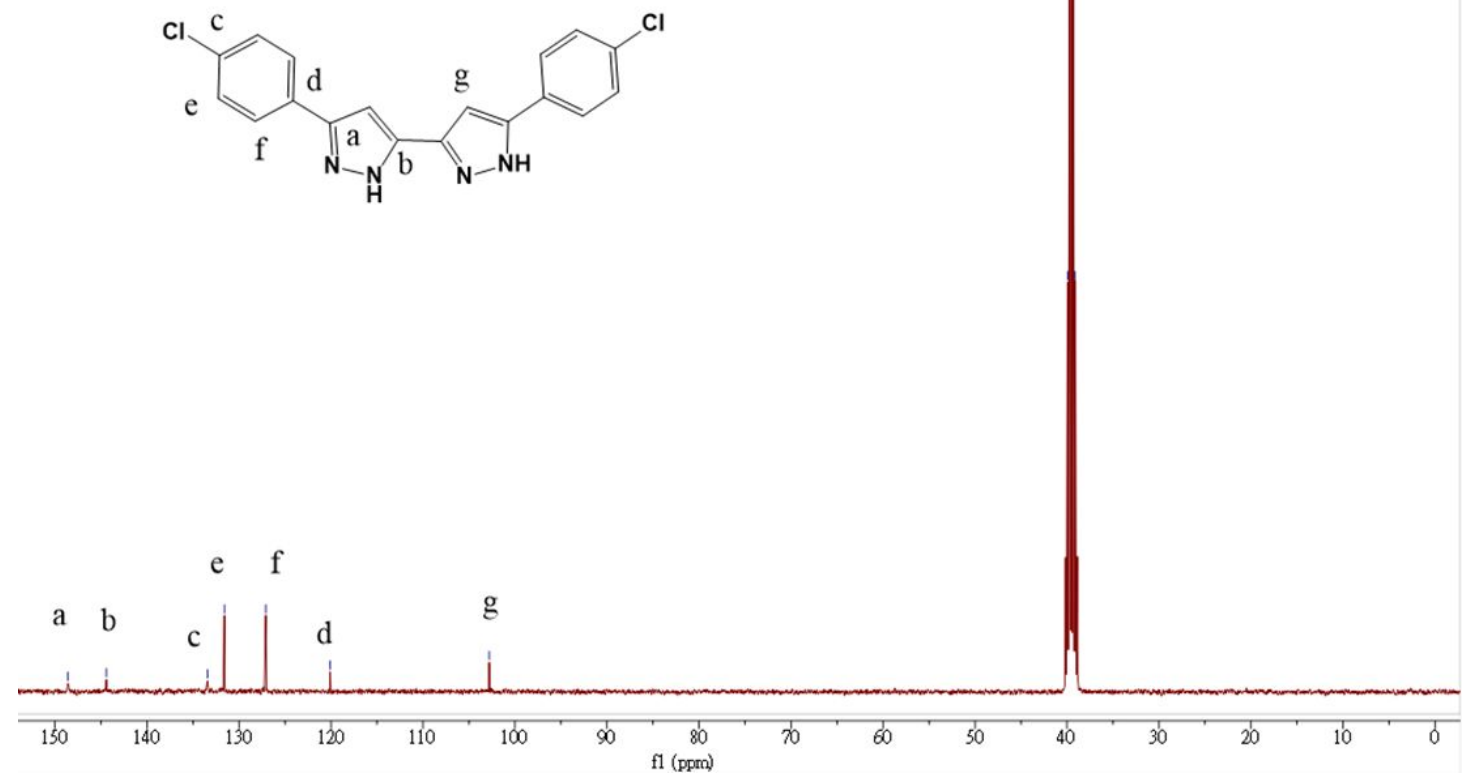

Figure S5. ${ }^{13} \mathrm{C}$ NMR spectrum of $\mathbf{D}^{\mathrm{Br}}-\mathbf{H}$ in DMSO- $\mathrm{d}_{6}$ 

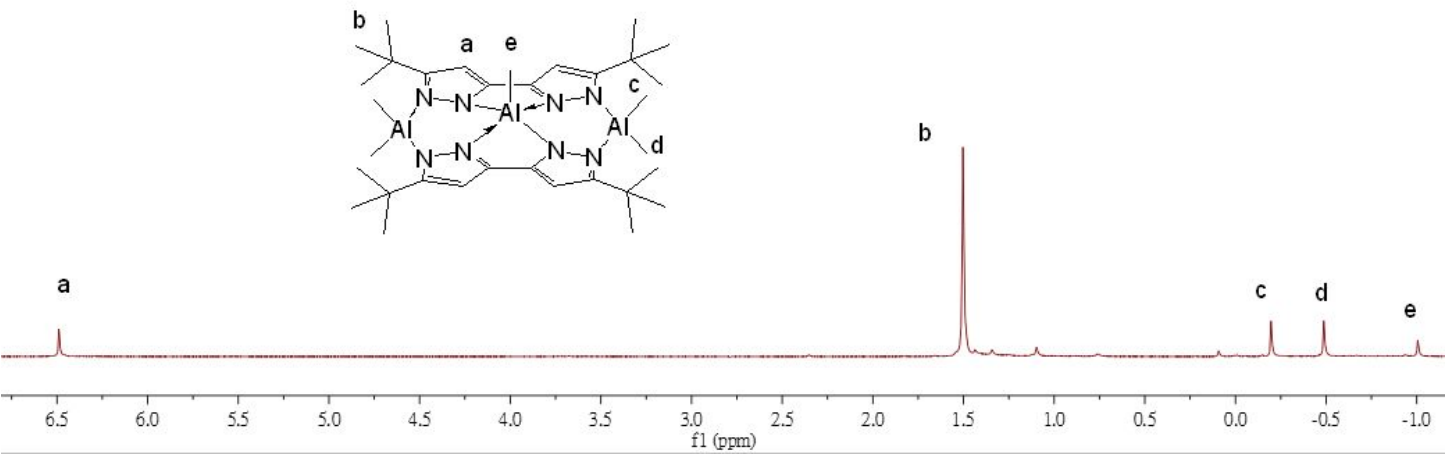

Figure S6. ${ }^{1} \mathrm{H}$ NMR spectrum of $\mathbf{D}^{\mathbf{B u}}{ }_{2} \mathbf{A l}_{3} \mathbf{M e}_{5}$ in $\mathrm{CDCl}_{3}$

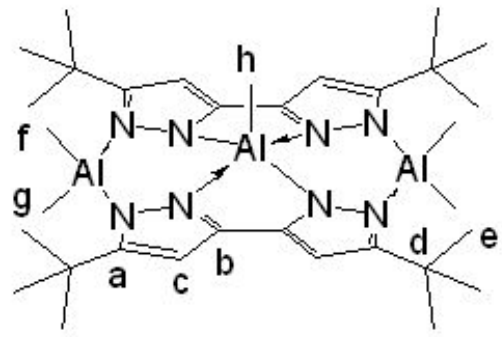

a

b

c

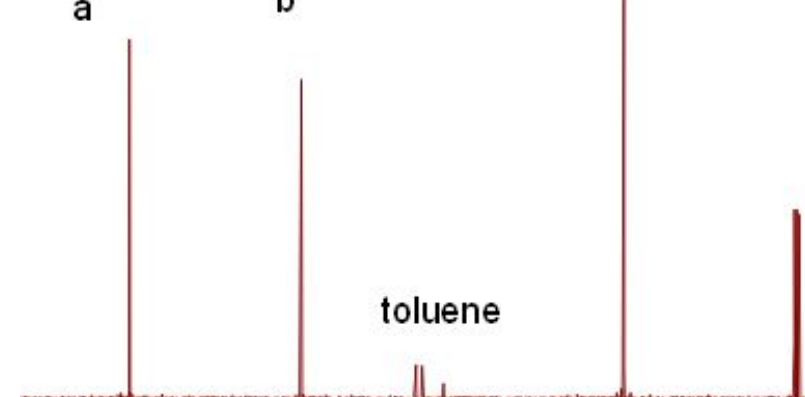

toluene $f+g+h$

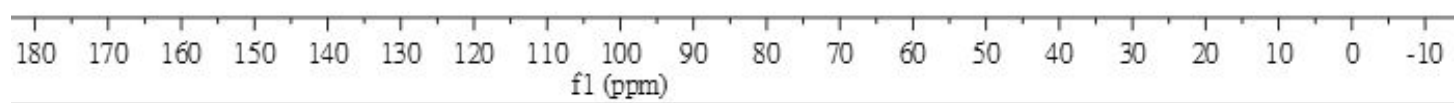

Figure S7. ${ }^{13} \mathrm{C}$ NMR spectrum of $\mathbf{D}^{\mathbf{B u}}{ }_{2} \mathbf{A l}_{3} \mathbf{M e}_{5}$ in $\mathrm{CDCl}_{3}$ 

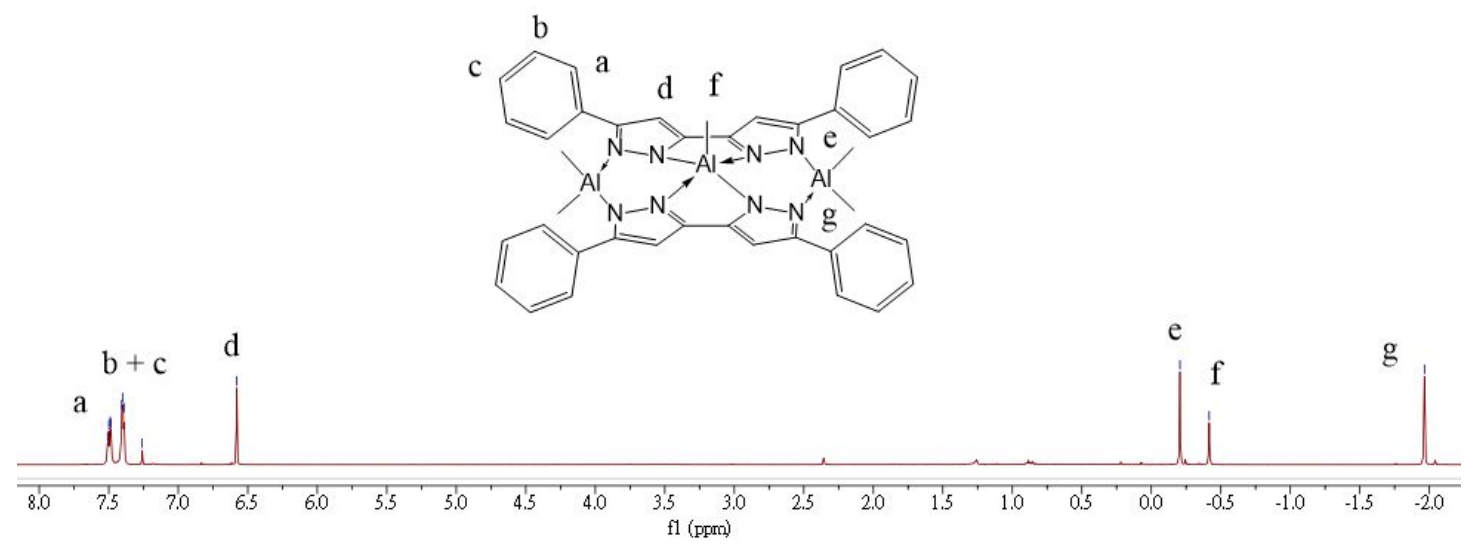

Figure S8. ${ }^{1} \mathrm{H}$ NMR spectrum of $\mathbf{D}^{\mathbf{p h}}{ }_{2} \mathbf{A l}_{3} \mathbf{M e}_{5}$ in $\mathrm{CDCl}_{3}$
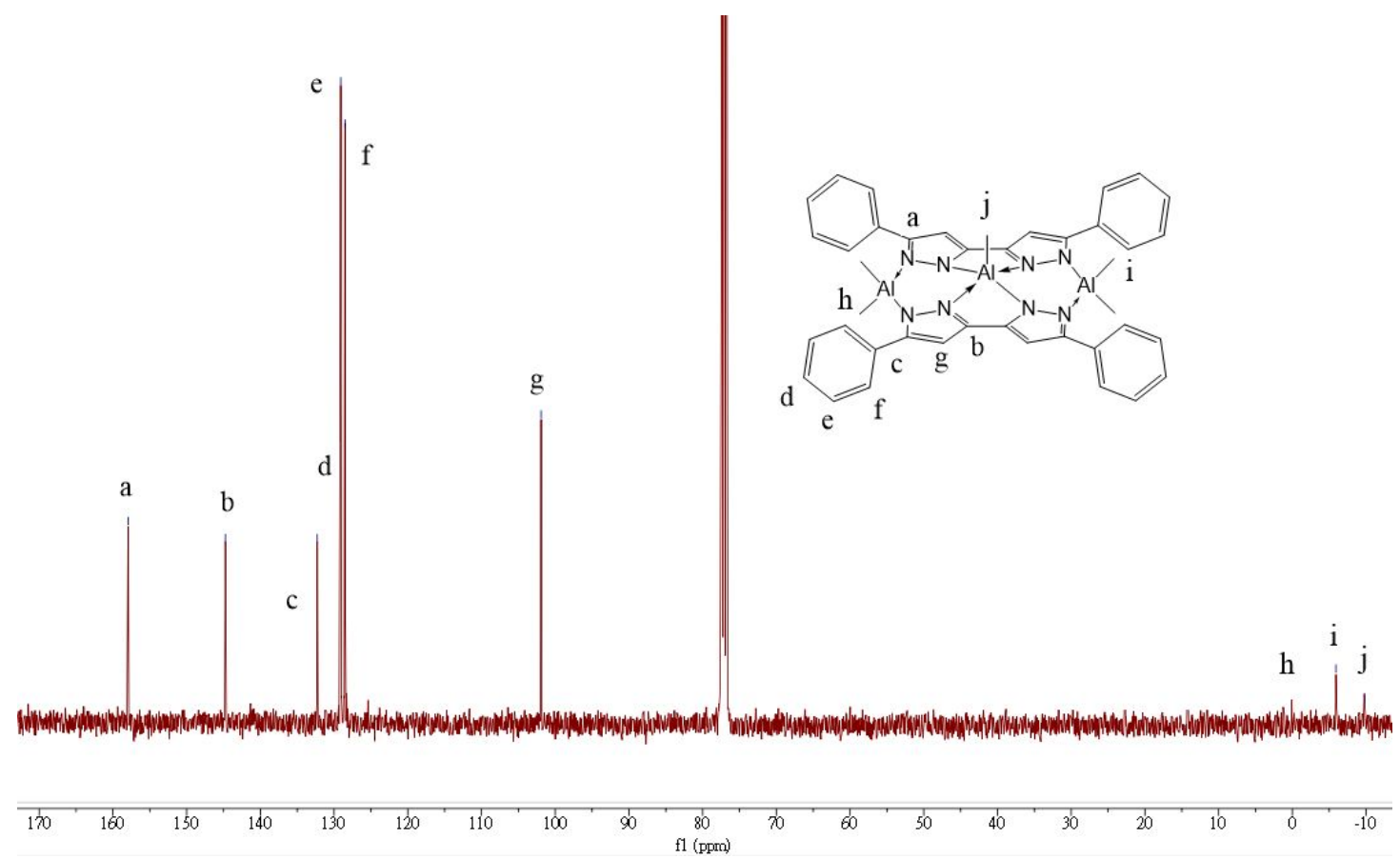

Figure S9. ${ }^{13} \mathrm{C}$ NMR spectrum of $\mathbf{D}^{\mathbf{p h}}{ }_{2} \mathbf{A l}_{3} \mathbf{M e}_{5}$ in $\mathrm{CDCl}_{3}$ 

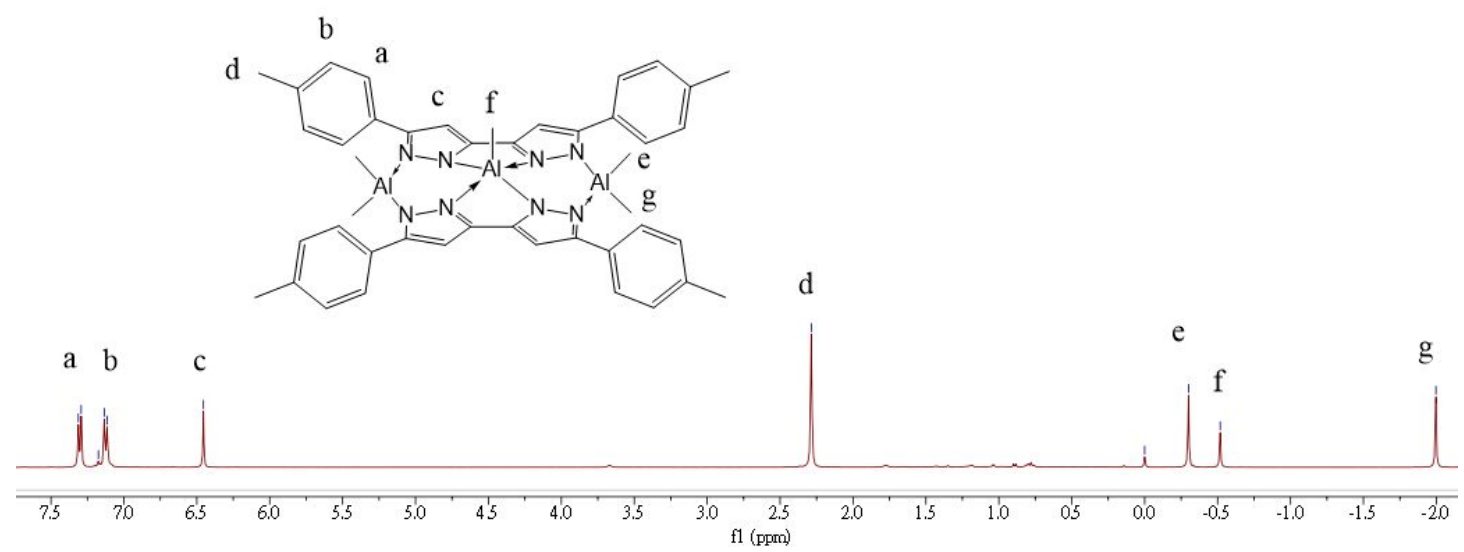

Figure S10. ${ }^{1} \mathrm{H}$ NMR spectrum of $\mathbf{D}^{\text {tolyl }}{ }_{2} \mathbf{A l}_{3} \mathbf{M e}_{5}$ in $\mathrm{CDCl}_{3}$
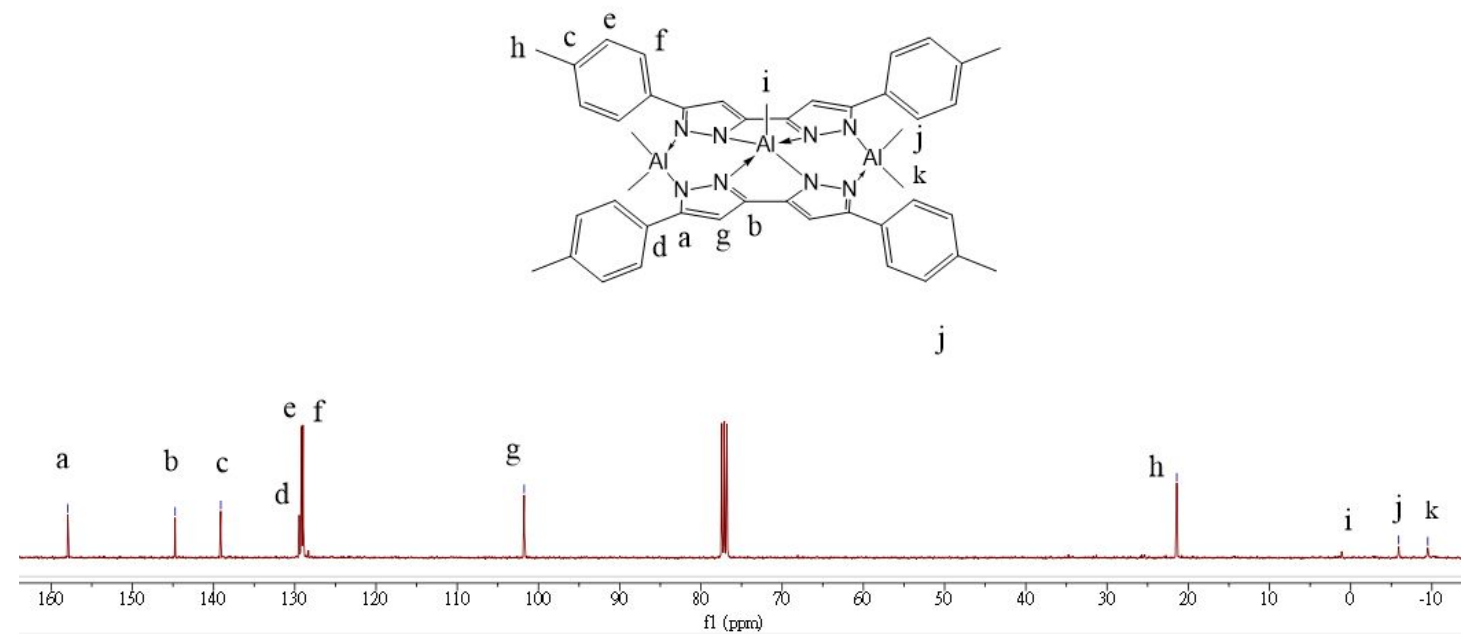

Figure S11. ${ }^{13} \mathrm{C}$ NMR spectrum of $\mathbf{D}^{\text {tolyl }}{ }_{2} \mathbf{A l}_{3} \mathbf{M e}_{5}$ in $\mathrm{CDCl}_{3}$ 


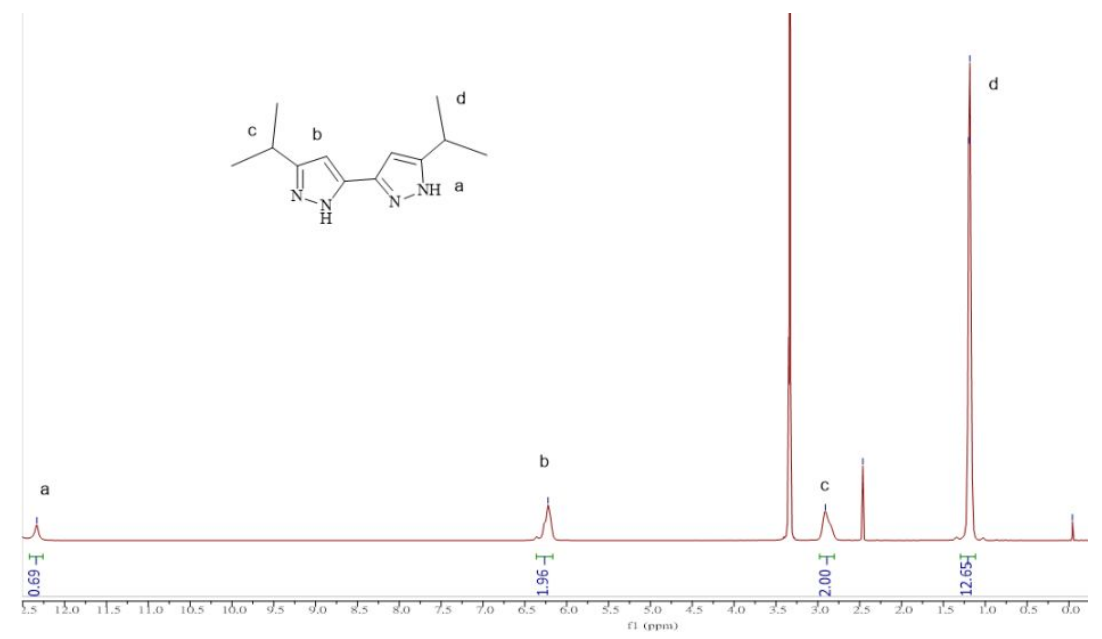

Figure S12. ${ }^{1} \mathrm{H}$ NMR spectrum of $\mathbf{D}^{\mathrm{Pr}}-\mathbf{H}$ in DMSO-d 6

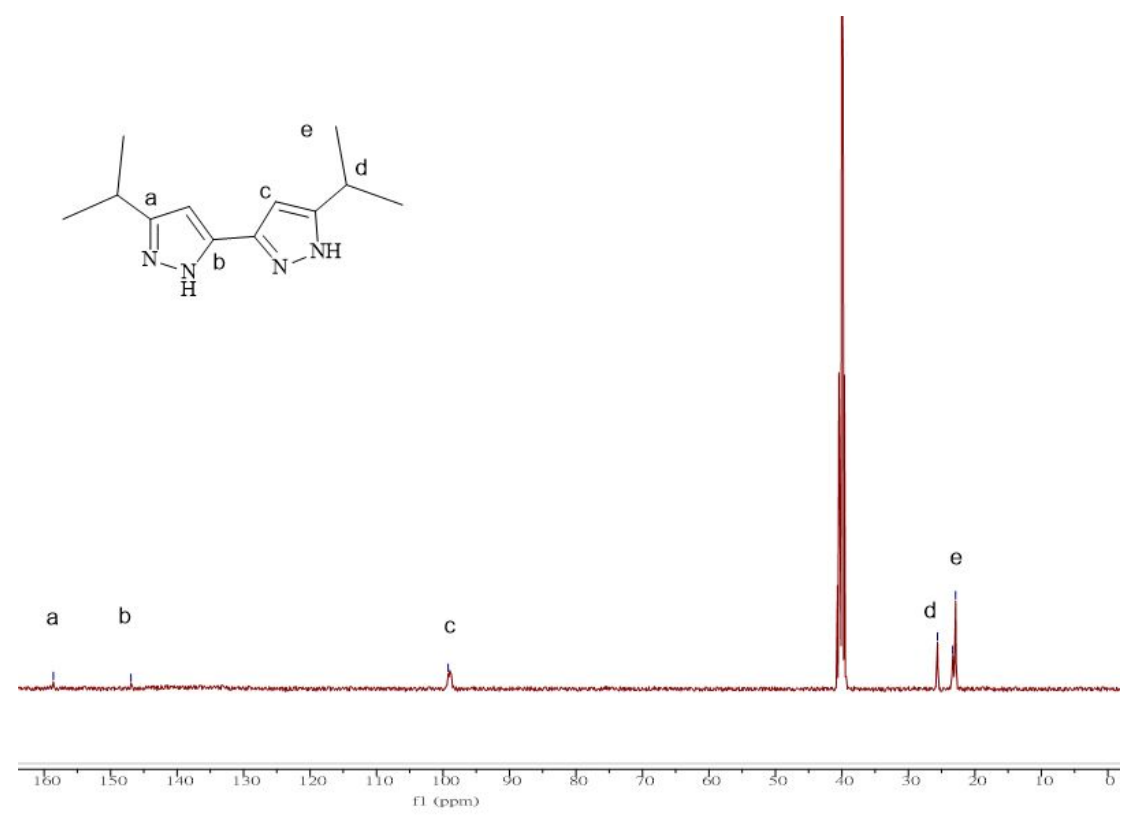

Figure S13. ${ }^{13} \mathrm{C}$ NMR spectrum of $\mathbf{D}^{\mathrm{Pr}}-\mathbf{H}$ in DMSO-d 6 

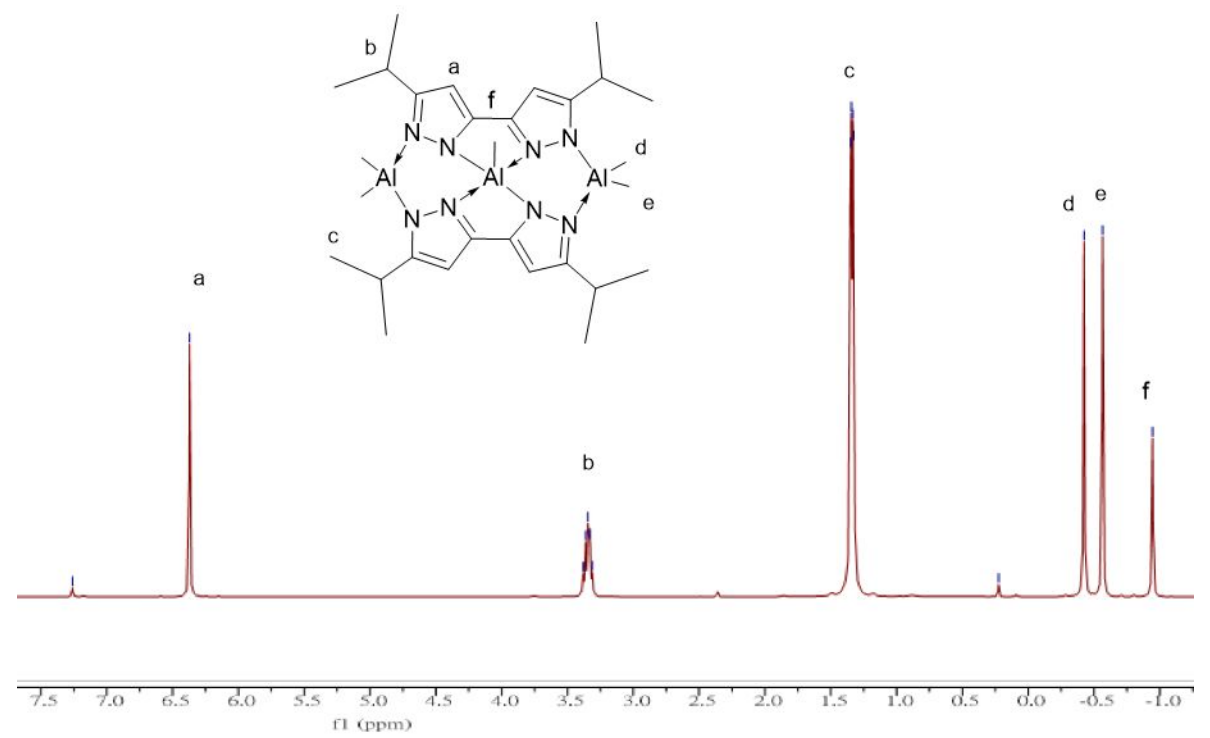

Figure S14. ${ }^{1} \mathrm{H}$ NMR spectrum of $\mathbf{D}^{\mathrm{Pr}_{2}} \mathbf{A l}_{\mathbf{3}} \mathbf{M e}_{5}$ in $\mathrm{CDCl}_{3}$
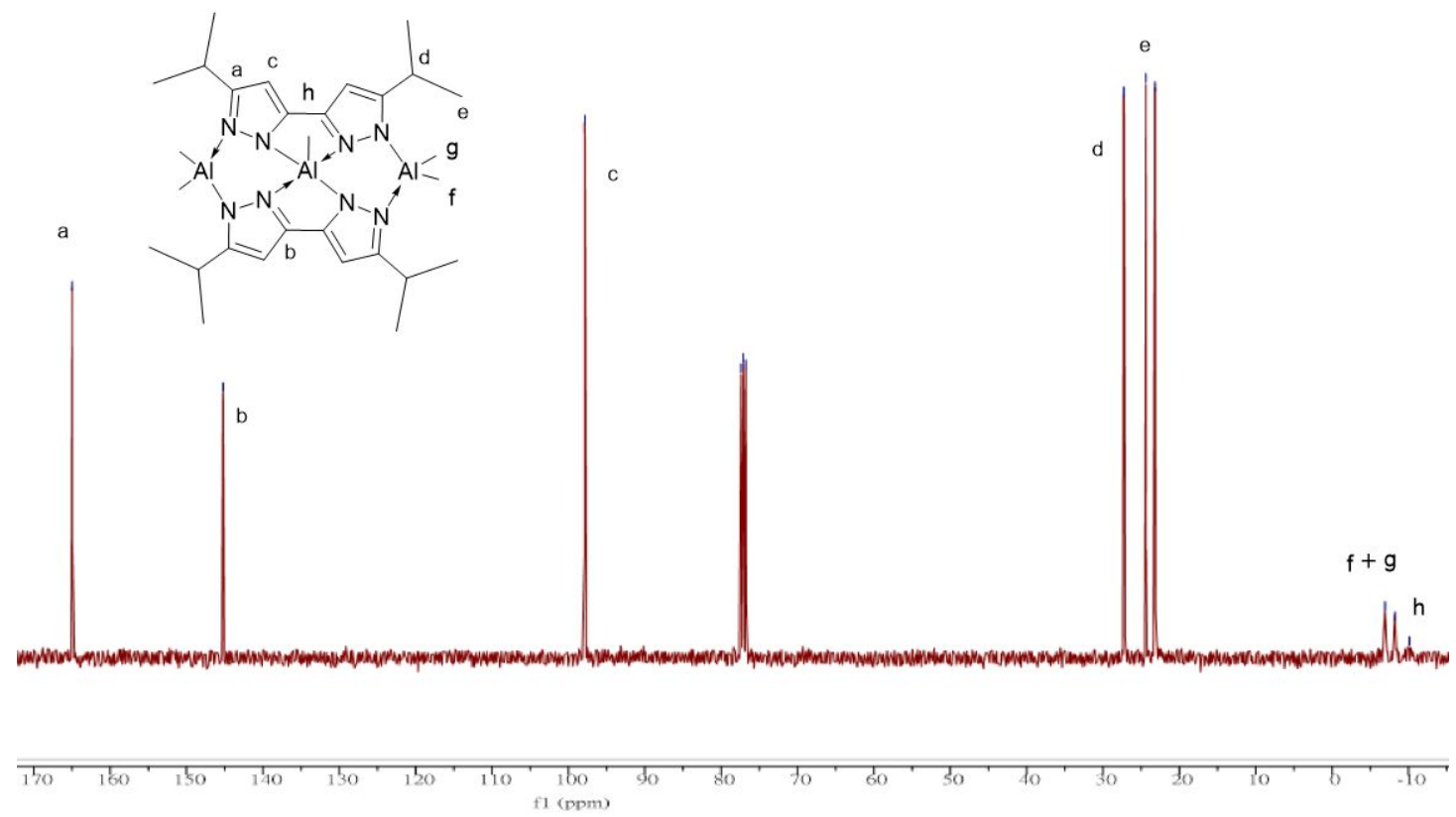

Figure S15. ${ }^{13} \mathrm{C}$ NMR spectrum of $\mathbf{D}^{\mathrm{Pr}_{2}} \mathrm{Al}_{3} \mathbf{M e}_{5}$ in $\mathrm{CDCl}_{3}$ 


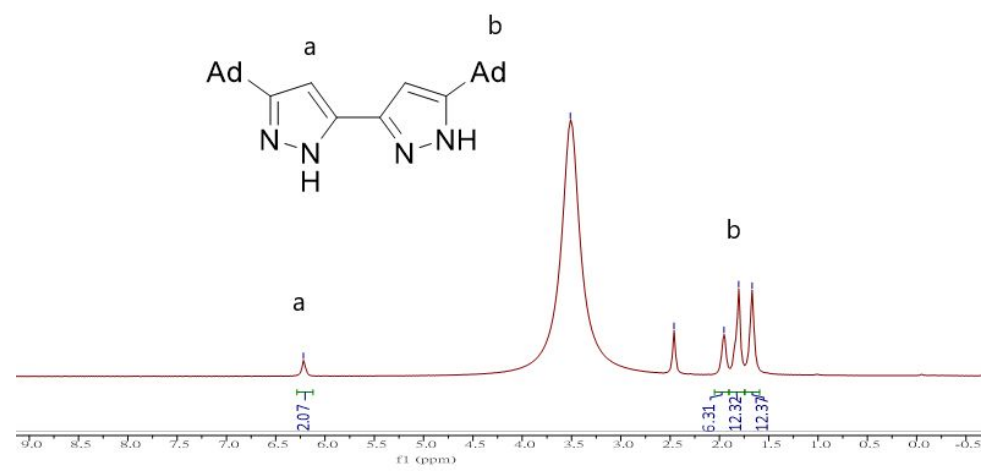

Figure S16. ${ }^{1} \mathrm{H}$ NMR spectrum of $\mathbf{D}^{\mathbf{A d}}-\mathbf{H}$ in DMSO-d $\mathrm{d}_{6}$

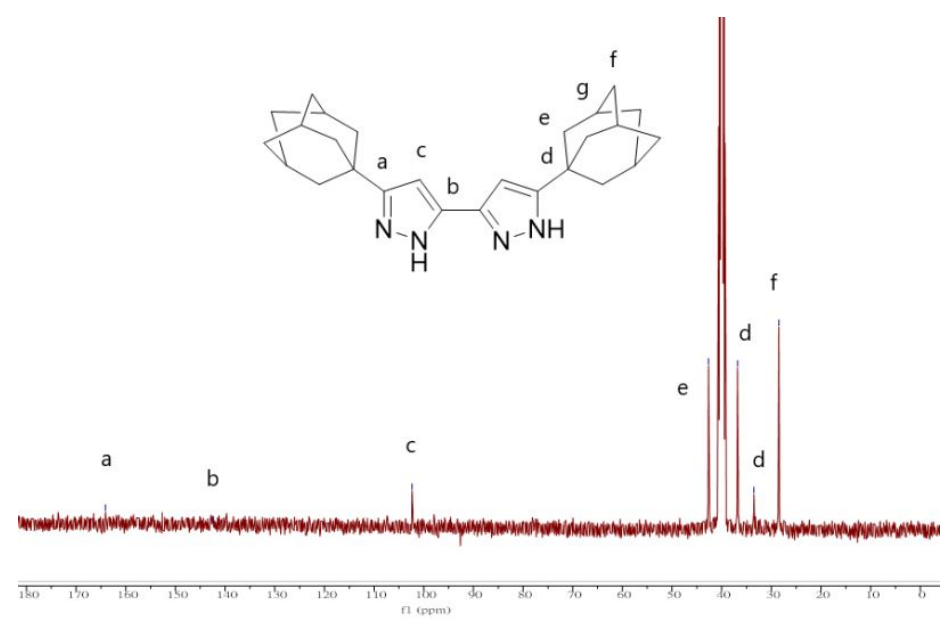

Figure S17. ${ }^{13} \mathrm{C}$ NMR spectrum of $\mathbf{D}^{\mathbf{A d}}-\mathbf{H}$ in DMSO-d 6 


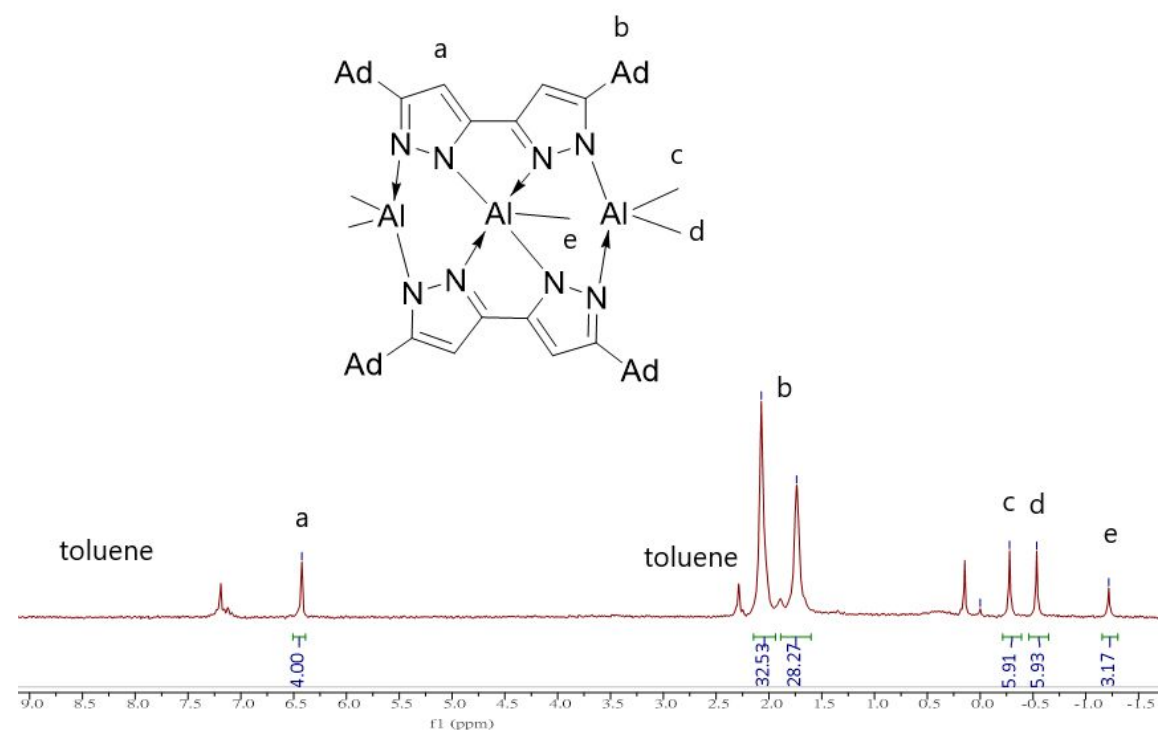

Figure S18. ${ }^{1} \mathrm{H}$ NMR spectrum of $\mathbf{D}^{\mathbf{A d}_{2}} \mathbf{A l}_{3} \mathbf{M e}_{5}$ in $\mathrm{CDCl}_{3}$

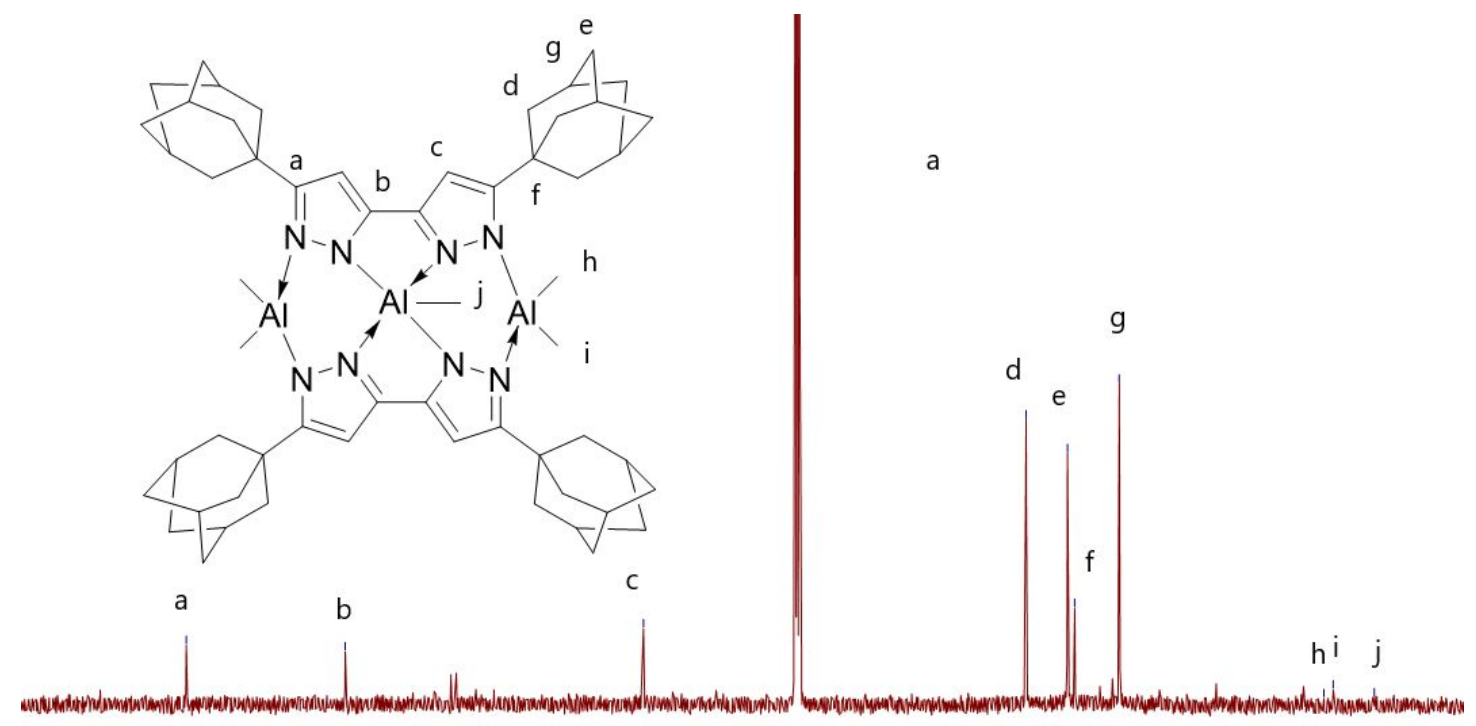

Figure S19. ${ }^{13} \mathrm{C}$ NMR spectrum of $\mathbf{D}^{\mathbf{A d}}{ }_{2} \mathbf{A l}_{3} \mathbf{M e}_{5}$ in $\mathrm{CDCl}_{3}$ 
$\mathbf{L}^{\mathrm{Bu}}{ }_{2} \mathrm{Al}_{2} \mathbf{M e}_{4}$

Table S3. Atomic coordinates of $\mathrm{L}^{\mathrm{Bu}}{ }_{2} \mathrm{Al}_{2} \mathrm{Me}_{4}$

$\begin{array}{lcrcc}\text { Center } & \text { Atomic } & \text { Atomic } & \text { Coordinates (Angstroms) } \\ \text { Number } & \text { Number } & \text { Type } & X & Y \\ Z & & & \end{array}$

\begin{tabular}{|c|c|c|c|c|c|}
\hline 1 & 13 & 0 & -0.006934 & -1.762329 & -0.016940 \\
\hline 2 & 7 & 0 & -1.417924 & -0.483258 & -0.507444 \\
\hline 3 & 7 & 0 & 1.425104 & -0.509503 & 0.480372 \\
\hline 4 & 6 & 0 & -2.706938 & -0.745293 & -0.828391 \\
\hline 5 & 6 & 0 & -3.539900 & 0.000068 & 0.000424 \\
\hline 6 & 1 & 0 & -4.469900 & 0.000086 & 0.000451 \\
\hline 7 & 6 & 0 & -3.101987 & -1.692201 & -1.951510 \\
\hline 8 & 6 & 0 & -4.572993 & -1.450608 & -2.325701 \\
\hline 9 & 1 & 0 & -4.686984 & -0.540087 & -2.607593 \\
\hline 10 & 1 & 0 & -5.129975 & -1.621491 & -1.562620 \\
\hline 11 & 1 & 0 & -4.821025 & -2.040325 & -3.040122 \\
\hline 12 & 6 & 0 & -2.943003 & -3.152846 & -1.511756 \\
\hline 13 & 1 & 0 & -3.498985 & -3.319967 & -0.746258 \\
\hline 14 & 1 & 0 & -2.025000 & -3.320449 & -1.282446 \\
\hline 15 & 1 & 0 & -3.205035 & -3.734179 & -2.229295 \\
\hline 16 & 6 & 0 & -2.226017 & -1.424497 & -3.182912 \\
\hline 17 & 1 & 0 & -2.335007 & -0.512203 & -3.462601 \\
\hline 18 & 1 & 0 & -2.488048 & -2.011446 & -3.895021 \\
\hline 19 & 1 & 0 & -1.306014 & -1.583287 & -2.960696 \\
\hline 20 & 6 & 0 & 2.711107 & -0.794607 & 0.782482 \\
\hline 21 & 6 & 0 & 3.539100 & -0.000068 & 0.000225 \\
\hline 22 & 1 & 0 & 4.468100 & -0.000086 & 0.000199 \\
\hline 23 & 6 & 0 & 3.109118 & -1.794873 & 1.858320 \\
\hline 24 & 6 & 0 & 2.283158 & -1.526884 & 3.121404 \\
\hline 25 & 1 & 0 & 2.425184 & -0.622762 & 3.411122 \\
\hline 26 & 1 & 0 & 2.555166 & -2.131885 & 3.815467 \\
\hline 27 & 1 & 0 & 1.353150 & -1.658989 & 2.929138 \\
\hline 28 & 6 & 0 & 2.898077 & -3.240353 & 1.384432 \\
\hline 29 & 1 & 0 & 3.417051 & -3.397213 & 0.592003 \\
\hline 30 & 1 & 0 & 1.968068 & -3.381406 & 1.191199 \\
\hline
\end{tabular}




\begin{tabular}{|c|c|c|c|c|c|}
\hline 31 & 1 & 0 & 3.176084 & -3.846806 & 2.073309 \\
\hline 32 & 6 & 0 & 4.594130 & -1.623474 & 2.189593 \\
\hline 33 & 1 & 0 & 4.754156 & -0.725397 & 2.488715 \\
\hline 34 & 1 & 0 & 5.119105 & -1.797422 & 1.406383 \\
\hline 35 & 1 & 0 & 4.840138 & -2.239626 & 2.884465 \\
\hline 36 & 6 & 0 & -0.824910 & -2.712717 & 1.500837 \\
\hline 37 & 1 & 0 & -1.508930 & -3.303417 & 1.177582 \\
\hline 38 & 1 & 0 & -1.210881 & -2.076169 & 2.107679 \\
\hline 39 & 1 & 0 & -0.152907 & -3.223821 & 1.958603 \\
\hline 40 & 6 & 0 & 0.788004 & -2.716250 & -1.541159 \\
\hline 41 & 1 & 0 & 1.126999 & -2.080486 & -2.175592 \\
\hline 42 & 1 & 0 & 0.119982 & -3.262413 & -1.960187 \\
\hline 43 & 1 & 0 & 1.506002 & -3.271766 & -1.231014 \\
\hline 44 & 7 & 0 & -1.417876 & 0.483419 & 0.507179 \\
\hline 45 & 6 & 0 & -2.706862 & 0.744510 & 0.828092 \\
\hline 46 & 6 & 0 & -3.101813 & 1.692320 & 1.952334 \\
\hline 47 & 6 & 0 & -4.572807 & 1.450891 & 2.325614 \\
\hline 48 & 1 & 0 & -4.686816 & 0.540374 & 2.607512 \\
\hline 49 & 1 & 0 & -5.129825 & 1.621795 & 1.562565 \\
\hline 50 & 1 & 0 & -4.820775 & 2.039515 & 3.040935 \\
\hline 51 & 6 & 0 & -2.942797 & 3.153066 & 1.511578 \\
\hline 52 & 1 & 0 & -3.498815 & 3.320101 & 0.747105 \\
\hline 53 & 1 & 0 & -2.024800 & 3.320526 & 1.283209 \\
\hline 54 & 1 & 0 & -3.204765 & 3.734410 & 2.229131 \\
\hline 55 & 6 & 0 & -2.225783 & 1.424690 & 3.182693 \\
\hline 56 & 1 & 0 & -2.334793 & 0.512399 & 3.462388 \\
\hline 57 & 1 & 0 & -2.487752 & 2.011541 & 3.895811 \\
\hline 58 & 1 & 0 & -1.305786 & 1.583444 & 2.960425 \\
\hline 59 & 7 & 0 & 1.425096 & 0.509448 & -0.479803 \\
\hline 60 & 6 & 0 & 2.711093 & 0.794502 & -0.781984 \\
\hline 61 & 6 & 0 & 3.109082 & 1.794860 & -1.858839 \\
\hline 62 & 6 & 0 & 2.283042 & 1.526796 & -3.120883 \\
\hline 63 & 1 & 0 & 2.425016 & 0.622775 & -3.411602 \\
\hline 64 & 1 & 0 & 2.555034 & 2.131786 & -3.814961 \\
\hline 65 & 1 & 0 & 1.353050 & 1.658937 & -2.928564 \\
\hline 66 & 6 & 0 & 2.898123 & 3.240349 & -1.384939 \\
\hline 67 & 1 & 0 & 3.417149 & 3.397081 & -0.591546 \\
\hline 68 & 1 & 0 & 1.968132 & 3.381437 & -1.191654 \\
\hline
\end{tabular}




$\begin{array}{rrrrrr}69 & 1 & 0 & 3.176116 & 3.846791 & -2.073832 \\ 70 & 6 & 0 & 4.594070 & 1.623404 & -2.190196 \\ 71 & 1 & 0 & 4.754044 & 0.725213 & -2.488333 \\ 72 & 1 & 0 & 5.119095 & 1.797225 & -1.406021 \\ 73 & 1 & 0 & 4.840062 & 2.239439 & -2.884087 \\ 74 & 13 & 0 & -0.006866 & 1.762436 & 0.016596 \\ 75 & 6 & 0 & -0.824890 & 2.712856 & -1.501135 \\ 76 & 1 & 0 & -1.508870 & 3.302480 & -1.176955 \\ 77 & 1 & 0 & -1.210919 & 2.076215 & -2.106961 \\ 78 & 1 & 0 & -0.152893 & 3.223934 & -1.958939 \\ 79 & 6 & 0 & 0.788196 & 2.716327 & 1.540771 \\ 80 & 1 & 0 & 1.127201 & 2.079448 & 2.176071 \\ 81 & 1 & 0 & 0.120218 & 3.262408 & 1.960830 \\ 82 & 1 & 0 & 1.506198 & 3.271815 & 1.230585\end{array}$

Table S4. Mulliken charge of $\mathrm{L}^{\mathrm{Bu}_{2}} \mathrm{Al}_{2} \mathrm{Me}_{4}$

$\begin{array}{rlc} & & 1 \\ 1 & \mathrm{Al} & 0.851737 \\ 2 & \mathrm{~N} & -0.470490 \\ 3 & \mathrm{~N} & -0.467601 \\ 4 & \mathrm{C} & 0.360374 \\ 5 & \mathrm{C} & -0.258982 \\ 6 & \mathrm{H} & 0.092915 \\ 7 & \mathrm{C} & -0.081625 \\ 8 & \mathrm{C} & -0.443937 \\ 9 & \mathrm{H} & 0.159540 \\ 10 & \mathrm{H} & 0.156063 \\ 11 & \mathrm{H} & 0.153572 \\ 12 & \mathrm{C} & -0.450616 \\ 13 & \mathrm{H} & 0.161032 \\ 14 & \mathrm{H} & 0.191031 \\ 15 & \mathrm{H} & 0.153032 \\ 16 & \mathrm{C} & -0.448514 \\ 17 & \mathrm{H} & 0.161042 \\ 18 & \mathrm{H} & 0.146029 \\ 19 & \mathrm{H} & 0.190034 \\ 20 & \mathrm{C} & 0.361626\end{array}$




\begin{tabular}{|c|c|c|}
\hline & $\mathrm{C}$ & 761 \\
\hline & $\mathrm{H}$ & 0.090941 \\
\hline & $\mathrm{C}$ & -0.081782 \\
\hline & $\mathrm{C}$ & -0.447490 \\
\hline & $\mathrm{H}$ & 0.160706 \\
\hline & $\mathrm{H}$ & 0.145981 \\
\hline & $\mathrm{H}$ & 0.190296 \\
\hline & $\mathrm{C}$ & -0.451806 \\
\hline & $\mathrm{H}$ & 0.162460 \\
\hline & $\mathrm{H}$ & 0.190662 \\
\hline & $\mathrm{H}$ & 0.152970 \\
\hline & $\mathrm{C}$ & -0.442666 \\
\hline & $\mathrm{H}$ & 0.158514 \\
\hline & $\mathrm{H}$ & 0.156326 \\
\hline & $\mathrm{H}$ & 0.153460 \\
\hline & $\mathrm{C}$ & -0.757472 \\
\hline & $\mathrm{H}$ & 0.159081 \\
\hline & $\mathrm{H}$ & 0.166747 \\
\hline & $\mathrm{H}$ & 0.151092 \\
\hline & $\mathrm{C}$ & -0.757557 \\
\hline & $\mathrm{H}$ & 0.166863 \\
\hline & $\mathrm{H}$ & 0.151471 \\
\hline & $\mathrm{H}$ & 0.159131 \\
\hline & $\mathrm{N}$ & -0.470432 \\
\hline & $\mathrm{C}$ & 0.360363 \\
\hline & $\mathrm{C}$ & -0.081321 \\
\hline & $\mathrm{C}$ & -0.444007 \\
\hline & $\mathrm{H}$ & 0.159499 \\
\hline & $\mathrm{H}$ & 0.156080 \\
\hline & $\mathrm{H}$ & 0.153562 \\
\hline & $\mathrm{C}$ & -0.450495 \\
\hline & $\mathrm{H}$ & 0.161059 \\
\hline & $\mathrm{H}$ & 0.190976 \\
\hline & $\mathrm{H}$ & 0.152953 \\
\hline & $\mathrm{C}$ & -0.448602 \\
\hline & $\mathrm{H}$ & 0.161052 \\
\hline & $\mathrm{H}$ & 0.145992 \\
\hline & $\mathrm{H}$ & 0.19006 \\
\hline
\end{tabular}




$\begin{array}{ccc}59 & \mathrm{~N} & -0.467510 \\ 60 & \mathrm{C} & 0.361391 \\ 61 & \mathrm{C} & -0.081618 \\ 62 & \mathrm{C} & -0.447429 \\ 63 & \mathrm{H} & 0.160686 \\ 64 & \mathrm{H} & 0.145969 \\ 65 & \mathrm{H} & 0.190346 \\ 66 & \mathrm{C} & -0.451930 \\ 67 & \mathrm{H} & 0.162419 \\ 68 & \mathrm{H} & 0.190678 \\ 69 & \mathrm{H} & 0.152997 \\ 70 & \mathrm{C} & -0.442554 \\ 71 & \mathrm{H} & 0.158534 \\ 72 & \mathrm{H} & 0.156212 \\ 73 & \mathrm{H} & 0.153528 \\ 74 & \mathrm{Al} & 0.851725 \\ 75 & \mathrm{C} & -0.757490 \\ 76 & \mathrm{H} & 0.159173 \\ 77 & \mathrm{H} & 0.166827 \\ 78 & \mathrm{H} & 0.151090 \\ 79 & \mathrm{C} & -0.757569 \\ 80 & \mathrm{H} & 0.166690 \\ 81 & \mathrm{H} & 0.151470 \\ 82 & \mathrm{H} & 0.159191\end{array}$


$\mathrm{D}^{\mathrm{Bu}}{ }_{2} \mathrm{Al}_{3} \mathrm{Me}_{5}$

Table S5. Atomic coordinates of $\mathrm{D}^{\mathrm{Bu}}{ }_{2} \mathrm{Al}_{3} \mathrm{Me}_{5}$

$\begin{array}{lcccc}\text { Center } & \text { Atomic } & \text { Atomic } & \text { Coordinates (Angstroms) } \\ \text { Number } & \text { Number } & \text { Type } & X & Y \\ Z & & & \end{array}$

\begin{tabular}{|c|c|c|c|c|c|}
\hline 1 & 6 & 0 & -4.306992 & 0.077070 & -2.633331 \\
\hline 2 & 1 & 0 & -3.934641 & 0.863749 & -3.082931 \\
\hline 3 & 1 & 0 & -5.284831 & 0.110872 & -2.670759 \\
\hline 4 & 1 & 0 & -3.990834 & -0.736089 & -3.082242 \\
\hline 5 & 6 & 0 & -4.959765 & 0.066904 & 0.772277 \\
\hline 6 & 1 & 0 & -5.884375 & 0.062834 & 0.447973 \\
\hline 7 & 1 & 0 & -4.806045 & 0.870756 & 1.311218 \\
\hline 8 & 1 & 0 & -4.804087 & -0.729340 & 1.320591 \\
\hline 9 & 6 & 0 & -0.199746 & 0.196369 & -3.059226 \\
\hline 10 & 6 & 0 & 5.661293 & 0.010769 & -1.109307 \\
\hline 11 & 1 & 0 & 6.020220 & -0.826055 & -1.471749 \\
\hline 12 & 1 & 0 & 5.891874 & 0.082634 & -0.160257 \\
\hline 13 & 1 & 0 & 6.046513 & 0.768887 & -1.595618 \\
\hline 14 & 6 & 0 & 3.242383 & 0.058011 & -3.216507 \\
\hline 15 & 1 & 0 & 2.676490 & -0.712458 & -3.430937 \\
\hline 16 & 1 & 0 & 4.063138 & 0.016740 & -3.749279 \\
\hline 17 & 1 & 0 & 2.758597 & 0.884751 & -3.423410 \\
\hline 18 & 6 & 0 & -2.822452 & -2.864293 & -0.346920 \\
\hline 19 & 6 & 0 & -4.183053 & -3.535094 & -0.272667 \\
\hline 20 & 6 & 0 & -5.239591 & -2.853874 & -1.142272 \\
\hline 21 & 1 & 0 & -6.068691 & -3.376406 & -1.119376 \\
\hline 22 & 1 & 0 & -4.915964 & -2.796006 & -2.065653 \\
\hline 23 & 1 & 0 & -5.414134 & -1.951685 & -0.800692 \\
\hline 24 & 6 & 0 & -4.626671 & -3.587932 & 1.194378 \\
\hline 25 & 1 & 0 & -3.970567 & -4.094241 & 1.716749 \\
\hline 26 & 1 & 0 & -5.502514 & -4.024918 & 1.255768 \\
\hline 27 & 1 & 0 & -4.692042 & -2.675759 & 1.548866 \\
\hline 28 & 6 & 0 & -4.044756 & -4.980853 & -0.774411 \\
\hline 29 & 1 & 0 & -3.419858 & -5.468937 & -0.199144 \\
\hline 30 & 1 & 0 & -3.705020 & -4.976258 & -1.693603 \\
\hline
\end{tabular}




\begin{tabular}{|c|c|c|c|c|c|}
\hline 31 & 1 & 0 & -4.921670 & -5.420063 & -0.751981 \\
\hline 32 & 6 & 0 & -1.612209 & -3.526824 & -0.179367 \\
\hline 33 & 1 & 0 & -1.480020 & -4.452440 & -0.005615 \\
\hline 34 & 6 & 0 & -0.646543 & -2.559828 & -0.317942 \\
\hline 35 & 6 & 0 & 0.802354 & -2.534605 & -0.286870 \\
\hline 36 & 6 & 0 & 1.779855 & -3.431682 & 0.100469 \\
\hline 37 & 1 & 0 & 1.657314 & -4.321885 & 0.410102 \\
\hline 38 & 6 & 0 & 2.974259 & -2.756502 & -0.059045 \\
\hline 39 & 6 & 0 & 4.355241 & -3.313909 & 0.232259 \\
\hline 40 & 6 & 0 & 4.226614 & -4.723245 & 0.815587 \\
\hline 41 & 1 & 0 & 3.706452 & -4.688496 & 1.644729 \\
\hline 42 & 1 & 0 & 5.118300 & -5.081008 & 1.005500 \\
\hline 43 & 1 & 0 & 3.773497 & -5.305198 & 0.170306 \\
\hline 44 & 6 & 0 & 5.149124 & -3.431561 & -1.072102 \\
\hline 45 & 1 & 0 & 4.723045 & -4.091921 & -1.656874 \\
\hline 46 & 1 & 0 & 6.065048 & -3.716232 & -0.872808 \\
\hline 47 & 1 & 0 & 5.168278 & -2.560500 & -1.522563 \\
\hline 48 & 6 & 0 & 5.061828 & -2.436963 & 1.270669 \\
\hline 49 & 1 & 0 & 5.082224 & -1.509174 & 0.953844 \\
\hline 50 & 1 & 0 & 5.977226 & -2.759094 & 1.400966 \\
\hline 51 & 1 & 0 & 4.576561 & -2.480919 & 2.120594 \\
\hline 52 & 6 & 0 & 3.023506 & 2.741058 & 0.094662 \\
\hline 53 & 6 & 0 & 4.413152 & 3.300158 & 0.333892 \\
\hline 54 & 6 & 0 & 5.149310 & 2.435994 & 1.367149 \\
\hline 55 & 1 & 0 & 4.697686 & 2.509866 & 2.234474 \\
\hline 56 & 1 & 0 & 6.075209 & 2.745064 & 1.452599 \\
\hline 57 & 1 & 0 & 5.142919 & 1.501136 & 1.074861 \\
\hline 58 & 6 & 0 & 5.173382 & 3.403816 & -0.991523 \\
\hline 59 & 1 & 0 & 5.187474 & 2.528265 & -1.431189 \\
\hline 60 & 1 & 0 & 6.093680 & 3.695844 & -0.818397 \\
\hline 61 & 1 & 0 & 4.728465 & 4.054849 & -1.573700 \\
\hline 62 & 6 & 0 & 4.295560 & 4.725151 & 0.899394 \\
\hline 63 & 1 & 0 & 3.813933 & 5.292213 & 0.260444 \\
\hline 64 & 1 & 0 & 5.193028 & 5.090842 & 1.051745 \\
\hline 65 & 1 & 0 & 3.805874 & 4.700172 & 1.748152 \\
\hline 66 & 6 & 0 & 1.834587 & 3.397352 & 0.355777 \\
\hline 67 & 1 & 0 & 1.726649 & 4.264409 & 0.729924 \\
\hline 68 & 6 & 0 & 0.840453 & 2.530007 & -0.043764 \\
\hline
\end{tabular}




\begin{tabular}{|c|c|c|c|c|c|}
\hline 69 & 6 & 0 & -0.606836 & 2.570717 & -0.073049 \\
\hline 70 & 6 & 0 & -1.561695 & 3.550179 & 0.089004 \\
\hline 71 & 1 & 0 & -1.420052 & 4.455210 & 0.342164 \\
\hline 72 & 6 & 0 & -2.771673 & 2.939801 & -0.195613 \\
\hline 73 & 6 & 0 & -4.116980 & 3.664342 & -0.196517 \\
\hline 74 & 6 & 0 & -3.892583 & 5.076792 & -0.757656 \\
\hline 75 & 1 & 0 & -3.260206 & 5.559246 & -0.184566 \\
\hline 76 & 1 & 0 & -4.744350 & 5.557512 & -0.781458 \\
\hline 77 & 1 & 0 & -3.528311 & 5.012586 & -1.665739 \\
\hline 78 & 6 & 0 & -5.156054 & 3.001292 & -1.091700 \\
\hline 79 & 1 & 0 & -4.790704 & 2.902973 & -1.997300 \\
\hline 80 & 1 & 0 & -5.962652 & 3.556377 & -1.122683 \\
\hline 81 & 1 & 0 & -5.379441 & 2.117355 & -0.732785 \\
\hline 82 & 6 & 0 & -4.623753 & 3.779256 & 1.240920 \\
\hline 83 & 1 & 0 & -4.761864 & 2.882825 & 1.611910 \\
\hline 84 & 1 & 0 & -5.470350 & 4.271207 & 1.250780 \\
\hline 85 & 1 & 0 & -3.961145 & 4.257847 & 1.783266 \\
\hline 86 & 13 & 0 & -3.725724 & 0.058350 & -0.762215 \\
\hline 87 & 13 & 0 & 0.039984 & 0.047236 & -1.138937 \\
\hline 88 & 13 & 0 & 3.697915 & 0.024450 & -1.310287 \\
\hline 89 & 7 & 0 & -2.572732 & -1.538291 & -0.566604 \\
\hline 90 & 7 & 0 & -1.208357 & -1.370450 & -0.553621 \\
\hline 91 & 7 & 0 & 1.353043 & -1.380324 & -0.660409 \\
\hline 92 & 7 & 0 & 2.727753 & -1.493804 & -0.521639 \\
\hline 93 & 7 & 0 & 2.751139 & 1.512467 & -0.427857 \\
\hline 94 & 7 & 0 & 1.378906 & 1.398501 & -0.514438 \\
\hline 95 & 7 & 0 & -1.186470 & 1.417887 & -0.417015 \\
\hline 96 & 7 & 0 & -2.544614 & 1.620894 & -0.490049 \\
\hline 97 & 1 & 0 & -1.134647 & 0.172514 & -3.328678 \\
\hline 98 & 1 & 0 & 0.224929 & 0.997273 & -3.458510 \\
\hline 99 & 1 & 0 & 0.179674 & -0.474229 & -3.579411 \\
\hline 100 & 6 & 0 & 1.883300 & -0.077319 & 2.697209 \\
\hline 101 & 1 & 0 & 2.103240 & 0.723549 & 2.177990 \\
\hline 102 & 1 & 0 & 2.207659 & -0.872475 & 2.224663 \\
\hline 103 & 1 & 0 & 2.312782 & -0.024025 & 3.578301 \\
\hline 104 & 6 & 0 & 0.434123 & -0.167771 & 2.864375 \\
\hline 105 & 6 & 0 & -0.357340 & 0.947774 & 2.975360 \\
\hline 106 & 1 & 0 & 0.052668 & 1.802941 & 2.912341 \\
\hline
\end{tabular}




$\begin{array}{lllrrr}107 & 6 & 0 & -1.723233 & 0.884913 & 3.172131 \\ 108 & 1 & 0 & -2.237159 & 1.682561 & 3.227469 \\ 109 & 6 & 0 & -2.319938 & -0.309668 & 3.286610 \\ 110 & 1 & 0 & -3.253719 & -0.361123 & 3.454274 \\ 111 & 6 & 0 & -1.568879 & -1.470961 & 3.156247 \\ 112 & 1 & 0 & -2.005009 & -2.313291 & 3.205596 \\ 113 & 6 & 0 & -0.207577 & -1.431585 & 2.956579 \\ 114 & 1 & 0 & 0.293268 & -2.233793 & 2.880917\end{array}$

Table S6. Mulliken charge of $\mathrm{D}^{\mathrm{Bu}}{ }_{2} \mathrm{Al}_{3} \mathrm{Me}_{5}$

$\begin{array}{rlc} & & 1 \\ 1 & \mathrm{C} & -0.749018 \\ 2 & \mathrm{H} & 0.166299 \\ 3 & \mathrm{H} & 0.165686 \\ 4 & \mathrm{H} & 0.164442 \\ 5 & \mathrm{C} & -0.796331 \\ 6 & \mathrm{H} & 0.168187 \\ 7 & \mathrm{H} & 0.166824 \\ 8 & \mathrm{H} & 0.171900 \\ 9 & \mathrm{C} & -0.715520 \\ 10 & \mathrm{C} & -0.796548 \\ 11 & \mathrm{H} & 0.169130 \\ 12 & \mathrm{H} & 0.163863 \\ 13 & \mathrm{H} & 0.172824 \\ 14 & \mathrm{C} & -0.743313 \\ 15 & \mathrm{H} & 0.172707 \\ 16 & \mathrm{H} & 0.158272 \\ 17 & \mathrm{H} & 0.169831 \\ 18 & \mathrm{C} & 0.355688 \\ 19 & \mathrm{C} & -0.061970 \\ 20 & \mathrm{C} & -0.473293 \\ 21 & \mathrm{H} & 0.151538 \\ 22 & \mathrm{H} & 0.177151 \\ 23 & \mathrm{H} & 0.197726 \\ 24 & \mathrm{C} & -0.449139 \\ 25 & \mathrm{H} & 0.152447 \\ 26 & \mathrm{H} & 0.151590\end{array}$




\begin{tabular}{|c|c|c|}
\hline & $\mathrm{H}$ & \\
\hline & $\mathrm{C}$ & -0.443599 \\
\hline & $\mathrm{H}$ & 0.153704 \\
\hline & $\mathrm{H}$ & 0.162432 \\
\hline & $\mathrm{H}$ & 0.152385 \\
\hline & $\mathrm{C}$ & -0.305767 \\
\hline & $\mathrm{H}$ & 0.103583 \\
\hline & $\mathrm{C}$ & \\
\hline & $\mathrm{C}$ & 0.354745 \\
\hline & $\mathrm{C}$ & -0.305850 \\
\hline & $\mathrm{H}$ & 0.104308 \\
\hline & $\mathrm{C}$ & 0.3614 \\
\hline & $\mathrm{C}$ & -0.059769 \\
\hline & $\mathrm{C}$ & -0.446956 \\
\hline & $\mathrm{H}$ & 0.154790 \\
\hline & $\mathrm{H}$ & 0.155917 \\
\hline & $\mathrm{H}$ & 0.157218 \\
\hline & $\mathrm{C}$ & -0.450402 \\
\hline & $\mathrm{H}$ & 0.157455 \\
\hline & $\mathrm{H}$ & 0.151047 \\
\hline & $\mathrm{H}$ & 0.186197 \\
\hline & $\mathrm{C}$ & -0.454828 \\
\hline & $\mathrm{H}$ & 0.179839 \\
\hline & $\mathrm{H}$ & 0.151877 \\
\hline & $\mathrm{H}$ & 0.156140 \\
\hline & $\mathrm{C}$ & 0.361113 \\
\hline & $\mathrm{C}$ & -0.057862 \\
\hline & $\mathrm{C}$ & -0.452541 \\
\hline & $\mathrm{H}$ & 0.154851 \\
\hline & $\mathrm{H}$ & 0.152299 \\
\hline & $\mathrm{H}$ & 0.178836 \\
\hline & $\mathrm{C}$ & -0.450747 \\
\hline & $\mathrm{H}$ & 0.184971 \\
\hline & $\mathrm{H}$ & 0.150666 \\
\hline & $\mathrm{H}$ & 0.158527 \\
\hline & $\mathrm{C}$ & -0.448516 \\
\hline & $\mathrm{H}$ & 0.157995 \\
\hline & $\mathrm{H}$ & 0.15578 \\
\hline
\end{tabular}




\begin{tabular}{|c|c|c|}
\hline 65 & $\mathrm{H}$ & 0.155189 \\
\hline 66 & $\mathrm{C}$ & -0.308836 \\
\hline 67 & $\mathrm{H}$ & 0.104120 \\
\hline 68 & $\mathrm{C}$ & 0.352609 \\
\hline 69 & $\mathrm{C}$ & 0.351138 \\
\hline 70 & $\mathrm{C}$ & -0.299645 \\
\hline 71 & $\mathrm{H}$ & 0.103986 \\
\hline 72 & $\mathrm{C}$ & 0.350445 \\
\hline 73 & $\mathrm{C}$ & -0.059764 \\
\hline 74 & $\mathrm{C}$ & -0.441688 \\
\hline 75 & $\mathrm{H}$ & 0.152673 \\
\hline 76 & $\mathrm{H}$ & 0.151813 \\
\hline 77 & $\mathrm{H}$ & 0.163785 \\
\hline 78 & $\mathrm{C}$ & -0.475688 \\
\hline 79 & $\mathrm{H}$ & 0.179568 \\
\hline 80 & $\mathrm{H}$ & 0.151644 \\
\hline 81 & $\mathrm{H}$ & 0.197704 \\
\hline 82 & $\mathrm{C}$ & -0.446932 \\
\hline 83 & $\mathrm{H}$ & 0.179304 \\
\hline 84 & $\mathrm{H}$ & 0.152024 \\
\hline 85 & $\mathrm{H}$ & 0.151072 \\
\hline 86 & $\mathrm{Al}$ & 0.826886 (outer) \\
\hline 87 & $\mathrm{Al}$ & 0.929040 (central) \\
\hline 88 & $\mathrm{Al}$ & 0.845585 (outer) \\
\hline 89 & $\mathrm{~N}$ & -0.451236 \\
\hline 90 & $\mathrm{~N}$ & -0.416704 \\
\hline 91 & $\mathrm{~N}$ & -0.433960 \\
\hline 92 & $\mathrm{~N}$ & -0.462818 \\
\hline 93 & $\mathrm{~N}$ & -0.462163 \\
\hline 94 & $\mathrm{~N}$ & -0.432517 \\
\hline 95 & $\mathrm{~N}$ & -0.414913 \\
\hline 96 & $\mathrm{~N}$ & -0.452282 \\
\hline 97 & $\mathrm{H}$ & 0.162201 \\
\hline 98 & $\mathrm{H}$ & 0.153871 \\
\hline 99 & $\mathrm{H}$ & 0.161922 \\
\hline 100 & $\mathrm{C}$ & -0.521499 \\
\hline 101 & $\mathrm{H}$ & 0.181318 \\
\hline 102 & $\mathrm{H}$ & 0.177381 \\
\hline
\end{tabular}




$\begin{array}{ccc}103 & \mathrm{H} & 0.168041 \\ 104 & \mathrm{C} & 0.116086 \\ 105 & \mathrm{C} & -0.158318 \\ 106 & \mathrm{H} & 0.088525 \\ 107 & \mathrm{C} & -0.087652 \\ 108 & \mathrm{H} & 0.103521 \\ 109 & \mathrm{C} & -0.101491 \\ 110 & \mathrm{H} & 0.104449 \\ 111 & \mathrm{C} & -0.102943 \\ 112 & \mathrm{H} & 0.101163 \\ 113 & \mathrm{C} & -0.151800 \\ 114 & \mathrm{H} & 0.091593\end{array}$


CL

Table S7. Atomic coordinates of CL

\begin{tabular}{|c|c|c|c|c|}
\hline \multirow{3}{*}{$\begin{array}{l}\text { Center } \\
\text { Number } \\
\text { Z }\end{array}$} & \multirow{3}{*}{$\begin{array}{l}\text { Atomic } \\
\text { Number }\end{array}$} & \multicolumn{3}{|c|}{ Coordinates (Angstroms) } \\
\hline & & $\mathrm{X}$ & \multicolumn{2}{|l|}{$\mathrm{Y}$} \\
\hline & & & & \\
\hline 1 & 8 & -1.894317 & -0.481581 & 0.848453 \\
\hline 2 & 6 & -1.224007 & -0.080983 & -0.070478 \\
\hline 3 & 6 & -0.917682 & 1.356834 & -0.407274 \\
\hline 4 & 6 & 0.474064 & 1.689102 & 0.200162 \\
\hline 5 & 1 & -0.886063 & 1.474114 & -1.495421 \\
\hline 6 & 1 & -1.680806 & 2.019923 & 0.008944 \\
\hline 7 & 6 & 0.529386 & -1.655408 & -0.209008 \\
\hline 8 & 6 & 1.616018 & 0.687996 & -0.093320 \\
\hline 9 & 1 & 0.360769 & 1.778308 & 1.288797 \\
\hline 10 & 1 & 0.770431 & 2.680012 & -0.164462 \\
\hline 11 & 6 & 1.454950 & -0.702403 & 0.568196 \\
\hline 12 & 1 & 1.067732 & -2.167507 & -1.010541 \\
\hline 13 & 1 & 0.076223 & -2.405628 & 0.448349 \\
\hline 14 & 1 & 1.746470 & 0.573475 & -1.177329 \\
\hline 15 & 1 & 2.541875 & 1.138843 & 0.284684 \\
\hline 16 & 1 & 2.435885 & -1.183552 & 0.668146 \\
\hline 17 & 1 & 1.073769 & -0.568224 & 1.589034 \\
\hline 18 & 8 & -0.493517 & -0.907242 & -0.894687 \\
\hline
\end{tabular}


Table S8. Thermochemistry data of CL

Temperature 298.150 Kelvin. Pressure 1.00000 Atm.

Molecular mass: $\quad 114.06808 \mathrm{amu}$.

\begin{tabular}{|c|c|c|}
\hline & (Hartree/Particle) & $(\mathrm{kcal} / \mathrm{mol})$ \\
\hline Zero-point correction $=$ & 0.157114 & \\
\hline Thermal correction to Energy= & 0.164421 & \\
\hline Thermal correction to Enthalpy= & 0.165365 & \\
\hline Thermal correction to Gibbs Free Energy= & 0.125535 & \\
\hline Electronic Energy= & -385.106406 & -241657.918 \\
\hline Sum of electronic and zero-point Energies= & -384.949292 & -241559.327 \\
\hline Sum of electronic and thermal Energies= & -384.941985 & -241554.742 \\
\hline Sum of electronic and thermal Enthalpies= & -384.941041 & -241554.150 \\
\hline Sum of electronic and thermal Free Energies $=$ & $=-384.980871$ & -241579.144 \\
\hline E (Thermal) & $\mathrm{CV}$ & $\mathrm{S}$ \\
\hline $\mathrm{KCal} / \mathrm{Mol}$ & Cal/Mol-Kelvin & $\mathrm{Cal} / \mathrm{Mol}-\mathrm{Kelvin}$ \\
\hline 103.176 & 27.690 & 83.829 \\
\hline
\end{tabular}




\section{Cat}

Table S9. Atomic coordinates of cat

$\begin{array}{lcccc}\text { Center } & \text { Atomic } & \text { Atomic } & \text { Coordinates (Angstroms) } \\ \text { Number } & \text { Number } & \text { Type } & X & \text { Y } \\ Z & & & \end{array}$

\begin{tabular}{|c|c|c|c|c|c|}
\hline 1 & 13 & 0 & 0.025335 & -0.005815 & 0.511695 \\
\hline 2 & 7 & 0 & -1.238482 & -1.427573 & -0.114867 \\
\hline 3 & 6 & 0 & -0.661288 & -2.630205 & -0.280810 \\
\hline 4 & 6 & 0 & -2.852565 & -2.946578 & -0.127303 \\
\hline 5 & 6 & 0 & -1.637941 & -3.618734 & -0.300441 \\
\hline 6 & 1 & 0 & -1.488463 & -4.681604 & -0.399107 \\
\hline 7 & 7 & 0 & 1.333664 & -1.401617 & -0.048374 \\
\hline 8 & 6 & 0 & 0.789609 & -2.599679 & -0.326486 \\
\hline 9 & 6 & 0 & 2.993585 & -2.805646 & -0.463142 \\
\hline 10 & 6 & 0 & 1.796322 & -3.517009 & -0.596718 \\
\hline 11 & 1 & 0 & 1.679733 & -4.555319 & -0.861111 \\
\hline 12 & 7 & 0 & -1.270240 & 1.404186 & -0.071904 \\
\hline 13 & 6 & 0 & -0.719405 & 2.623457 & -0.201936 \\
\hline 14 & 6 & 0 & -1.716431 & 3.592012 & -0.170599 \\
\hline 15 & 6 & 0 & -2.914085 & 2.888690 & -0.003090 \\
\hline 16 & 1 & 0 & -1.590236 & 4.660982 & -0.228344 \\
\hline 17 & 7 & 0 & 1.303422 & 1.431073 & -0.020987 \\
\hline 18 & 6 & 0 & 0.731126 & 2.623020 & -0.267292 \\
\hline 19 & 6 & 0 & 2.927858 & 2.876403 & -0.438365 \\
\hline 20 & 6 & 0 & 1.713901 & 3.564978 & -0.537871 \\
\hline 21 & 1 & 0 & 1.571313 & 4.604756 & -0.782677 \\
\hline 22 & 7 & 0 & -2.629860 & 1.548427 & 0.048267 \\
\hline 23 & 7 & 0 & -2.596181 & -1.604246 & -0.020401 \\
\hline 24 & 7 & 0 & 2.697715 & -1.510145 & -0.132683 \\
\hline 25 & 7 & 0 & 2.663762 & 1.569388 & -0.125013 \\
\hline 26 & 13 & 0 & -3.719451 & -0.033171 & -0.351581 \\
\hline 27 & 13 & 0 & 3.739877 & 0.040187 & 0.488852 \\
\hline 28 & 6 & 0 & -4.299735 & 3.501680 & 0.173269 \\
\hline 29 & 6 & 0 & -4.226615 & -3.599085 & -0.006406 \\
\hline 30 & 6 & 0 & 4.309956 & 3.486276 & -0.650292 \\
\hline
\end{tabular}




\begin{tabular}{|c|c|c|c|c|c|}
\hline 31 & 6 & 0 & 4.394257 & -3.376656 & -0.654292 \\
\hline 32 & 6 & 0 & -5.238993 & 3.117051 & -0.992716 \\
\hline 33 & 1 & 0 & -5.376438 & 2.039328 & -1.091839 \\
\hline 34 & 1 & 0 & -6.224061 & 3.571895 & -0.835569 \\
\hline 35 & 1 & 0 & -4.843799 & 3.487805 & -1.945564 \\
\hline 36 & 6 & 0 & -4.889487 & 3.052325 & 1.530862 \\
\hline 37 & 1 & 0 & -5.881701 & 3.498645 & 1.669421 \\
\hline 38 & 1 & 0 & -4.981690 & 1.965800 & 1.587622 \\
\hline 39 & 1 & 0 & -4.251337 & 3.388142 & 2.356669 \\
\hline 40 & 6 & 0 & -4.180312 & 5.040760 & 0.187522 \\
\hline 41 & 1 & 0 & -3.537618 & 5.390704 & 1.002855 \\
\hline 42 & 1 & 0 & -3.784649 & 5.428905 & -0.758015 \\
\hline 43 & 1 & 0 & -5.173365 & 5.478462 & 0.335503 \\
\hline 44 & 6 & 0 & -5.173157 & -3.140902 & -1.138585 \\
\hline 45 & 1 & 0 & -5.370552 & -2.068913 & -1.117441 \\
\hline 46 & 1 & 0 & -4.752320 & -3.379507 & -2.121577 \\
\hline 47 & 1 & 0 & -6.133681 & -3.661183 & -1.043754 \\
\hline 48 & 6 & 0 & -4.830689 & -3.273619 & 1.380208 \\
\hline 49 & 1 & 0 & -4.186391 & -3.656464 & 2.180417 \\
\hline 50 & 1 & 0 & -4.952046 & -2.197201 & 1.518248 \\
\hline 51 & 1 & 0 & -5.811370 & -3.754922 & 1.478477 \\
\hline 52 & 6 & 0 & -4.073490 & -5.131308 & -0.117480 \\
\hline 53 & 1 & 0 & -3.659024 & -5.430977 & -1.086861 \\
\hline 54 & 1 & 0 & -3.432197 & -5.534284 & 0.674176 \\
\hline 55 & 1 & 0 & -5.057818 & -5.601082 & -0.018138 \\
\hline 56 & 6 & 0 & 5.000777 & 2.837521 & -1.872566 \\
\hline 57 & 1 & 0 & 4.398834 & 2.986589 & -2.776957 \\
\hline 58 & 1 & 0 & 5.976565 & 3.310785 & -2.038036 \\
\hline 59 & 1 & 0 & 5.152289 & 1.767315 & -1.721109 \\
\hline 60 & 6 & 0 & 5.168768 & 3.331894 & 0.625247 \\
\hline 61 & 1 & 0 & 5.324946 & 2.287342 & 0.895159 \\
\hline 62 & 1 & 0 & 6.152883 & 3.788658 & 0.466213 \\
\hline 63 & 1 & 0 & 4.694935 & 3.833470 & 1.476502 \\
\hline 64 & 6 & 0 & 4.163990 & 4.997402 & -0.933208 \\
\hline 65 & 1 & 0 & 3.587351 & 5.187237 & -1.845422 \\
\hline 66 & 1 & 0 & 3.683539 & 5.525315 & -0.101652 \\
\hline 67 & 1 & 0 & 5.158600 & 5.433873 & -1.074162 \\
\hline 68 & 6 & 0 & 5.088830 & -2.696566 & -1.857143 \\
\hline
\end{tabular}




\begin{tabular}{|c|c|c|c|c|c|}
\hline 69 & 1 & 0 & 6.084901 & -3.132367 & -2.002874 \\
\hline 70 & 1 & 0 & 4.512953 & -2.862166 & -2.775407 \\
\hline 71 & 1 & 0 & 5.196077 & -1.621966 & -1.698233 \\
\hline 72 & 6 & 0 & 5.223281 & -3.208231 & 0.639463 \\
\hline 73 & 1 & 0 & 6.227237 & -3.624333 & 0.494268 \\
\hline 74 & 1 & 0 & 5.335131 & -2.162859 & 0.930364 \\
\hline 75 & 1 & 0 & 4.753141 & -3.742410 & 1.473121 \\
\hline 76 & 6 & 0 & 4.295412 & -4.888430 & -0.951464 \\
\hline 77 & 1 & 0 & 3.811908 & -5.435359 & -0.134056 \\
\hline 78 & 1 & 0 & 3.742432 & -5.085720 & -1.876578 \\
\hline 79 & 1 & 0 & 5.303759 & -5.297713 & -1.075554 \\
\hline 80 & 8 & 0 & -5.087551 & -0.103709 & 0.695944 \\
\hline 81 & 8 & 0 & -4.129716 & 0.019122 & -2.028049 \\
\hline 82 & 8 & 0 & 0.012164 & -0.033230 & 2.242858 \\
\hline 83 & 8 & 0 & 3.904321 & 0.038386 & 2.194510 \\
\hline 84 & 8 & 0 & 5.250772 & 0.087807 & -0.346231 \\
\hline 85 & 6 & 0 & 6.581858 & 0.068708 & 0.108892 \\
\hline 86 & 1 & 0 & 7.108021 & -0.811157 & -0.288523 \\
\hline 87 & 1 & 0 & 7.116100 & 0.962378 & -0.243972 \\
\hline 88 & 1 & 0 & 6.649832 & 0.041316 & 1.205144 \\
\hline 89 & 6 & 0 & 3.245657 & -0.521809 & 3.305909 \\
\hline 90 & 1 & 0 & 3.643078 & -0.071750 & 4.225962 \\
\hline 91 & 1 & 0 & 2.163380 & -0.349294 & 3.270109 \\
\hline 92 & 1 & 0 & 3.419485 & -1.607963 & 3.366396 \\
\hline 93 & 6 & 0 & -1.039838 & 0.000796 & 3.172226 \\
\hline 94 & 1 & 0 & -1.707460 & -0.870449 & 3.078503 \\
\hline 95 & 1 & 0 & -0.627331 & -0.007335 & 4.190457 \\
\hline 96 & 1 & 0 & -1.656858 & 0.907696 & 3.072475 \\
\hline 97 & 6 & 0 & -6.475190 & -0.076224 & 0.467479 \\
\hline 98 & 1 & 0 & -6.929166 & 0.783572 & 0.980395 \\
\hline 99 & 1 & 0 & -6.728514 & -0.005445 & -0.599816 \\
\hline 100 & 1 & 0 & -6.943581 & -0.987225 & 0.866325 \\
\hline 101 & 6 & 0 & -3.438583 & 0.436776 & -3.175038 \\
\hline 102 & 1 & 0 & -2.663451 & -0.285844 & -3.475398 \\
\hline 103 & 1 & 0 & -4.141320 & 0.534646 & -4.013884 \\
\hline 104 & 1 & 0 & -2.947709 & 1.413710 & -3.037230 \\
\hline
\end{tabular}


Table S10. Thermochemistry data of cat

Temperature 298.150 Kelvin. Pressure 1.00000 Atm.

Molecular mass: $\quad 724.37417 \mathrm{amu}$.

\begin{tabular}{|c|c|c|}
\hline & (Hartree/Particle) & $(\mathrm{kcal} / \mathrm{mol})$ \\
\hline Zero-point correction $=$ & 0.874928 & \\
\hline Thermal correction to Energy $=$ & 0.932544 & \\
\hline Thermal correction to Enthalpy= & 0.933488 & \\
\hline Thermal correction to Gibbs Free Energy= & 0.782906 & \\
\hline Electronic Energy= & -2832.590293 & -1777477.245 \\
\hline Sum of electronic and zero-point Energies= & -2831.715365 & -1776928.219 \\
\hline Sum of electronic and thermal Energies= & -2831.657749 & -1776892.065 \\
\hline Sum of electronic and thermal Enthalpies $=$ & -2831.656805 & -1776891.472 \\
\hline \multicolumn{2}{|l|}{ Sum of electronic and thermal Free Energies= } & -2831.807387 \\
\hline & -1776985.964 & \\
\hline E (Thermal) & $\mathrm{CV}$ & $\mathrm{S}$ \\
\hline $\mathrm{KCal} / \mathrm{Mol}$ & Cal/Mol-Kelvin & Cal/Mol-Kelvin \\
\hline 585.180 & 212.404 & 316.928 \\
\hline
\end{tabular}


Intermediate I

Table S11. Atomic coordinates of I

$\begin{array}{lcccc}\text { Center } & \text { Atomic } & \text { Atomic } & \text { Coordinates (Angstroms) } \\ \text { Number } & \text { Number } & \text { Type } & X & \text { Y } \\ Z & & & \end{array}$

\begin{tabular}{|c|c|c|c|c|c|}
\hline 1 & 13 & 0 & -0.065807 & -0.010478 & 0.379748 \\
\hline 2 & 7 & 0 & 1.239358 & 1.450078 & 0.026918 \\
\hline 3 & 6 & 0 & 0.670956 & 2.657669 & -0.113847 \\
\hline 4 & 6 & 0 & 2.842274 & 2.963676 & 0.231798 \\
\hline 5 & 6 & 0 & 1.643208 & 3.645766 & 0.005452 \\
\hline 6 & 1 & 0 & 1.498608 & 4.713016 & -0.039672 \\
\hline 7 & 7 & 0 & -1.333645 & 1.418635 & -0.171530 \\
\hline 8 & 6 & 0 & -0.770825 & 2.631511 & -0.292621 \\
\hline 9 & 6 & 0 & -2.957994 & 2.858618 & -0.600296 \\
\hline 10 & 6 & 0 & -1.756838 & 3.573448 & -0.567617 \\
\hline 11 & 1 & 0 & -1.623132 & 4.632547 & -0.716857 \\
\hline 12 & 7 & 0 & 1.277779 & -1.411996 & -0.065724 \\
\hline 13 & 6 & 0 & 0.741691 & -2.627440 & -0.261483 \\
\hline 14 & 6 & 0 & 1.742486 & -3.592211 & -0.198943 \\
\hline 15 & 6 & 0 & 2.923488 & -2.888276 & 0.054748 \\
\hline 16 & 1 & 0 & 1.627837 & -4.659715 & -0.295707 \\
\hline 17 & 7 & 0 & -1.296632 & -1.444175 & -0.240389 \\
\hline 18 & 6 & 0 & -0.701960 & -2.633117 & -0.428072 \\
\hline 19 & 6 & 0 & -2.882858 & -2.900804 & -0.748175 \\
\hline 20 & 6 & 0 & -1.663180 & -3.584570 & -0.754052 \\
\hline 21 & 1 & 0 & -1.501776 & -4.629467 & -0.964035 \\
\hline 22 & 7 & 0 & 2.626061 & -1.550262 & 0.128327 \\
\hline 23 & 7 & 0 & 2.582083 & 1.615994 & 0.236145 \\
\hline 24 & 7 & 0 & -2.683427 & 1.535050 & -0.362918 \\
\hline 25 & 7 & 0 & -2.642595 & -1.585414 & -0.438124 \\
\hline 26 & 13 & 0 & 3.729478 & 0.060263 & -0.099806 \\
\hline 27 & 13 & 0 & -3.785688 & -0.049037 & 0.014007 \\
\hline 28 & 6 & 0 & 4.301750 & -3.499882 & 0.285302 \\
\hline 29 & 6 & 0 & 4.200887 & 3.604250 & 0.498878 \\
\hline 30 & 6 & 0 & -4.247450 & -3.514651 & -1.045585 \\
\hline
\end{tabular}




\begin{tabular}{|c|c|c|c|c|c|}
\hline 31 & 6 & 0 & -4.338814 & 3.454706 & -0.857607 \\
\hline 32 & 6 & 0 & 5.303876 & -3.054978 & -0.804458 \\
\hline 33 & 1 & 0 & 5.417232 & -1.971548 & -0.865914 \\
\hline 34 & 1 & 0 & 6.289100 & -3.489530 & -0.597784 \\
\hline 35 & 1 & 0 & 4.980026 & -3.406853 & -1.790957 \\
\hline 36 & 6 & 0 & 4.806175 & -3.114854 & 1.695971 \\
\hline 37 & 1 & 0 & 5.792377 & -3.561950 & 1.871089 \\
\hline 38 & 1 & 0 & 4.884610 & -2.032116 & 1.812299 \\
\hline 39 & 1 & 0 & 4.122787 & -3.495909 & 2.464018 \\
\hline 40 & 6 & 0 & 4.196559 & -5.038833 & 0.220154 \\
\hline 41 & 1 & 0 & 3.513959 & -5.431792 & 0.981756 \\
\hline 42 & 1 & 0 & 3.856485 & -5.384253 & -0.762873 \\
\hline 43 & 1 & 0 & 5.184300 & -5.476081 & 0.401875 \\
\hline 44 & 6 & 0 & 5.223282 & 3.236599 & -0.600155 \\
\hline 45 & 1 & 0 & 5.396719 & 2.163062 & -0.679108 \\
\hline 46 & 1 & 0 & 4.880114 & 3.584922 & -1.580917 \\
\hline 47 & 1 & 0 & 6.183266 & 3.720686 & -0.383922 \\
\hline 48 & 6 & 0 & 4.710478 & 3.174824 & 1.894887 \\
\hline 49 & 1 & 0 & 4.008401 & 3.493425 & 2.674486 \\
\hline 50 & 1 & 0 & 4.831255 & 2.091680 & 1.960239 \\
\hline 51 & 1 & 0 & 5.677431 & 3.651427 & 2.097724 \\
\hline 52 & 6 & 0 & 4.049892 & 5.140864 & 0.497867 \\
\hline 53 & 1 & 0 & 3.706265 & 5.517329 & -0.472433 \\
\hline 54 & 1 & 0 & 3.351102 & 5.481273 & 1.270037 \\
\hline 55 & 1 & 0 & 5.023143 & 5.598587 & 0.705467 \\
\hline 56 & 6 & 0 & -4.815257 & -2.936434 & -2.363417 \\
\hline 57 & 1 & 0 & -4.147565 & -3.170884 & -3.201959 \\
\hline 58 & 1 & 0 & -5.790360 & -3.390949 & -2.577750 \\
\hline 59 & 1 & 0 & -4.936672 & -1.853289 & -2.301840 \\
\hline 60 & 6 & 0 & -5.220805 & -3.279617 & 0.131324 \\
\hline 61 & 1 & 0 & -5.385005 & -2.221931 & 0.339086 \\
\hline 62 & 1 & 0 & -6.192714 & -3.732842 & -0.097355 \\
\hline 63 & 1 & 0 & -4.838109 & -3.742188 & 1.047978 \\
\hline 64 & 6 & 0 & -4.094827 & -5.040892 & -1.223768 \\
\hline 65 & 1 & 0 & -3.446831 & -5.289537 & -2.071687 \\
\hline 66 & 1 & 0 & -3.688878 & -5.518471 & -0.325011 \\
\hline 67 & 1 & 0 & -5.078663 & -5.480445 & -1.420583 \\
\hline 68 & 6 & 0 & -4.904172 & 2.932462 & -2.198935 \\
\hline
\end{tabular}




\begin{tabular}{|c|c|c|c|c|c|}
\hline 69 & 1 & 0 & -5.891512 & 3.373896 & -2.382555 \\
\hline 70 & 1 & 0 & -4.249031 & 3.224232 & -3.029143 \\
\hline 71 & 1 & 0 & -4.999644 & 1.845099 & -2.191647 \\
\hline 72 & 6 & 0 & -5.298197 & 3.136638 & 0.311453 \\
\hline 73 & 1 & 0 & -6.279704 & 3.584588 & 0.115556 \\
\hline 74 & 1 & 0 & -5.442765 & 2.066156 & 0.460691 \\
\hline 75 & 1 & 0 & -4.915991 & 3.554157 & 1.249736 \\
\hline 76 & 6 & 0 & -4.222686 & 4.991243 & -0.957086 \\
\hline 77 & 1 & 0 & -3.825311 & 5.431262 & -0.035603 \\
\hline 78 & 1 & 0 & -3.583195 & 5.298439 & -1.792206 \\
\hline 79 & 1 & 0 & -5.217041 & 5.417246 & -1.129225 \\
\hline 80 & 8 & 0 & 5.014574 & 0.064520 & 1.054519 \\
\hline 81 & 8 & 0 & 4.306775 & 0.110326 & -1.725717 \\
\hline 82 & 8 & 0 & -0.243306 & -0.070357 & 2.112227 \\
\hline 83 & 8 & 0 & -4.298561 & -0.096379 & 1.648034 \\
\hline 84 & 8 & 0 & -5.120572 & -0.041624 & -1.087095 \\
\hline 85 & 6 & 0 & -6.509549 & -0.064352 & -0.860037 \\
\hline 86 & 1 & 0 & -6.981299 & 0.830315 & -1.291691 \\
\hline 87 & 1 & 0 & -6.960296 & -0.942816 & -1.344169 \\
\hline 88 & 1 & 0 & -6.760937 & -0.098893 & 0.208747 \\
\hline 89 & 6 & 0 & -3.715696 & -0.092229 & 2.931055 \\
\hline 90 & 1 & 0 & -4.050378 & -0.974121 & 3.496686 \\
\hline 91 & 1 & 0 & -2.618641 & -0.099445 & 2.900061 \\
\hline 92 & 1 & 0 & -4.038595 & 0.799502 & 3.488529 \\
\hline 93 & 6 & 0 & 0.734617 & -0.055797 & 3.118352 \\
\hline 94 & 1 & 0 & 1.332505 & 0.869903 & 3.108449 \\
\hline 95 & 1 & 0 & 0.250592 & -0.124455 & 4.102700 \\
\hline 96 & 1 & 0 & 1.434024 & -0.903638 & 3.035580 \\
\hline 97 & 6 & 0 & 6.412757 & 0.064720 & 0.897397 \\
\hline 98 & 1 & 0 & 6.849768 & -0.829050 & 1.365489 \\
\hline 99 & 1 & 0 & 6.720097 & 0.078931 & -0.157563 \\
\hline 100 & 1 & 0 & 6.850058 & 0.945344 & 1.389411 \\
\hline 101 & 6 & 0 & 3.734325 & -0.167979 & -2.977010 \\
\hline 102 & 1 & 0 & 2.915432 & 0.522838 & -3.223294 \\
\hline 103 & 1 & 0 & 4.501320 & -0.074903 & -3.758459 \\
\hline 104 & 1 & 0 & 3.331770 & -1.192768 & -3.027774 \\
\hline 105 & 6 & 0 & -0.627504 & 1.999117 & -4.738761 \\
\hline 106 & 6 & 0 & -1.986539 & 1.766825 & -5.398303 \\
\hline
\end{tabular}




$\begin{array}{lllrrr}107 & 6 & 0 & -2.766556 & 0.542977 & -4.868573 \\ 108 & 6 & 0 & -0.996874 & -1.264559 & -4.199412 \\ 109 & 6 & 0 & -2.113453 & -0.825859 & -5.179004 \\ 110 & 1 & 0 & -0.724225 & 2.339310 & -3.701169 \\ 111 & 1 & 0 & -1.837558 & 1.645575 & -6.479515 \\ 112 & 1 & 0 & -2.943328 & 0.640992 & -3.787822 \\ 113 & 1 & 0 & -1.404001 & -1.757010 & -3.312280 \\ 114 & 1 & 0 & -0.033074 & 2.732064 & -5.289630 \\ 115 & 1 & 0 & -2.590801 & 2.674603 & -5.263020 \\ 116 & 1 & 0 & -3.759490 & 0.560762 & -5.333334 \\ 117 & 1 & 0 & -0.328635 & -1.982452 & -4.693429 \\ 118 & 1 & 0 & -2.887640 & -1.601740 & -5.161270 \\ 119 & 1 & 0 & -1.716367 & -0.803544 & -6.200470 \\ 120 & 6 & 0 & -0.179714 & -0.082508 & -3.734526 \\ 121 & 8 & 0 & 0.141802 & 0.148551 & -2.591588 \\ 122 & 8 & 0 & 0.137211 & 0.769124 & -4.761512\end{array}$


Table S12. Thermochemistry data of I

- Thermochemistry -

Temperature 298.150 Kelvin. Pressure 1.00000 Atm.

Molecular mass: $\quad 838.44225 \mathrm{amu}$.

\begin{tabular}{|c|c|c|}
\hline & (Hartree/Particle) & $(\mathrm{kcal} / \mathrm{mol})$ \\
\hline Zero-point correction= & 1.032518 & \\
\hline Thermal correction to Energy= & 1.099632 & \\
\hline Thermal correction to Enthalpy= & 1.100576 & \\
\hline Thermal correction to Gibbs Free Energy= & 0.927348 & \\
\hline Electronic Energy= & -3217.703163 & -2019139.219 \\
\hline Sum of electronic and zero-point Energies $=$ & -3216.670645 & -2018491.304 \\
\hline Sum of electronic and thermal Energies $=$ & -3216.603531 & -2018449.190 \\
\hline Sum of electronic and thermal Enthalpies $=$ & -3216.602587 & -2018448.597 \\
\hline \multicolumn{2}{|c|}{ Sum of electronic and thermal Free Energies $=$} & -3216.775815 \\
\hline & -2018557.299 & \\
\hline E (Thermal) & $\mathrm{CV}$ & $\mathrm{S}$ \\
\hline $\mathrm{KCal} / \mathrm{Mol}$ & Cal/Mol-Kelvin & Cal/Mol-Kelvin \\
\hline 690.030 & 246.036 & 364.589 \\
\hline
\end{tabular}


Transition state I

Table S13. Atomic coordinates of TS1

$\begin{array}{lcccc}\text { Center } & \text { Atomic } & \text { Atomic } & \text { Coordinates (Angstroms) } \\ \text { Number } & \text { Number } & \text { Type } & X & \text { Y } \\ Z & & & \end{array}$

\begin{tabular}{|c|c|c|c|c|c|}
\hline 1 & 13 & 0 & -0.528022 & 0.089373 & 0.236627 \\
\hline 2 & 7 & 0 & 0.681168 & 1.609643 & -0.192427 \\
\hline 3 & 6 & 0 & 0.062549 & 2.803790 & -0.234165 \\
\hline 4 & 6 & 0 & 2.247467 & 3.160389 & -0.195413 \\
\hline 5 & 6 & 0 & 1.010495 & 3.817260 & -0.234428 \\
\hline 6 & 1 & 0 & 0.831315 & 4.880131 & -0.252382 \\
\hline 7 & 7 & 0 & -1.896549 & 1.497653 & -0.158308 \\
\hline 8 & 6 & 0 & -1.386094 & 2.737048 & -0.257472 \\
\hline 9 & 6 & 0 & -3.596373 & 2.908193 & -0.327192 \\
\hline 10 & 6 & 0 & -2.418709 & 3.659723 & -0.370479 \\
\hline 11 & 1 & 0 & -2.328877 & 4.728687 & -0.474645 \\
\hline 12 & 7 & 0 & 0.770763 & -1.266889 & -0.480490 \\
\hline 13 & 6 & 0 & 0.231597 & -2.495237 & -0.546678 \\
\hline 14 & 6 & 0 & 1.232682 & -3.452608 & -0.421657 \\
\hline 15 & 6 & 0 & 2.417728 & -2.726613 & -0.253512 \\
\hline 16 & 1 & 0 & 1.111563 & -4.523551 & -0.397568 \\
\hline 17 & 7 & 0 & -1.807212 & -1.338850 & -0.345321 \\
\hline 18 & 6 & 0 & -1.217639 & -2.514416 & -0.620762 \\
\hline 19 & 6 & 0 & -3.407364 & -2.789976 & -0.834253 \\
\hline 20 & 6 & 0 & -2.184635 & -3.460361 & -0.935721 \\
\hline 21 & 1 & 0 & -2.024184 & -4.488751 & -1.215482 \\
\hline 22 & 7 & 0 & 2.128067 & -1.387125 & -0.322713 \\
\hline 23 & 7 & 0 & 2.033423 & 1.810820 & -0.177933 \\
\hline 24 & 7 & 0 & -3.265821 & 1.584749 & -0.196343 \\
\hline 25 & 7 & 0 & -3.164164 & -1.490510 & -0.472444 \\
\hline 26 & 13 & 0 & 3.123970 & 0.257443 & -0.895068 \\
\hline 27 & 13 & 0 & -4.280450 & -0.037392 & 0.237236 \\
\hline 28 & 6 & 0 & 3.775664 & -3.324339 & 0.106394 \\
\hline 29 & 6 & 0 & 3.609215 & 3.844082 & -0.126486 \\
\hline 30 & 6 & 0 & -4.778334 & -3.403717 & -1.101267 \\
\hline
\end{tabular}




\begin{tabular}{|c|c|c|c|c|c|}
\hline 31 & 6 & 0 & -5.011658 & 3.468334 & -0.425145 \\
\hline 32 & 6 & 0 & 4.835301 & -3.071147 & -0.985149 \\
\hline 33 & 1 & 0 & 4.985960 & -2.008899 & -1.168291 \\
\hline 34 & 1 & 0 & 5.791582 & -3.513491 & -0.681049 \\
\hline 35 & 1 & 0 & 4.535425 & -3.533788 & -1.933325 \\
\hline 36 & 6 & 0 & 4.229511 & -2.726210 & 1.459065 \\
\hline 37 & 1 & 0 & 5.216744 & -3.119021 & 1.731545 \\
\hline 38 & 1 & 0 & 4.280095 & -1.637294 & 1.411770 \\
\hline 39 & 1 & 0 & 3.525071 & -2.999410 & 2.253320 \\
\hline 40 & 6 & 0 & 3.639308 & -4.852578 & 0.276575 \\
\hline 41 & 1 & 0 & 2.918810 & -5.112038 & 1.059586 \\
\hline 42 & 1 & 0 & 3.329934 & -5.341062 & -0.654792 \\
\hline 43 & 1 & 0 & 4.609758 & -5.272080 & 0.563948 \\
\hline 44 & 6 & 0 & 4.532693 & 3.368859 & -1.267408 \\
\hline 45 & 1 & 0 & 4.780978 & 2.313064 & -1.152361 \\
\hline 46 & 1 & 0 & 4.064286 & 3.514799 & -2.247143 \\
\hline 47 & 1 & 0 & 5.472631 & 3.933828 & -1.248489 \\
\hline 48 & 6 & 0 & 4.254869 & 3.550951 & 1.248115 \\
\hline 49 & 1 & 0 & 3.639151 & 3.961433 & 2.057036 \\
\hline 50 & 1 & 0 & 4.357426 & 2.475108 & 1.400750 \\
\hline 51 & 1 & 0 & 5.245841 & 4.018708 & 1.307079 \\
\hline 52 & 6 & 0 & 3.430377 & 5.370009 & -0.268962 \\
\hline 53 & 1 & 0 & 2.973204 & 5.638741 & -1.228288 \\
\hline 54 & 1 & 0 & 2.813127 & 5.783653 & 0.535959 \\
\hline 55 & 1 & 0 & 4.410230 & 5.857511 & -0.216431 \\
\hline 56 & 6 & 0 & -5.456568 & -2.694600 & -2.296705 \\
\hline 57 & 1 & 0 & -4.838299 & -2.787002 & -3.197790 \\
\hline 58 & 1 & 0 & -6.424821 & -3.166630 & -2.505069 \\
\hline 59 & 1 & 0 & -5.619689 & -1.635568 & -2.088391 \\
\hline 60 & 6 & 0 & -5.661324 & -3.332558 & 0.165050 \\
\hline 61 & 1 & 0 & -5.830992 & -2.308301 & 0.496795 \\
\hline 62 & 1 & 0 & -6.638172 & -3.787108 & -0.039086 \\
\hline 63 & 1 & 0 & -5.197469 & -3.881034 & 0.992593 \\
\hline 64 & 6 & 0 & -4.608923 & -4.894636 & -1.466110 \\
\hline 65 & 1 & 0 & -4.011790 & -5.025962 & -2.375523 \\
\hline 66 & 1 & 0 & -4.137987 & -5.463233 & -0.656195 \\
\hline 67 & 1 & 0 & -5.595098 & -5.333962 & -1.651296 \\
\hline 68 & 6 & 0 & -5.695364 & 2.961500 & -1.716741 \\
\hline
\end{tabular}




\begin{tabular}{|c|c|c|c|c|c|}
\hline 69 & 1 & 0 & -6.702993 & 3.388616 & -1.793748 \\
\hline 70 & 1 & 0 & -5.128224 & 3.279521 & -2.599795 \\
\hline 71 & 1 & 0 & -5.773092 & 1.872725 & -1.723532 \\
\hline 72 & 6 & 0 & -5.834107 & 3.091265 & 0.827745 \\
\hline 73 & 1 & 0 & -6.844684 & 3.509317 & 0.747624 \\
\hline 74 & 1 & 0 & -5.928695 & 2.012803 & 0.958741 \\
\hline 75 & 1 & 0 & -5.369415 & 3.498864 & 1.732768 \\
\hline 76 & 6 & 0 & -4.949050 & 5.009677 & -0.493593 \\
\hline 77 & 1 & 0 & -4.473492 & 5.439469 & 0.395294 \\
\hline 78 & 1 & 0 & -4.405074 & 5.355491 & -1.379674 \\
\hline 79 & 1 & 0 & -5.967154 & 5.409418 & -0.554181 \\
\hline 80 & 8 & 0 & 4.406100 & 0.436589 & 0.288414 \\
\hline 81 & 8 & 0 & 4.180280 & -0.218348 & -2.416714 \\
\hline 82 & 8 & 0 & -0.513470 & -0.109175 & 1.961963 \\
\hline 83 & 8 & 0 & -4.529489 & -0.204498 & 1.926772 \\
\hline 84 & 8 & 0 & -5.769427 & -0.025049 & -0.642425 \\
\hline 85 & 6 & 0 & -7.110639 & -0.083234 & -0.224493 \\
\hline 86 & 1 & 0 & -7.611607 & -0.953588 & -0.672792 \\
\hline 87 & 1 & 0 & -7.207836 & -0.159138 & 0.867320 \\
\hline 88 & 1 & 0 & -7.651837 & 0.815990 & -0.553184 \\
\hline 89 & 6 & 0 & -3.778417 & -0.039670 & 3.106695 \\
\hline 90 & 1 & 0 & -4.158229 & -0.722983 & 3.879502 \\
\hline 91 & 1 & 0 & -2.711049 & -0.242159 & 2.954929 \\
\hline 92 & 1 & 0 & -3.879851 & 0.986451 & 3.493494 \\
\hline 93 & 6 & 0 & 0.460541 & 0.212742 & 2.920405 \\
\hline 94 & 1 & 0 & 0.932947 & 1.190187 & 2.736306 \\
\hline 95 & 1 & 0 & -0.003660 & 0.250638 & 3.916328 \\
\hline 96 & 1 & 0 & 1.262835 & -0.540970 & 2.956231 \\
\hline 97 & 6 & 0 & 5.777672 & 0.289668 & 0.483770 \\
\hline 98 & 1 & 0 & 6.218523 & -0.540429 & -0.090072 \\
\hline 99 & 1 & 0 & 6.327868 & 1.206373 & 0.210777 \\
\hline 100 & 1 & 0 & 5.989525 & 0.093866 & 1.545741 \\
\hline 101 & 6 & 0 & 5.483149 & 0.093874 & -2.853678 \\
\hline 102 & 1 & 0 & 6.057944 & -0.819469 & -3.065919 \\
\hline 103 & 1 & 0 & 5.426598 & 0.698919 & -3.768560 \\
\hline 104 & 1 & 0 & 6.027734 & 0.676823 & -2.105309 \\
\hline 105 & 6 & 0 & 2.961592 & 0.921647 & -5.773505 \\
\hline 106 & 6 & 0 & 3.929304 & -0.070345 & -6.413076 \\
\hline
\end{tabular}




$\begin{array}{llllll}107 & 6 & 0 & 3.506053 & -1.537260 & -6.286487 \\ 108 & 6 & 0 & 2.316747 & -1.299082 & -3.995709 \\ 109 & 6 & 0 & 3.366693 & -2.032084 & -4.842693 \\ 110 & 1 & 0 & 1.924211 & 0.688512 & -6.046449 \\ 111 & 1 & 0 & 4.929629 & 0.085933 & -5.989566 \\ 112 & 1 & 0 & 2.546712 & -1.674473 & -6.807556 \\ 113 & 1 & 0 & 1.370212 & -1.212073 & -4.552643 \\ 114 & 1 & 0 & 3.176050 & 1.934861 & -6.120757 \\ 115 & 1 & 0 & 3.998901 & 0.193288 & -7.477377 \\ 116 & 1 & 0 & 4.233011 & -2.167513 & -6.814263 \\ 117 & 1 & 0 & 2.085819 & -1.887667 & -3.106069 \\ 118 & 1 & 0 & 3.085512 & -3.092358 & -4.867330 \\ 119 & 1 & 0 & 4.333010 & -1.983556 & -4.329054 \\ 120 & 6 & 0 & 2.632477 & 0.098086 & -3.481060 \\ 121 & 8 & 0 & 1.998498 & 0.522868 & -2.453310 \\ 122 & 8 & 0 & 3.047061 & 1.056743 & -4.337202\end{array}$


Table S14. Thermochemistry data of TS-1

- Thermochemistry -

Temperature 298.150 Kelvin. Pressure 1.00000 Atm.

Molecular mass: $\quad 838.44225 \mathrm{amu}$.

\begin{tabular}{|c|c|c|}
\hline & (Hartree/Particle) & $(\mathrm{kcal} / \mathrm{mol})$ \\
\hline Zero-point correction $=$ & 1.034442 & \\
\hline Thermal correction to Energy= & 1.098853 & \\
\hline Thermal correction to Enthalpy= & 1.099797 & \\
\hline Thermal correction to Gibbs Free Energy= & 0.936478 & \\
\hline Electronic Energy= & -3217.679000 & -2019124.057 \\
\hline Sum of electronic and zero-point Energies= & -3216.644558 & -2018474.935 \\
\hline Sum of electronic and thermal Energies= & -3216.580147 & -2018434.516 \\
\hline Sum of electronic and thermal Enthalpies $=$ & -3216.579203 & -2018433.924 \\
\hline \multicolumn{2}{|l|}{ Sum of electronic and thermal Free Energies $=$} & -3216.742522 \\
\hline & -2018536.408 & \\
\hline E (Thermal) & $\mathrm{CV}$ & $\mathrm{S}$ \\
\hline KCal/Mol & Cal/Mol-Kelvin & Cal/Mol-Kelvin \\
\hline 689.541 & 242.912 & 343.734 \\
\hline
\end{tabular}


Intermediate II

Table S15. Atomic coordinates of II

$\begin{array}{lcccc}\text { Center } & \text { Atomic } & \text { Atomic } & \text { Coordinates (Angstroms) } \\ \text { Number } & \text { Number } & \text { Type } & X & \text { Y } \\ Z & & & \end{array}$

\begin{tabular}{|c|c|c|c|c|c|}
\hline 1 & 13 & 0 & -0.412348 & -0.049773 & 0.246898 \\
\hline 2 & 7 & 0 & 0.922360 & 1.266719 & -0.463107 \\
\hline 3 & 6 & 0 & 0.385282 & 2.476089 & -0.698703 \\
\hline 4 & 6 & 0 & 2.561732 & 2.756661 & -0.359249 \\
\hline 5 & 6 & 0 & 1.379460 & 3.447111 & -0.650077 \\
\hline 6 & 1 & 0 & 1.258429 & 4.511717 & -0.768084 \\
\hline 7 & 7 & 0 & -1.649845 & 1.305992 & -0.524060 \\
\hline 8 & 6 & 0 & -1.059803 & 2.470485 & -0.847715 \\
\hline 9 & 6 & 0 & -3.241881 & 2.698824 & -1.173357 \\
\hline 10 & 6 & 0 & -2.023285 & 3.380015 & -1.263904 \\
\hline 11 & 1 & 0 & -1.864530 & 4.392666 & -1.597117 \\
\hline 12 & 7 & 0 & 0.877885 & -1.541151 & -0.048142 \\
\hline 13 & 6 & 0 & 0.309937 & -2.758683 & -0.045277 \\
\hline 14 & 6 & 0 & 1.284713 & -3.726410 & 0.171960 \\
\hline 15 & 6 & 0 & 2.485969 & -3.020554 & 0.300520 \\
\hline 16 & 1 & 0 & 1.141074 & -4.791862 & 0.251357 \\
\hline 17 & 7 & 0 & -1.688254 & -1.518983 & -0.191212 \\
\hline 18 & 6 & 0 & -1.132934 & -2.744281 & -0.211449 \\
\hline 19 & 6 & 0 & -3.318131 & -2.983393 & -0.509066 \\
\hline 20 & 6 & 0 & -2.120044 & -3.699253 & -0.411533 \\
\hline 21 & 1 & 0 & -1.989132 & -4.766338 & -0.486690 \\
\hline 22 & 7 & 0 & 2.225668 & -1.683503 & 0.159081 \\
\hline 23 & 7 & 0 & 2.272523 & 1.420995 & -0.257751 \\
\hline 24 & 7 & 0 & -3.000043 & 1.428391 & -0.722359 \\
\hline 25 & 7 & 0 & -3.041267 & -1.648496 & -0.373554 \\
\hline 26 & 13 & 0 & 3.312613 & -0.214139 & -0.552036 \\
\hline 27 & 13 & 0 & -4.127563 & -0.040751 & -0.054455 \\
\hline 28 & 6 & 0 & 3.856002 & -3.621122 & 0.595242 \\
\hline 29 & 6 & 0 & 3.929682 & 3.387000 & -0.118452 \\
\hline 30 & 6 & 0 & -4.696703 & -3.594030 & -0.741304 \\
\hline
\end{tabular}




\begin{tabular}{|c|c|c|c|c|c|}
\hline 31 & 6 & 0 & -4.610547 & 3.270388 & -1.526406 \\
\hline 32 & 6 & 0 & 4.799927 & -3.464292 & -0.619540 \\
\hline 33 & 1 & 0 & 4.977698 & -2.420713 & -0.881659 \\
\hline 34 & 1 & 0 & 5.769357 & -3.924488 & -0.393909 \\
\hline 35 & 1 & 0 & 4.383243 & -3.963395 & -1.501424 \\
\hline 36 & 6 & 0 & 4.457851 & -2.955813 & 1.854640 \\
\hline 37 & 1 & 0 & 5.434318 & -3.402538 & 2.078004 \\
\hline 38 & 1 & 0 & 4.587847 & -1.880925 & 1.714779 \\
\hline 39 & 1 & 0 & 3.807920 & -3.117776 & 2.722635 \\
\hline 40 & 6 & 0 & 3.702085 & -5.131158 & 0.877079 \\
\hline 41 & 1 & 0 & 3.040323 & -5.319087 & 1.729890 \\
\hline 42 & 1 & 0 & 3.309938 & -5.671643 & 0.008135 \\
\hline 43 & 1 & 0 & 4.683102 & -5.556171 & 1.115318 \\
\hline 44 & 6 & 0 & 4.930392 & 2.990251 & -1.225872 \\
\hline 45 & 1 & 0 & 5.095652 & 1.914063 & -1.276008 \\
\hline 46 & 1 & 0 & 4.571349 & 3.308691 & -2.209469 \\
\hline 47 & 1 & 0 & 5.896019 & 3.474406 & -1.034539 \\
\hline 48 & 6 & 0 & 4.454523 & 2.970820 & 1.275562 \\
\hline 49 & 1 & 0 & 3.761002 & 3.292327 & 2.061719 \\
\hline 50 & 1 & 0 & 4.584285 & 1.889093 & 1.345906 \\
\hline 51 & 1 & 0 & 5.421835 & 3.452665 & 1.463376 \\
\hline 52 & 6 & 0 & 3.792345 & 4.924865 & -0.138612 \\
\hline 53 & 1 & 0 & 3.454918 & 5.292581 & -1.114359 \\
\hline 54 & 1 & 0 & 3.095420 & 5.281752 & 0.627937 \\
\hline 55 & 1 & 0 & 4.769771 & 5.375835 & 0.063805 \\
\hline 56 & 6 & 0 & -5.263306 & -3.128189 & -2.102979 \\
\hline 57 & 1 & 0 & -4.597118 & -3.431771 & -2.919387 \\
\hline 58 & 1 & 0 & -6.240154 & -3.596739 & -2.275812 \\
\hline 59 & 1 & 0 & -5.384472 & -2.043937 & -2.132177 \\
\hline 60 & 6 & 0 & -5.652884 & -3.230478 & 0.416839 \\
\hline 61 & 1 & 0 & -5.802667 & -2.154752 & 0.509722 \\
\hline 62 & 1 & 0 & -6.632594 & -3.692621 & 0.246468 \\
\hline 63 & 1 & 0 & -5.264164 & -3.599814 & 1.372421 \\
\hline 64 & 6 & 0 & -4.574851 & -5.132976 & -0.778440 \\
\hline 65 & 1 & 0 & -3.931588 & -5.470939 & -1.598559 \\
\hline 66 & 1 & 0 & -4.180506 & -5.535347 & 0.161567 \\
\hline 67 & 1 & 0 & -5.567393 & -5.568251 & -0.936835 \\
\hline 68 & 6 & 0 & -5.209831 & 2.506707 & -2.730604 \\
\hline
\end{tabular}




\begin{tabular}{|c|c|c|c|c|c|}
\hline 69 & 1 & 0 & -6.183792 & 2.937428 & -2.993646 \\
\hline 70 & 1 & 0 & -4.555657 & 2.598031 & -3.605853 \\
\hline 71 & 1 & 0 & -5.343582 & 1.447675 & -2.501792 \\
\hline 72 & 6 & 0 & -5.551861 & 3.211258 & -0.301997 \\
\hline 73 & 1 & 0 & -6.530524 & 3.629027 & -0.565889 \\
\hline 74 & 1 & 0 & -5.709969 & 2.192985 & 0.055462 \\
\hline 75 & 1 & 0 & -5.145152 & 3.800377 & 0.527935 \\
\hline 76 & 6 & 0 & -4.459186 & 4.753671 & -1.927004 \\
\hline 77 & 1 & 0 & -4.035625 & 5.355279 & -1.114788 \\
\hline 78 & 1 & 0 & -3.826963 & 4.873317 & -2.813927 \\
\hline 79 & 1 & 0 & -5.445607 & 5.164886 & -2.166906 \\
\hline 80 & 8 & 0 & 4.775184 & -0.105720 & 0.336835 \\
\hline 81 & 8 & 0 & 5.227005 & -0.019484 & -3.039345 \\
\hline 82 & 8 & 0 & -0.504154 & 0.210462 & 1.958582 \\
\hline 83 & 8 & 0 & -4.470574 & 0.150440 & 1.614293 \\
\hline 84 & 8 & 0 & -5.542374 & -0.159870 & -1.038103 \\
\hline 85 & 6 & 0 & -6.915417 & -0.078191 & -0.745198 \\
\hline 86 & 1 & 0 & -7.425160 & -1.001725 & -1.055062 \\
\hline 87 & 1 & 0 & -7.109023 & 0.074690 & 0.325531 \\
\hline 88 & 1 & 0 & -7.374033 & 0.754972 & -1.297125 \\
\hline 89 & 6 & 0 & -3.879696 & 0.776946 & 2.728514 \\
\hline 90 & 1 & 0 & -4.346417 & 0.396361 & 3.647472 \\
\hline 91 & 1 & 0 & -2.799790 & 0.591887 & 2.783593 \\
\hline 92 & 1 & 0 & -4.039971 & 1.866487 & 2.702725 \\
\hline 93 & 6 & 0 & 0.478333 & 0.119126 & 2.957630 \\
\hline 94 & 1 & 0 & 1.262089 & 0.884269 & 2.839397 \\
\hline 95 & 1 & 0 & 0.013799 & 0.270717 & 3.941654 \\
\hline 96 & 1 & 0 & 0.973289 & -0.864713 & 2.972311 \\
\hline 97 & 6 & 0 & 6.165179 & -0.170035 & 0.147186 \\
\hline 98 & 1 & 0 & 6.430718 & -0.314260 & -0.906663 \\
\hline 99 & 1 & 0 & 6.636722 & 0.758692 & 0.498873 \\
\hline 100 & 1 & 0 & 6.588749 & -0.999536 & 0.731357 \\
\hline 101 & 6 & 0 & 6.102014 & 0.479263 & -4.041091 \\
\hline 102 & 1 & 0 & 6.276916 & -0.259531 & -4.835653 \\
\hline 103 & 1 & 0 & 5.722531 & 1.406592 & -4.481742 \\
\hline 104 & 1 & 0 & 7.052980 & 0.679613 & -3.540232 \\
\hline 105 & 6 & 0 & 2.055685 & 1.299711 & -4.106693 \\
\hline 106 & 6 & 0 & 1.915814 & 1.325013 & -5.627509 \\
\hline
\end{tabular}




$\begin{array}{llllll}107 & 6 & 0 & 2.179790 & -0.023293 & -6.308915 \\ 108 & 6 & 0 & 3.632477 & -1.195772 & -4.521377 \\ 109 & 6 & 0 & 3.533660 & -0.668505 & -5.964395 \\ 110 & 1 & 0 & 1.437588 & 0.513841 & -3.654569 \\ 111 & 1 & 0 & 2.611516 & 2.081376 & -6.016034 \\ 112 & 1 & 0 & 1.377752 & -0.727544 & -6.041311 \\ 113 & 1 & 0 & 2.713380 & -1.742065 & -4.283248 \\ 114 & 1 & 0 & 1.728254 & 2.255511 & -3.683631 \\ 115 & 1 & 0 & 0.902709 & 1.669557 & -5.879759 \\ 116 & 1 & 0 & 2.115366 & 0.113344 & -7.396713 \\ 117 & 1 & 0 & 4.445951 & -1.926162 & -4.438939 \\ 118 & 1 & 0 & 3.684581 & -1.522881 & -6.637710 \\ 119 & 1 & 0 & 4.342584 & 0.038775 & -6.183046 \\ 120 & 6 & 0 & 3.851864 & -0.169618 & -3.373840 \\ 121 & 8 & 0 & 3.235573 & -0.640880 & -2.248716 \\ 122 & 8 & 0 & 3.419946 & 1.145888 & -3.704352\end{array}$


Table S16. Thermochemistry data of II

- Thermochemistry -

Temperature 298.150 Kelvin. Pressure 1.00000 Atm.

Molecular mass: $\quad 838.44225 \mathrm{amu}$.

\begin{tabular}{|c|c|c|}
\hline & (Hartree/Particle) & $(\mathrm{kcal} / \mathrm{mol})$ \\
\hline Zero-point correction $=$ & 1.036106 & \\
\hline Thermal correction to Energy= & 1.100826 & \\
\hline Thermal correction to Enthalpy= & 1.10177 & \\
\hline Thermal correction to Gibbs Free Energy= & 0.937489 & \\
\hline Electronic Energy= & -3217.706835 & -2019141.523 \\
\hline Sum of electronic and zero-point Energies= & -3216.670729 & -2018491.357 \\
\hline Sum of electronic and thermal Energies $=$ & -3216.606009 & -2018450.745 \\
\hline Sum of electronic and thermal Enthalpies $=$ & -3216.605065 & -2018450.152 \\
\hline \multicolumn{2}{|l|}{ Sum of electronic and thermal Free Energies $=$} & -3216.769346 \\
\hline & -2018553.240 & \\
\hline E (Thermal) & $\mathrm{CV}$ & $\mathrm{S}$ \\
\hline KCal/Mol & Cal/Mol-Kelvin & Cal/Mol-Kelvin \\
\hline 690.779 & 243.317 & 345.759 \\
\hline
\end{tabular}


Transition state 2

Table S17. Atomic coordinates of TS2

$\begin{array}{lcccc}\text { Center } & \text { Atomic } & \text { Atomic } & \text { Coordinates (Angstroms) } \\ \text { Number } & \text { Number } & \text { Type } & X & \text { Y } \\ Z & & & \end{array}$

\begin{tabular}{|c|c|c|c|c|c|}
\hline 1 & 13 & 0 & -0.535254 & -0.130024 & 0.226644 \\
\hline 2 & 7 & 0 & 0.785135 & 1.110424 & -0.629566 \\
\hline 3 & 6 & 0 & 0.255489 & 2.311954 & -0.912014 \\
\hline 4 & 6 & 0 & 2.443358 & 2.568091 & -0.674433 \\
\hline 5 & 6 & 0 & 1.264798 & 3.267168 & -0.959683 \\
\hline 6 & 1 & 0 & 1.155384 & 4.325766 & -1.132066 \\
\hline 7 & 7 & 0 & -1.792262 & 1.196363 & -0.593459 \\
\hline 8 & 6 & 0 & -1.192235 & 2.324270 & -1.010202 \\
\hline 9 & 6 & 0 & -3.377632 & 2.579725 & -1.283982 \\
\hline 10 & 6 & 0 & -2.150356 & 3.226105 & -1.456286 \\
\hline 11 & 1 & 0 & -1.981140 & 4.209980 & -1.862651 \\
\hline 12 & 7 & 0 & 0.678021 & -1.701186 & 0.051392 \\
\hline 13 & 6 & 0 & 0.055360 & -2.880128 & 0.223528 \\
\hline 14 & 6 & 0 & 0.997151 & -3.873889 & 0.457032 \\
\hline 15 & 6 & 0 & 2.234491 & -3.219929 & 0.414394 \\
\hline 16 & 1 & 0 & 0.812499 & -4.918652 & 0.648496 \\
\hline 17 & 7 & 0 & -1.900911 & -1.579443 & 0.032647 \\
\hline 18 & 6 & 0 & -1.392328 & -2.817736 & 0.153548 \\
\hline 19 & 6 & 0 & -3.601099 & -2.999813 & 0.060867 \\
\hline 20 & 6 & 0 & -2.424829 & -3.746680 & 0.172114 \\
\hline 21 & 1 & 0 & -2.335761 & -4.817918 & 0.249776 \\
\hline 22 & 7 & 0 & 2.028897 & -1.894249 & 0.151027 \\
\hline 23 & 7 & 0 & 2.142087 & 1.242552 & -0.501380 \\
\hline 24 & 7 & 0 & -3.146523 & 1.337005 & -0.753942 \\
\hline 25 & 7 & 0 & -3.268834 & -1.672760 & -0.024565 \\
\hline 26 & 13 & 0 & 3.130631 & -0.486261 & -0.795803 \\
\hline 27 & 13 & 0 & -4.292152 & -0.005073 & 0.107887 \\
\hline 28 & 6 & 0 & 3.588080 & -3.859472 & 0.697597 \\
\hline 29 & 6 & 0 & 3.814857 & 3.195386 & -0.450820 \\
\hline 30 & 6 & 0 & -5.014084 & -3.574429 & 0.024286 \\
\hline
\end{tabular}




\begin{tabular}{|c|c|c|c|c|c|}
\hline 31 & 6 & 0 & -4.743818 & 3.153971 & -1.643876 \\
\hline 32 & 6 & 0 & 4.566485 & -3.640890 & -0.473709 \\
\hline 33 & 1 & 0 & 4.815319 & -2.585643 & -0.583077 \\
\hline 34 & 1 & 0 & 5.499908 & -4.186160 & -0.288423 \\
\hline 35 & 1 & 0 & 4.140057 & -3.993100 & -1.419296 \\
\hline 36 & 6 & 0 & 4.163232 & -3.255315 & 2.000344 \\
\hline 37 & 1 & 0 & 5.155182 & -3.676351 & 2.207414 \\
\hline 38 & 1 & 0 & 4.246630 & -2.170203 & 1.916133 \\
\hline 39 & 1 & 0 & 3.512499 & -3.487550 & 2.851411 \\
\hline 40 & 6 & 0 & 3.415924 & -5.379807 & 0.894807 \\
\hline 41 & 1 & 0 & 2.755338 & -5.607599 & 1.738346 \\
\hline 42 & 1 & 0 & 3.011018 & -5.863400 & -0.001732 \\
\hline 43 & 1 & 0 & 4.391410 & -5.831048 & 1.107983 \\
\hline 44 & 6 & 0 & 4.865028 & 2.629097 & -1.424945 \\
\hline 45 & 1 & 0 & 4.980825 & 1.553688 & -1.299612 \\
\hline 46 & 1 & 0 & 4.580707 & 2.826462 & -2.465417 \\
\hline 47 & 1 & 0 & 5.837987 & 3.101679 & -1.244294 \\
\hline 48 & 6 & 0 & 4.242009 & 2.936066 & 1.013369 \\
\hline 49 & 1 & 0 & 3.533309 & 3.403384 & 1.706894 \\
\hline 50 & 1 & 0 & 4.274013 & 1.865363 & 1.223599 \\
\hline 51 & 1 & 0 & 5.233060 & 3.368768 & 1.199213 \\
\hline 52 & 6 & 0 & 3.735083 & 4.719794 & -0.674802 \\
\hline 53 & 1 & 0 & 3.433845 & 4.966999 & -1.699541 \\
\hline 54 & 1 & 0 & 3.030595 & 5.194722 & 0.016388 \\
\hline 55 & 1 & 0 & 4.721195 & 5.164366 & -0.500588 \\
\hline 56 & 6 & 0 & -5.657696 & -3.312883 & -1.357774 \\
\hline 57 & 1 & 0 & -5.066049 & -3.785775 & -2.150876 \\
\hline 58 & 1 & 0 & -6.664292 & -3.748403 & -1.385111 \\
\hline 59 & 1 & 0 & -5.730280 & -2.243509 & -1.563884 \\
\hline 60 & 6 & 0 & -5.875662 & -2.984346 & 1.163158 \\
\hline 61 & 1 & 0 & -5.986254 & -1.902457 & 1.089439 \\
\hline 62 & 1 & 0 & -6.878642 & -3.426958 & 1.134682 \\
\hline 63 & 1 & 0 & -5.433229 & -3.208367 & 2.140271 \\
\hline 64 & 6 & 0 & -4.949954 & -5.102766 & 0.237328 \\
\hline 65 & 1 & 0 & -4.382328 & -5.601337 & -0.556280 \\
\hline 66 & 1 & 0 & -4.499222 & -5.362522 & 1.201914 \\
\hline 67 & 1 & 0 & -5.966161 & -5.511518 & 0.223292 \\
\hline 68 & 6 & 0 & -5.402412 & 2.300525 & -2.752868 \\
\hline
\end{tabular}




\begin{tabular}{|c|c|c|c|c|c|}
\hline 69 & 1 & 0 & -6.376194 & 2.729263 & -3.020292 \\
\hline 70 & 1 & 0 & -4.777841 & 2.298378 & -3.654283 \\
\hline 71 & 1 & 0 & -5.549261 & 1.269207 & -2.426788 \\
\hline 72 & 6 & 0 & -5.644593 & 3.238544 & -0.390542 \\
\hline 73 & 1 & 0 & -6.623635 & 3.649833 & -0.664054 \\
\hline 74 & 1 & 0 & -5.808612 & 2.266982 & 0.076942 \\
\hline 75 & 1 & 0 & -5.197794 & 3.898994 & 0.361478 \\
\hline 76 & 6 & 0 & -4.570297 & 4.588440 & -2.188038 \\
\hline 77 & 1 & 0 & -4.108001 & 5.253008 & -1.449409 \\
\hline 78 & 1 & 0 & -3.963262 & 4.607419 & -3.100148 \\
\hline 79 & 1 & 0 & -5.554172 & 5.000584 & -2.437223 \\
\hline 80 & 8 & 0 & 4.391700 & -0.323453 & 0.414140 \\
\hline 81 & 8 & 0 & 2.815945 & -2.396434 & -3.632092 \\
\hline 82 & 8 & 0 & -0.566275 & 0.292896 & 1.912404 \\
\hline 83 & 8 & 0 & -4.619721 & 0.419490 & 1.738693 \\
\hline 84 & 8 & 0 & -5.738741 & -0.195658 & -0.821552 \\
\hline 85 & 6 & 0 & -7.095847 & -0.033357 & -0.492343 \\
\hline 86 & 1 & 0 & -7.642147 & -0.973849 & -0.655282 \\
\hline 87 & 1 & 0 & -7.242710 & 0.267839 & 0.554164 \\
\hline 88 & 1 & 0 & -7.555183 & 0.732289 & -1.134517 \\
\hline 89 & 6 & 0 & -3.907795 & 0.983836 & 2.815292 \\
\hline 90 & 1 & 0 & -4.291418 & 0.576351 & 3.761484 \\
\hline 91 & 1 & 0 & -2.830842 & 0.779702 & 2.762182 \\
\hline 92 & 1 & 0 & -4.048530 & 2.075776 & 2.844930 \\
\hline 93 & 6 & 0 & 0.467090 & 0.322245 & 2.862285 \\
\hline 94 & 1 & 0 & 1.164602 & 1.156936 & 2.687972 \\
\hline 95 & 1 & 0 & 0.038294 & 0.452830 & 3.866003 \\
\hline 96 & 1 & 0 & 1.059277 & -0.606316 & 2.871708 \\
\hline 97 & 6 & 0 & 5.762326 & -0.316555 & 0.656231 \\
\hline 98 & 1 & 0 & 6.279409 & -1.191585 & 0.229590 \\
\hline 99 & 1 & 0 & 6.258667 & 0.582637 & 0.255320 \\
\hline 100 & 1 & 0 & 5.952826 & -0.330821 & 1.740042 \\
\hline 101 & 6 & 0 & 2.960808 & -2.906681 & -4.956744 \\
\hline 102 & 1 & 0 & 2.309862 & -2.387404 & -5.666488 \\
\hline 103 & 1 & 0 & 3.997663 & -2.860760 & -5.303257 \\
\hline 104 & 1 & 0 & 2.653421 & -3.952388 & -4.894427 \\
\hline 105 & 6 & 0 & 5.618331 & -0.812709 & -2.636379 \\
\hline 106 & 6 & 0 & 5.922755 & -0.777714 & -4.134013 \\
\hline
\end{tabular}




$\begin{array}{rrrrrr}107 & 6 & 0 & 5.279314 & 0.397968 & -4.892462 \\ 108 & 6 & 0 & 2.723921 & -0.014899 & -4.438109 \\ 109 & 6 & 0 & 3.873665 & 0.120841 & -5.451800 \\ 110 & 1 & 0 & 6.160213 & 0.008797 & -2.150389 \\ 111 & 1 & 0 & 5.622815 & -1.726109 & -4.598022 \\ 112 & 1 & 0 & 5.256369 & 1.286270 & -4.247726 \\ 113 & 1 & 0 & 2.609023 & 0.928150 & -3.897836 \\ 114 & 1 & 0 & 5.997066 & -1.751865 & -2.210786 \\ 115 & 1 & 0 & 7.015515 & -0.730762 & -4.232776 \\ 116 & 1 & 0 & 5.918121 & 0.657667 & -5.746379 \\ 117 & 1 & 0 & 1.780173 & -0.159950 & -4.986112 \\ 118 & 1 & 0 & 3.595525 & 0.960091 & -6.103174 \\ 119 & 1 & 0 & 3.924872 & -0.751395 & -6.112508 \\ 120 & 6 & 0 & 2.714762 & -1.074318 & -3.338683 \\ 121 & 8 & 0 & 1.983221 & -0.837845 & -2.314408 \\ 122 & 8 & 0 & 4.235822 & -0.662060 & -2.356195 \\ & 1 & & & & \end{array}$




\section{Table S18. Thermochemistry data of TS2}

- Thermochemistry -

Temperature 298.150 Kelvin. Pressure 1.00000 Atm.

Molecular mass: $\quad 838.44225$ amu.

\begin{tabular}{|c|c|c|}
\hline & (Hartree/Particle) & $(\mathrm{kcal} / \mathrm{mol})$ \\
\hline Zero-point correction $=$ & 1.034309 & \\
\hline Thermal correction to Energy= & 1.098878 & \\
\hline Thermal correction to Enthalpy= & 1.099823 & \\
\hline Thermal correction to Gibbs Free Energy= & 0.935732 & \\
\hline Electronic Energy= & -3217.670734 & -2019118.870 \\
\hline Sum of electronic and zero-point Energies= & -3216.636425 & -2018469.831 \\
\hline Sum of electronic and thermal Energies= & -3216.571856 & -2018429.313 \\
\hline Sum of electronic and thermal Enthalpies $=$ & -3216.570911 & -2018428.720 \\
\hline \multicolumn{2}{|l|}{ Sum of electronic and thermal Free Energies= } & -3216.735002 \\
\hline & -2018531.689 & \\
\hline E (Thermal) & $\mathrm{CV}$ & $\mathrm{S}$ \\
\hline $\mathrm{KCal} / \mathrm{Mol}$ & Cal/Mol-Kelvin & Cal/Mol-Kelvin \\
\hline 689.557 & 243.032 & 345.359 \\
\hline
\end{tabular}


Intermediate III

Table S19. Atomic coordinates of III

$\begin{array}{lcccc}\text { Center } & \text { Atomic } & \text { Atomic } & \text { Coordinates (Angstroms) } \\ \text { Number } & \text { Number } & \text { Type } & X & \text { Y } \\ Z & & & \end{array}$

\begin{tabular}{|c|c|c|c|c|c|}
\hline 1 & 13 & 0 & -0.110429 & 0.101438 & -1.004315 \\
\hline 2 & 7 & 0 & 1.237570 & -1.339807 & -0.695990 \\
\hline 3 & 6 & 0 & 0.702406 & -2.569288 & -0.636917 \\
\hline 4 & 6 & 0 & 2.890319 & -2.791388 & -0.948344 \\
\hline 5 & 6 & 0 & 1.708425 & -3.520454 & -0.784443 \\
\hline 6 & 1 & 0 & 1.595228 & -4.592328 & -0.802526 \\
\hline 7 & 7 & 0 & -1.343664 & -1.398177 & -0.577773 \\
\hline 8 & 6 & 0 & -0.743597 & -2.596031 & -0.491322 \\
\hline 9 & 6 & 0 & -2.924002 & -2.899725 & -0.206678 \\
\hline 10 & 6 & 0 & -1.701256 & -3.576551 & -0.255753 \\
\hline 11 & 1 & 0 & -1.536056 & -4.635224 & -0.137768 \\
\hline 12 & 7 & 0 & 1.188416 & 1.513786 & -0.451769 \\
\hline 13 & 6 & 0 & 0.609230 & 2.707028 & -0.245109 \\
\hline 14 & 6 & 0 & 1.569891 & 3.711555 & -0.336923 \\
\hline 15 & 6 & 0 & 2.769320 & 3.055766 & -0.627234 \\
\hline 16 & 1 & 0 & 1.412978 & 4.774107 & -0.244106 \\
\hline 17 & 7 & 0 & -1.392784 & 1.472111 & -0.370915 \\
\hline 18 & 6 & 0 & -0.834132 & 2.656958 & -0.077553 \\
\hline 19 & 6 & 0 & -3.013901 & 2.815525 & 0.301569 \\
\hline 20 & 6 & 0 & -1.820072 & 3.540515 & 0.347716 \\
\hline 21 & 1 & 0 & -1.688701 & 4.562770 & 0.663119 \\
\hline 22 & 7 & 0 & 2.524730 & 1.706291 & -0.687015 \\
\hline 23 & 7 & 0 & 2.588378 & -1.454068 & -0.886047 \\
\hline 24 & 7 & 0 & -2.688967 & -1.562292 & -0.399429 \\
\hline 25 & 7 & 0 & -2.736802 & 1.545790 & -0.135713 \\
\hline 26 & 13 & 0 & 3.671352 & 0.131755 & -0.481346 \\
\hline 27 & 13 & 0 & -3.814195 & 0.017542 & -0.742670 \\
\hline 28 & 6 & 0 & 4.111283 & 3.718429 & -0.922846 \\
\hline 29 & 6 & 0 & 4.271039 & -3.377876 & -1.227948 \\
\hline 30 & 6 & 0 & -4.393784 & 3.341257 & 0.679017 \\
\hline
\end{tabular}




\begin{tabular}{|c|c|c|c|c|c|}
\hline 31 & 6 & 0 & -4.285881 & -3.545483 & 0.027961 \\
\hline 32 & 6 & 0 & 5.188622 & 3.296266 & 0.100154 \\
\hline 33 & 1 & 0 & 5.355208 & 2.217523 & 0.112423 \\
\hline 34 & 1 & 0 & 6.142815 & 3.776876 & -0.146184 \\
\hline 35 & 1 & 0 & 4.906174 & 3.607695 & 1.112082 \\
\hline 36 & 6 & 0 & 4.554364 & 3.360572 & -2.361452 \\
\hline 37 & 1 & 0 & 5.503014 & 3.860965 & -2.591838 \\
\hline 38 & 1 & 0 & 4.687106 & 2.283120 & -2.480973 \\
\hline 39 & 1 & 0 & 3.808429 & 3.701447 & -3.088813 \\
\hline 40 & 6 & 0 & 3.957037 & 5.251594 & -0.833379 \\
\hline 41 & 1 & 0 & 3.226806 & 5.627743 & -1.558110 \\
\hline 42 & 1 & 0 & 3.649204 & 5.573728 & 0.167997 \\
\hline 43 & 1 & 0 & 4.919425 & 5.725749 & -1.054606 \\
\hline 44 & 6 & 0 & 5.281083 & -3.011514 & -0.117549 \\
\hline 45 & 1 & 0 & 5.427009 & -1.936200 & -0.013171 \\
\hline 46 & 1 & 0 & 4.946473 & -3.392997 & 0.853800 \\
\hline 47 & 1 & 0 & 6.253716 & -3.464789 & -0.344170 \\
\hline 48 & 6 & 0 & 4.768131 & -2.887330 & -2.608540 \\
\hline 49 & 1 & 0 & 4.081247 & -3.208934 & -3.400194 \\
\hline 50 & 1 & 0 & 4.845153 & -1.798727 & -2.639974 \\
\hline 51 & 1 & 0 & 5.753674 & -3.319307 & -2.821610 \\
\hline 52 & 6 & 0 & 4.171754 & -4.917840 & -1.275299 \\
\hline 53 & 1 & 0 & 3.834951 & -5.335009 & -0.319258 \\
\hline 54 & 1 & 0 & 3.489093 & -5.257318 & -2.061972 \\
\hline 55 & 1 & 0 & 5.160658 & -5.336771 & -1.490767 \\
\hline 56 & 6 & 0 & -4.939984 & 2.568157 & 1.902137 \\
\hline 57 & 1 & 0 & -4.263505 & 2.681709 & 2.757566 \\
\hline 58 & 1 & 0 & -5.918699 & 2.972475 & 2.189527 \\
\hline 59 & 1 & 0 & -5.048940 & 1.504453 & 1.681947 \\
\hline 60 & 6 & 0 & -5.358259 & 3.238486 & -0.524434 \\
\hline 61 & 1 & 0 & -5.479149 & 2.213569 & -0.877823 \\
\hline 62 & 1 & 0 & -6.348533 & 3.614977 & -0.241457 \\
\hline 63 & 1 & 0 & -4.993965 & 3.841715 & -1.363925 \\
\hline 64 & 6 & 0 & -4.287333 & 4.832277 & 1.064851 \\
\hline 65 & 1 & 0 & -3.641320 & 4.980795 & 1.937374 \\
\hline 66 & 1 & 0 & -3.900636 & 5.440857 & 0.239580 \\
\hline 67 & 1 & 0 & -5.282723 & 5.211441 & 1.320988 \\
\hline 68 & 6 & 0 & -4.875075 & -3.071134 & 1.377008 \\
\hline
\end{tabular}




\begin{tabular}{|c|c|c|c|c|c|}
\hline 69 & 1 & 0 & -5.842048 & -3.559298 & 1.551895 \\
\hline 70 & 1 & 0 & -4.207747 & -3.345923 & 2.203314 \\
\hline 71 & 1 & 0 & -5.020712 & -1.989369 & 1.384983 \\
\hline 72 & 6 & 0 & -5.245771 & -3.229700 & -1.140846 \\
\hline 73 & 1 & 0 & -6.211225 & -3.721252 & -0.970473 \\
\hline 74 & 1 & 0 & -5.429003 & -2.160779 & -1.249725 \\
\hline 75 & 1 & 0 & -4.838358 & -3.599176 & -2.088459 \\
\hline 76 & 6 & 0 & -4.121015 & -5.079428 & 0.093190 \\
\hline 77 & 1 & 0 & -3.702312 & -5.485206 & -0.834651 \\
\hline 78 & 1 & 0 & -3.478560 & -5.385439 & 0.926638 \\
\hline 79 & 1 & 0 & -5.102555 & -5.540960 & 0.246610 \\
\hline 80 & 8 & 0 & 4.953985 & 0.209189 & -1.639086 \\
\hline 81 & 8 & 0 & -1.105507 & 0.243804 & 3.852903 \\
\hline 82 & 8 & 0 & -0.207836 & 0.248251 & -2.738436 \\
\hline 83 & 8 & 0 & -4.192582 & 0.155512 & -2.411309 \\
\hline 84 & 8 & 0 & -5.217288 & -0.135002 & 0.257865 \\
\hline 85 & 6 & 0 & -6.592446 & -0.087601 & -0.031407 \\
\hline 86 & 1 & 0 & -7.068674 & 0.742775 & 0.510137 \\
\hline 87 & 1 & 0 & -6.793156 & 0.045422 & -1.103475 \\
\hline 88 & 1 & 0 & -7.081091 & -1.017977 & 0.292916 \\
\hline 89 & 6 & 0 & -3.589384 & 0.742950 & -3.541444 \\
\hline 90 & 1 & 0 & -3.743595 & 1.833595 & -3.551168 \\
\hline 91 & 1 & 0 & -2.509962 & 0.551296 & -3.583019 \\
\hline 92 & 1 & 0 & -4.052160 & 0.335663 & -4.451083 \\
\hline 93 & 6 & 0 & 0.796645 & 0.206713 & -3.715090 \\
\hline 94 & 1 & 0 & 1.348746 & -0.747319 & -3.711869 \\
\hline 95 & 1 & 0 & 0.346686 & 0.321925 & -4.711477 \\
\hline 96 & 1 & 0 & 1.534742 & 1.016520 & -3.592356 \\
\hline 97 & 6 & 0 & 6.353068 & 0.235425 & -1.516039 \\
\hline 98 & 1 & 0 & 6.688539 & 0.187024 & -0.469657 \\
\hline 99 & 1 & 0 & 6.799675 & -0.614928 & -2.051298 \\
\hline 100 & 1 & 0 & 6.759440 & 1.156587 & -1.958426 \\
\hline 101 & 6 & 0 & -1.516387 & -0.225277 & 5.141113 \\
\hline 102 & 1 & 0 & -2.218943 & -1.060960 & 5.052972 \\
\hline 103 & 1 & 0 & -0.666157 & -0.519139 & 5.763520 \\
\hline 104 & 1 & 0 & -2.026121 & 0.619861 & 5.606330 \\
\hline 105 & 6 & 0 & 4.153156 & 0.547572 & 2.422671 \\
\hline 106 & 6 & 0 & 2.889373 & 0.183257 & 3.209735 \\
\hline
\end{tabular}




\begin{tabular}{lrrrrr}
107 & 6 & 0 & 2.744407 & -1.326198 & 3.444549 \\
108 & 6 & 0 & 0.175707 & -1.854243 & 3.490504 \\
109 & 6 & 0 & 1.515935 & -1.758092 & 4.267722 \\
110 & 1 & 0 & 5.038542 & 0.211281 & 2.986846 \\
111 & 1 & 0 & 2.022408 & 0.576254 & 2.670468 \\
112 & 1 & 0 & 2.739172 & -1.843107 & 2.477774 \\
113 & 1 & 0 & 0.325438 & -2.464640 & 2.595890 \\
114 & 1 & 0 & 4.220602 & 1.645114 & 2.357780 \\
115 & 1 & 0 & 2.929956 & 0.703732 & 4.179452 \\
116 & 1 & 0 & 3.642403 & -1.678002 & 3.972811 \\
117 & 1 & 0 & -0.562272 & -2.366261 & 4.117781 \\
118 & 1 & 0 & 1.700582 & -2.760556 & 4.673945 \\
119 & 1 & 0 & 1.403752 & -1.095982 & 5.137247 \\
120 & 6 & 0 & -0.381129 & -0.527054 & 3.005293 \\
121 & 8 & 0 & -0.182660 & -0.097804 & 1.888262 \\
122 & 8 & 0 & 4.195366 & -0.038537 & 1.151390 \\
\hline
\end{tabular}


Table S20. Thermochemistry data of III

- Thermochemistry -

Temperature 298.150 Kelvin. Pressure 1.00000 Atm.

Molecular mass: $\quad 838.44225 \mathrm{amu}$.

\begin{tabular}{|c|c|c|}
\hline & (Hartree/Particle) & $(\mathrm{kcal} / \mathrm{mol})$ \\
\hline Zero-point correction $=$ & 1.034231 & \\
\hline Thermal correction to Energy= & 1.100489 & \\
\hline Thermal correction to Enthalpy= & 1.101434 & \\
\hline Thermal correction to Gibbs Free Energy= & 0.931718 & \\
\hline Electronic Energy= & -3217.708875 & -2019142.804 \\
\hline Sum of electronic and zero-point Energies= & -3216.674644 & -2018493.814 \\
\hline Sum of electronic and thermal Energies $=$ & -3216.608386 & -2018452.236 \\
\hline Sum of electronic and thermal Enthalpies $=$ & -3216.607441 & -2018451.643 \\
\hline \multicolumn{2}{|l|}{ Sum of electronic and thermal Free Energies $=$} & -3216.777157 \\
\hline & -2018558.142 & \\
\hline E (Thermal) & $\mathrm{CV}$ & $\mathrm{S}$ \\
\hline KCal/Mol & Cal/Mol-Kelvin & Cal/Mol-Kelvin \\
\hline 690.568 & 245.405 & 357.196 \\
\hline
\end{tabular}


Intermediate IV

Table S21. Atomic coordinates of IV

$\begin{array}{lcccc}\text { Center } & \text { Atomic } & \text { Atomic } & \text { Coordinates (Angstroms) } \\ \text { Number } & \text { Number } & \text { Type } & X & Y \\ Z & & & \end{array}$

\begin{tabular}{|c|c|c|c|c|c|}
\hline 1 & 13 & 0 & -0.236158 & 0.078554 & 0.592378 \\
\hline 2 & 7 & 0 & 1.059507 & 1.523607 & 0.101114 \\
\hline 3 & 6 & 0 & 0.501914 & 2.743926 & 0.018790 \\
\hline 4 & 6 & 0 & 2.694276 & 3.012533 & 0.236260 \\
\hline 5 & 6 & 0 & 1.492813 & 3.715672 & 0.095643 \\
\hline 6 & 1 & 0 & 1.360780 & 4.785328 & 0.078443 \\
\hline 7 & 7 & 0 & -1.514489 & 1.527943 & 0.088863 \\
\hline 8 & 6 & 0 & -0.947587 & 2.737542 & -0.066087 \\
\hline 9 & 6 & 0 & -3.144361 & 2.989257 & -0.242236 \\
\hline 10 & 6 & 0 & -1.934269 & 3.690365 & -0.279831 \\
\hline 11 & 1 & 0 & -1.796330 & 4.746304 & -0.445482 \\
\hline 12 & 7 & 0 & 1.059635 & -1.301551 & -0.039489 \\
\hline 13 & 6 & 0 & 0.503790 & -2.507300 & -0.258143 \\
\hline 14 & 6 & 0 & 1.487197 & -3.487600 & -0.240675 \\
\hline 15 & 6 & 0 & 2.684636 & -2.810207 & 0.010207 \\
\hline 16 & 1 & 0 & 1.359295 & -4.546772 & -0.389011 \\
\hline 17 & 7 & 0 & -1.511204 & -1.303418 & -0.069940 \\
\hline 18 & 6 & 0 & -0.943502 & -2.484454 & -0.373674 \\
\hline 19 & 6 & 0 & -3.135770 & -2.695861 & -0.636301 \\
\hline 20 & 6 & 0 & -1.927856 & -3.393622 & -0.738536 \\
\hline 21 & 1 & 0 & -1.786973 & -4.417007 & -1.045726 \\
\hline 22 & 7 & 0 & 2.413010 & -1.470752 & 0.125576 \\
\hline 23 & 7 & 0 & 2.417897 & 1.670234 & 0.233465 \\
\hline 24 & 7 & 0 & -2.874284 & 1.664804 & -0.019867 \\
\hline 25 & 7 & 0 & -2.868673 & -1.415100 & -0.230511 \\
\hline 26 & 13 & 0 & 3.511981 & 0.106433 & -0.234902 \\
\hline 27 & 13 & 0 & -3.954067 & 0.084459 & 0.434464 \\
\hline 28 & 6 & 0 & 4.055218 & -3.451376 & 0.204939 \\
\hline 29 & 6 & 0 & 4.076916 & 3.630003 & 0.421276 \\
\hline 30 & 6 & 0 & -4.519531 & -3.263682 & -0.932413 \\
\hline
\end{tabular}




\begin{tabular}{|c|c|c|c|c|c|}
\hline 31 & 6 & 0 & -4.528425 & 3.604385 & -0.425648 \\
\hline 32 & 6 & 0 & 5.040168 & -3.038155 & -0.912418 \\
\hline 33 & 1 & 0 & 5.180431 & -1.958171 & -0.975762 \\
\hline 34 & 1 & 0 & 6.019147 & -3.493401 & -0.719383 \\
\hline 35 & 1 & 0 & 4.684526 & -3.392526 & -1.884611 \\
\hline 36 & 6 & 0 & 4.606522 & -3.065285 & 1.597326 \\
\hline 37 & 1 & 0 & 5.576414 & -3.551855 & 1.758008 \\
\hline 38 & 1 & 0 & 4.737842 & -1.984853 & 1.690928 \\
\hline 39 & 1 & 0 & 3.926712 & -3.402059 & 2.389079 \\
\hline 40 & 6 & 0 & 3.909285 & -4.987294 & 0.140211 \\
\hline 41 & 1 & 0 & 3.232819 & -5.363660 & 0.916198 \\
\hline 42 & 1 & 0 & 3.543307 & -5.310709 & -0.839713 \\
\hline 43 & 1 & 0 & 4.889819 & -5.448359 & 0.303046 \\
\hline 44 & 6 & 0 & 5.010758 & 3.274153 & -0.757850 \\
\hline 45 & 1 & 0 & 5.183981 & 2.201460 & -0.848193 \\
\hline 46 & 1 & 0 & 4.590144 & 3.624282 & -1.707276 \\
\hline 47 & 1 & 0 & 5.982636 & 3.761814 & -0.615998 \\
\hline 48 & 6 & 0 & 4.681136 & 3.157673 & 1.764466 \\
\hline 49 & 1 & 0 & 4.042377 & 3.461876 & 2.602033 \\
\hline 50 & 1 & 0 & 4.793911 & 2.071898 & 1.789901 \\
\hline 51 & 1 & 0 & 5.666391 & 3.617868 & 1.908674 \\
\hline 52 & 6 & 0 & 3.947332 & 5.167708 & 0.467058 \\
\hline 53 & 1 & 0 & 3.544666 & 5.572448 & -0.468569 \\
\hline 54 & 1 & 0 & 3.306128 & 5.496783 & 1.292249 \\
\hline 55 & 1 & 0 & 4.938176 & 5.608781 & 0.619955 \\
\hline 56 & 6 & 0 & -5.155749 & -2.519600 & -2.129583 \\
\hline 57 & 1 & 0 & -4.528582 & -2.625200 & -3.022867 \\
\hline 58 & 1 & 0 & -6.137248 & -2.954270 & -2.355886 \\
\hline 59 & 1 & 0 & -5.283379 & -1.457178 & -1.913409 \\
\hline 60 & 6 & 0 & -5.417822 & -3.173542 & 0.322143 \\
\hline 61 & 1 & 0 & -5.556951 & -2.146560 & 0.662024 \\
\hline 62 & 1 & 0 & -6.407520 & -3.590348 & 0.100881 \\
\hline 63 & 1 & 0 & -4.986228 & -3.747956 & 1.149807 \\
\hline 64 & 6 & 0 & -4.391737 & -4.755557 & -1.308497 \\
\hline 65 & 1 & 0 & -3.787792 & -4.896955 & -2.211725 \\
\hline 66 & 1 & 0 & -3.947144 & -5.344655 & -0.498437 \\
\hline 67 & 1 & 0 & -5.388333 & -5.163434 & -1.509150 \\
\hline 68 & 6 & 0 & -5.182608 & 3.071911 & -1.722086 \\
\hline
\end{tabular}




\begin{tabular}{|c|c|c|c|c|c|}
\hline 69 & 1 & 0 & -6.163493 & 3.543474 & -1.860686 \\
\hline 70 & 1 & 0 & -4.565149 & 3.320797 & -2.593528 \\
\hline 71 & 1 & 0 & -5.316547 & 1.989376 & -1.681962 \\
\hline 72 & 6 & 0 & -5.416724 & 3.317256 & 0.805596 \\
\hline 73 & 1 & 0 & -6.396337 & 3.792153 & 0.673666 \\
\hline 74 & 1 & 0 & -5.581179 & 2.250500 & 0.957988 \\
\hline 75 & 1 & 0 & -4.962393 & 3.721911 & 1.716810 \\
\hline 76 & 6 & 0 & -4.393236 & 5.137193 & -0.555514 \\
\hline 77 & 1 & 0 & -3.934415 & 5.584568 & 0.333514 \\
\hline 78 & 1 & 0 & -3.800917 & 5.420952 & -1.432641 \\
\hline 79 & 1 & 0 & -5.389348 & 5.577023 & -0.674206 \\
\hline 80 & 8 & 0 & 4.921079 & 0.090637 & 0.755401 \\
\hline 81 & 8 & 0 & 4.074669 & -5.058033 & -4.808352 \\
\hline 82 & 8 & 0 & -0.283222 & -0.008214 & 2.322413 \\
\hline 83 & 8 & 0 & -4.211911 & -0.031918 & 2.125211 \\
\hline 84 & 8 & 0 & -5.421127 & 0.174563 & -0.474686 \\
\hline 85 & 6 & 0 & -6.773552 & 0.115370 & -0.093058 \\
\hline 86 & 1 & 0 & -7.299225 & 1.025377 & -0.416299 \\
\hline 87 & 1 & 0 & -7.268341 & -0.741484 & -0.572916 \\
\hline 88 & 1 & 0 & -6.898983 & 0.017274 & 0.994088 \\
\hline 89 & 6 & 0 & -3.573233 & -0.617132 & 3.235602 \\
\hline 90 & 1 & 0 & -3.720541 & -1.708748 & 3.249402 \\
\hline 91 & 1 & 0 & -2.494950 & -0.417043 & 3.243522 \\
\hline 92 & 1 & 0 & -4.011097 & -0.213213 & 4.158832 \\
\hline 93 & 6 & 0 & 0.748870 & -0.030139 & 3.274463 \\
\hline 94 & 1 & 0 & 1.350539 & 0.892424 & 3.257698 \\
\hline 95 & 1 & 0 & 0.315990 & -0.123937 & 4.279908 \\
\hline 96 & 1 & 0 & 1.434925 & -0.879372 & 3.128220 \\
\hline 97 & 6 & 0 & 6.302443 & 0.067637 & 0.499737 \\
\hline 98 & 1 & 0 & 6.753345 & -0.842783 & 0.919424 \\
\hline 99 & 1 & 0 & 6.535691 & 0.095928 & -0.574197 \\
\hline 100 & 1 & 0 & 6.790470 & 0.930958 & 0.974628 \\
\hline 101 & 6 & 0 & 4.463056 & -5.283445 & -6.166502 \\
\hline 102 & 1 & 0 & 4.532699 & -4.354521 & -6.739540 \\
\hline 103 & 1 & 0 & 5.451071 & -5.743678 & -6.111926 \\
\hline 104 & 1 & 0 & 3.775806 & -5.974715 & -6.667138 \\
\hline 105 & 6 & 0 & 2.997245 & 0.133165 & -3.063354 \\
\hline 106 & 6 & 0 & 3.150387 & -1.184875 & -3.830764 \\
\hline
\end{tabular}




$\begin{array}{llllll}107 & 6 & 0 & 2.293230 & -1.230396 & -5.102546 \\ 108 & 6 & 0 & 2.068386 & -3.806129 & -5.574685 \\ 109 & 6 & 0 & 2.565201 & -2.413595 & -6.049118 \\ 110 & 1 & 0 & 1.937073 & 0.270759 & -2.787865 \\ 111 & 1 & 0 & 2.890236 & -2.012691 & -3.164640 \\ 112 & 1 & 0 & 1.226734 & -1.222875 & -4.834629 \\ 113 & 1 & 0 & 1.055422 & -3.711912 & -5.173390 \\ 114 & 1 & 0 & 3.256384 & 0.971876 & -3.730069 \\ 115 & 1 & 0 & 4.211941 & -1.304700 & -4.090167 \\ 116 & 1 & 0 & 2.465687 & -0.309181 & -5.678103 \\ 117 & 1 & 0 & 2.013298 & -4.472841 & -6.443427 \\ 118 & 1 & 0 & 2.059930 & -2.212932 & -7.002353 \\ 119 & 1 & 0 & 3.638557 & -2.450055 & -6.276704 \\ 120 & 6 & 0 & 2.891820 & -4.470652 & -4.480783 \\ 121 & 8 & 0 & 2.554414 & -4.492929 & -3.317638 \\ 122 & 8 & 0 & 3.835332 & 0.173581 & -1.934221\end{array}$




\section{Table S22. Thermochemistry data of IV}

- Thermochemistry -

Temperature 298.150 Kelvin. Pressure 1.00000 Atm.

Molecular mass: $\quad 838.44225 \mathrm{amu}$.

\begin{tabular}{|c|c|c|}
\hline & (Hartree/Particle) & $(\mathrm{kcal} / \mathrm{mol})$ \\
\hline Zero-point correction $=$ & 1.033801 & \\
\hline Thermal correction to Energy= & 1.100465 & \\
\hline Thermal correction to Enthalpy= & 1.101409 & \\
\hline Thermal correction to Gibbs Free Energy= & 0.929218 & \\
\hline Electronic Energy= & -3217.707734 & -2019142.088 \\
\hline Sum of electronic and zero-point Energies $=$ & -3216.673933 & -2018493.368 \\
\hline Sum of electronic and thermal Energies $=$ & -3216.607269 & -2018451.536 \\
\hline Sum of electronic and thermal Enthalpies $=$ & -3216.606325 & -2018450.943 \\
\hline \multicolumn{2}{|c|}{ Sum of electronic and thermal Free Energies $=$} & -3216.778516 \\
\hline & -2018558.995 & \\
\hline E (Thermal) & $\mathrm{CV}$ & $\mathrm{S}$ \\
\hline $\mathrm{KCal} / \mathrm{Mol}$ & Cal/Mol-Kelvin & $\mathrm{Cal} /$ Mol-Kelvin \\
\hline 690.552 & 245.672 & 362.408 \\
\hline
\end{tabular}


Transition state $\mathrm{c} 1$

Table S23. Atomic coordinates of TSc1

$\begin{array}{lcccc}\text { Center } & \text { Atomic } & \text { Atomic } & \text { Coordinates (Angstroms) } \\ \text { Number } & \text { Number } & \text { Type } & X & \text { Y } \\ Z & & & \end{array}$

\begin{tabular}{|c|c|c|c|c|c|}
\hline 1 & 13 & 0 & 0.211693 & 0.143074 & 0.354784 \\
\hline 2 & 7 & 0 & 1.417198 & 1.653494 & -0.387705 \\
\hline 3 & 6 & 0 & 0.841212 & 2.861716 & -0.358747 \\
\hline 4 & 6 & 0 & 3.033540 & 3.176193 & -0.467294 \\
\hline 5 & 6 & 0 & 1.814795 & 3.855166 & -0.428910 \\
\hline 6 & 1 & 0 & 1.660542 & 4.922054 & -0.430533 \\
\hline 7 & 7 & 0 & -1.141765 & 1.634494 & 0.022147 \\
\hline 8 & 6 & 0 & -0.599131 & 2.844334 & -0.190991 \\
\hline 9 & 6 & 0 & -2.794802 & 3.114080 & -0.037938 \\
\hline 10 & 6 & 0 & -1.599393 & 3.810789 & -0.217391 \\
\hline 11 & 1 & 0 & -1.473337 & 4.874208 & -0.343468 \\
\hline 12 & 7 & 0 & 1.520879 & -1.127835 & -0.487538 \\
\hline 13 & 6 & 0 & 1.002337 & -2.191332 & -1.117433 \\
\hline 14 & 6 & 0 & 1.978138 & -3.179599 & -1.242116 \\
\hline 15 & 6 & 0 & 3.131574 & -2.635490 & -0.667263 \\
\hline 16 & 1 & 0 & 1.859399 & -4.166551 & -1.661011 \\
\hline 17 & 7 & 0 & -0.984456 & -0.945878 & -0.976798 \\
\hline 18 & 6 & 0 & -0.405036 & -2.057310 & -1.447335 \\
\hline 19 & 6 & 0 & -2.548624 & -2.143716 & -1.997478 \\
\hline 20 & 6 & 0 & -1.345394 & -2.838170 & -2.114725 \\
\hline 21 & 1 & 0 & -1.174937 & -3.771308 & -2.627065 \\
\hline 22 & 7 & 0 & 2.848472 & -1.363334 & -0.237892 \\
\hline 23 & 7 & 0 & 2.776524 & 1.824633 & -0.431288 \\
\hline 24 & 7 & 0 & -2.506217 & 1.777196 & 0.073676 \\
\hline 25 & 7 & 0 & -2.320868 & -0.992510 & -1.277555 \\
\hline 26 & 13 & 0 & 3.939961 & 0.246723 & -0.534341 \\
\hline 27 & 13 & 0 & -3.538045 & 0.146004 & -0.238019 \\
\hline 28 & 6 & 0 & 4.466421 & -3.342203 & -0.450052 \\
\hline 29 & 6 & 0 & 4.411754 & 3.835027 & -0.468931 \\
\hline 30 & 6 & 0 & -3.878802 & -2.564566 & -2.614331 \\
\hline
\end{tabular}




\begin{tabular}{|c|c|c|c|c|c|}
\hline 31 & 6 & 0 & -4.178816 & 3.750816 & 0.044585 \\
\hline 32 & 6 & 0 & 5.503188 & -2.943383 & -1.526605 \\
\hline 33 & 1 & 0 & 5.693060 & -1.869122 & -1.549347 \\
\hline 34 & 1 & 0 & 6.448636 & -3.466004 & -1.336839 \\
\hline 35 & 1 & 0 & 5.151637 & -3.231433 & -2.524322 \\
\hline 36 & 6 & 0 & 4.998947 & -3.021842 & 0.964767 \\
\hline 37 & 1 & 0 & 5.959602 & -3.527267 & 1.121133 \\
\hline 38 & 1 & 0 & 5.138917 & -1.949180 & 1.114273 \\
\hline 39 & 1 & 0 & 4.299852 & -3.377727 & 1.730491 \\
\hline 40 & 6 & 0 & 4.249173 & -4.868677 & -0.547708 \\
\hline 41 & 1 & 0 & 3.501128 & -5.217311 & 0.173104 \\
\hline 42 & 1 & 0 & 3.930899 & -5.173909 & -1.550895 \\
\hline 43 & 1 & 0 & 5.192027 & -5.383798 & -0.332777 \\
\hline 44 & 6 & 0 & 5.294011 & 3.329092 & -1.631190 \\
\hline 45 & 1 & 0 & 5.467148 & 2.252170 & -1.609330 \\
\hline 46 & 1 & 0 & 4.829306 & 3.563316 & -2.596360 \\
\hline 47 & 1 & 0 & 6.267624 & 3.832274 & -1.595537 \\
\hline 48 & 6 & 0 & 5.097442 & 3.588467 & 0.896626 \\
\hline 49 & 1 & 0 & 4.510309 & 4.039706 & 1.705237 \\
\hline 50 & 1 & 0 & 5.201973 & 2.522272 & 1.105545 \\
\hline 51 & 1 & 0 & 6.091831 & 4.051502 & 0.902674 \\
\hline 52 & 6 & 0 & 4.247867 & 5.360464 & -0.647272 \\
\hline 53 & 1 & 0 & 3.744900 & 5.608591 & -1.588833 \\
\hline 54 & 1 & 0 & 3.683166 & 5.809947 & 0.176846 \\
\hline 55 & 1 & 0 & 5.237432 & 5.830263 & -0.662370 \\
\hline 56 & 6 & 0 & -4.356344 & -1.488474 & -3.617378 \\
\hline 57 & 1 & 0 & -3.621522 & -1.365330 & -4.421578 \\
\hline 58 & 1 & 0 & -5.304652 & -1.798590 & -4.073905 \\
\hline 59 & 1 & 0 & -4.498869 & -0.523072 & -3.128657 \\
\hline 60 & 6 & 0 & -4.939236 & -2.822727 & -1.520508 \\
\hline 61 & 1 & 0 & -5.138947 & -1.935010 & -0.920042 \\
\hline 62 & 1 & 0 & -5.883709 & -3.134511 & -1.982330 \\
\hline 63 & 1 & 0 & -4.611115 & -3.619576 & -0.843538 \\
\hline 64 & 6 & 0 & -3.689130 & -3.885557 & -3.391156 \\
\hline 65 & 1 & 0 & -2.962070 & -3.776844 & -4.203251 \\
\hline 66 & 1 & 0 & -3.359058 & -4.700396 & -2.736891 \\
\hline 67 & 1 & 0 & -4.644170 & -4.183587 & -3.837722 \\
\hline 68 & 6 & 0 & -4.824222 & 3.783527 & -1.361536 \\
\hline
\end{tabular}




\begin{tabular}{|c|c|c|c|c|c|}
\hline 69 & 1 & 0 & -5.825306 & 4.229534 & -1.305861 \\
\hline 70 & 1 & 0 & -4.221776 & 4.393481 & -2.044636 \\
\hline 71 & 1 & 0 & -4.903467 & 2.776957 & -1.775784 \\
\hline 72 & 6 & 0 & -5.081924 & 3.007414 & 1.049238 \\
\hline 73 & 1 & 0 & -6.043510 & 3.526783 & 1.135647 \\
\hline 74 & 1 & 0 & -5.288149 & 1.984902 & 0.732786 \\
\hline 75 & 1 & 0 & -4.622317 & 2.976466 & 2.043520 \\
\hline 76 & 6 & 0 & -4.033415 & 5.206729 & 0.545450 \\
\hline 77 & 1 & 0 & -3.532159 & 5.251354 & 1.518734 \\
\hline 78 & 1 & 0 & -3.472675 & 5.829887 & -0.158919 \\
\hline 79 & 1 & 0 & -5.027972 & 5.653812 & 0.653770 \\
\hline 80 & 8 & 0 & 5.203197 & 0.324745 & 0.643170 \\
\hline 81 & 8 & 0 & 4.564185 & 0.142862 & -2.148579 \\
\hline 82 & 8 & 0 & 0.796322 & 0.451687 & 2.024646 \\
\hline 83 & 8 & 0 & -3.916012 & -0.641231 & 1.265345 \\
\hline 84 & 8 & 0 & -4.885704 & 0.582069 & -1.234116 \\
\hline 85 & 6 & 0 & -6.280744 & 0.450968 & -1.240112 \\
\hline 86 & 1 & 0 & -6.757725 & 1.438743 & -1.317700 \\
\hline 87 & 1 & 0 & -6.608549 & -0.142813 & -2.105535 \\
\hline 88 & 1 & 0 & -6.671189 & -0.036303 & -0.335065 \\
\hline 89 & 6 & 0 & -5.131180 & -0.828589 & 1.939895 \\
\hline 90 & 1 & 0 & -5.686077 & 0.110738 & 2.092009 \\
\hline 91 & 1 & 0 & -5.798738 & -1.527199 & 1.409654 \\
\hline 92 & 1 & 0 & -4.941493 & -1.256618 & 2.934095 \\
\hline 93 & 6 & 0 & 1.695410 & 1.362615 & 2.593944 \\
\hline 94 & 1 & 0 & 1.548506 & 1.376709 & 3.685259 \\
\hline 95 & 1 & 0 & 2.744124 & 1.085162 & 2.410962 \\
\hline 96 & 1 & 0 & 1.550269 & 2.392807 & 2.234818 \\
\hline 97 & 6 & 0 & 6.602867 & 0.302000 & 0.486954 \\
\hline 98 & 1 & 0 & 7.027053 & -0.588895 & 0.972039 \\
\hline 99 & 1 & 0 & 6.911564 & 0.292018 & -0.567804 \\
\hline 100 & 1 & 0 & 7.053148 & 1.185328 & 0.962023 \\
\hline 101 & 6 & 0 & 3.915574 & 0.188304 & -3.392478 \\
\hline 102 & 1 & 0 & 3.171699 & 0.998954 & -3.450203 \\
\hline 103 & 1 & 0 & 4.653771 & 0.358255 & -4.188685 \\
\hline 104 & 1 & 0 & 3.396679 & -0.757076 & -3.617103 \\
\hline 105 & 6 & 0 & -0.338913 & -3.646279 & 3.096630 \\
\hline 106 & 6 & 0 & -1.533481 & -3.944637 & 4.003817 \\
\hline
\end{tabular}




$\begin{array}{lllrrr}107 & 6 & 0 & -2.633827 & -2.855767 & 3.998704 \\ 108 & 6 & 0 & -1.435785 & -0.523721 & 3.713479 \\ 109 & 6 & 0 & -2.226824 & -1.499381 & 4.636108 \\ 110 & 1 & 0 & -0.586341 & -3.730719 & 2.034408 \\ 111 & 1 & 0 & -1.172691 & -4.075462 & 5.032544 \\ 112 & 1 & 0 & -2.998935 & -2.687701 & 2.976778 \\ 113 & 1 & 0 & -2.103157 & 0.114947 & 3.133280 \\ 114 & 1 & 0 & 0.516450 & -4.288601 & 3.315539 \\ 115 & 1 & 0 & -1.966269 & -4.906144 & 3.695235 \\ 116 & 1 & 0 & -3.483289 & -3.253491 & 4.567189 \\ 117 & 1 & 0 & -0.768134 & 0.106499 & 4.308141 \\ 118 & 1 & 0 & -3.134650 & -0.976576 & 4.957754 \\ 119 & 1 & 0 & -1.642734 & -1.699028 & 5.542012 \\ 120 & 6 & 0 & -0.629188 & -1.342648 & 2.751281 \\ 121 & 8 & 0 & -0.774782 & -1.353418 & 1.526781 \\ 122 & 8 & 0 & 0.100499 & -2.290814 & 3.374032\end{array}$


Table S24. Thermochemistry data of TSc1

- Thermochemistry -

Temperature 298.150 Kelvin. Pressure 1.00000 Atm.

Molecular mass: $\quad 838.44225 \mathrm{amu}$.

\begin{tabular}{|c|c|c|}
\hline & (Hartree/Particle) & (kcal/mol) \\
\hline Zero-point correction $=$ & 1.033372 & \\
\hline Thermal correction to Energy= & 1.098457 & \\
\hline Thermal correction to Enthalpy= & 1.099401 & \\
\hline Thermal correction to Gibbs Free Energy= & 0.934729 & \\
\hline Electronic Energy= & -3217.670588 & -2019118.778 \\
\hline Sum of electronic and zero-point Energies $=$ & -3216.637216 & -2018470.327 \\
\hline Sum of electronic and thermal Energies $=$ & -3216.572131 & -2018429.486 \\
\hline Sum of electronic and thermal Enthalpies $=$ & -3216.571187 & -2018428.894 \\
\hline \multicolumn{2}{|l|}{ Sum of electronic and thermal Free Energies $=$} & -3216.735859 \\
\hline & -2018532.227 & \\
\hline E (Thermal) & $\mathrm{CV}$ & $\mathrm{S}$ \\
\hline $\mathrm{KCal} / \mathrm{Mol}$ & Cal/Mol-Kelvin & Cal/Mol-Kelvin \\
\hline 689.292 & 243.644 & 346.582 \\
\hline
\end{tabular}


Intermediate cI

Table S25. Atomic coordinates of $\mathrm{cI}$

$\begin{array}{lcccc}\text { Center } & \text { Atomic } & \text { Atomic } & \text { Coordinates (Angstroms) } \\ \text { Number } & \text { Number } & \text { Type } & X & \text { Y } \\ Z & & & \end{array}$

\begin{tabular}{|c|c|c|c|c|c|}
\hline 1 & 13 & 0 & -0.247837 & -0.080717 & -0.211575 \\
\hline 2 & 7 & 0 & 1.136998 & 1.356624 & -0.195052 \\
\hline 3 & 6 & 0 & 0.630662 & 2.593302 & -0.051440 \\
\hline 4 & 6 & 0 & 2.782558 & 2.679062 & 0.474541 \\
\hline 5 & 6 & 0 & 1.629074 & 3.463745 & 0.364228 \\
\hline 6 & 1 & 0 & 1.530280 & 4.514515 & 0.583153 \\
\hline 7 & 7 & 0 & -1.410624 & 1.515305 & -0.501019 \\
\hline 8 & 6 & 0 & -0.793365 & 2.692586 & -0.307767 \\
\hline 9 & 6 & 0 & -2.934751 & 3.108818 & -0.701490 \\
\hline 10 & 6 & 0 & -1.711500 & 3.727279 & -0.425268 \\
\hline 11 & 1 & 0 & -1.519405 & 4.783899 & -0.334118 \\
\hline 12 & 7 & 0 & 1.071728 & -1.342096 & -1.050184 \\
\hline 13 & 6 & 0 & 0.504458 & -2.420992 & -1.614237 \\
\hline 14 & 6 & 0 & 1.458382 & -3.416079 & -1.789075 \\
\hline 15 & 6 & 0 & 2.649088 & -2.882086 & -1.286411 \\
\hline 16 & 1 & 0 & 1.306305 & -4.401088 & -2.199423 \\
\hline 17 & 7 & 0 & -1.485386 & -1.196923 & -1.320635 \\
\hline 18 & 6 & 0 & -0.925526 & -2.307368 & -1.830468 \\
\hline 19 & 6 & 0 & -3.093944 & -2.376200 & -2.284752 \\
\hline 20 & 6 & 0 & -1.897327 & -3.081377 & -2.448383 \\
\hline 21 & 1 & 0 & -1.758698 & -4.025503 & -2.949278 \\
\hline 22 & 7 & 0 & 2.401099 & -1.609297 & -0.839443 \\
\hline 23 & 7 & 0 & 2.471285 & 1.394520 & 0.119138 \\
\hline 24 & 7 & 0 & -2.738257 & 1.753819 & -0.749388 \\
\hline 25 & 7 & 0 & -2.828272 & -1.220553 & -1.599016 \\
\hline 26 & 13 & 0 & 3.583812 & -0.079287 & -0.522765 \\
\hline 27 & 13 & 0 & -3.934998 & 0.200413 & -0.821813 \\
\hline 28 & 6 & 0 & 3.984111 & -3.616409 & -1.189809 \\
\hline 29 & 6 & 0 & 4.142736 & 3.154127 & 0.977011 \\
\hline 30 & 6 & 0 & -4.460056 & -2.828012 & -2.792591 \\
\hline
\end{tabular}




\begin{tabular}{|c|c|c|c|c|c|}
\hline 31 & 6 & 0 & -4.261649 & 3.826065 & -0.927643 \\
\hline 32 & 6 & 0 & 5.071269 & -2.930121 & -2.047777 \\
\hline 33 & 1 & 0 & 5.254543 & -1.894341 & -1.759168 \\
\hline 34 & 1 & 0 & 6.013541 & -3.483058 & -1.954922 \\
\hline 35 & 1 & 0 & 4.783690 & -2.927574 & -3.105578 \\
\hline 36 & 6 & 0 & 4.420839 & -3.701997 & 0.291425 \\
\hline 37 & 1 & 0 & 5.370713 & -4.245151 & 0.366001 \\
\hline 38 & 1 & 0 & 4.547129 & -2.709356 & 0.727471 \\
\hline 39 & 1 & 0 & 3.674218 & -4.247249 & 0.880833 \\
\hline 40 & 6 & 0 & 3.813793 & -5.056215 & -1.720552 \\
\hline 41 & 1 & 0 & 3.071625 & -5.617936 & -1.142617 \\
\hline 42 & 1 & 0 & 3.516150 & -5.069731 & -2.775131 \\
\hline 43 & 1 & 0 & 4.769271 & -5.585328 & -1.638367 \\
\hline 44 & 6 & 0 & 5.228746 & 3.025203 & -0.114918 \\
\hline 45 & 1 & 0 & 5.366139 & 2.000633 & -0.462511 \\
\hline 46 & 1 & 0 & 4.974094 & 3.634736 & -0.989457 \\
\hline 47 & 1 & 0 & 6.187914 & 3.381441 & 0.279147 \\
\hline 48 & 6 & 0 & 4.530568 & 2.350691 & 2.240495 \\
\hline 49 & 1 & 0 & 3.778217 & 2.483599 & 3.026220 \\
\hline 50 & 1 & 0 & 4.611241 & 1.283880 & 2.024638 \\
\hline 51 & 1 & 0 & 5.492906 & 2.709514 & 2.625817 \\
\hline 52 & 6 & 0 & 4.050553 & 4.645223 & 1.367031 \\
\hline 53 & 1 & 0 & 3.792140 & 5.277249 & 0.509650 \\
\hline 54 & 1 & 0 & 3.311496 & 4.813486 & 2.158138 \\
\hline 55 & 1 & 0 & 5.022866 & 4.979989 & 1.744334 \\
\hline 56 & 6 & 0 & -4.929198 & -1.909147 & -3.945429 \\
\hline 57 & 1 & 0 & -4.217521 & -1.947631 & -4.778759 \\
\hline 58 & 1 & 0 & -5.901650 & -2.252969 & -4.319184 \\
\hline 59 & 1 & 0 & -5.024544 & -0.874217 & -3.612615 \\
\hline 60 & 6 & 0 & -5.489346 & -2.854101 & -1.641056 \\
\hline 61 & 1 & 0 & -5.643316 & -1.871727 & -1.194482 \\
\hline 62 & 1 & 0 & -6.457259 & -3.204177 & -2.018993 \\
\hline 63 & 1 & 0 & -5.167294 & -3.535968 & -0.846267 \\
\hline 64 & 6 & 0 & -4.348458 & -4.265419 & -3.346766 \\
\hline 65 & 1 & 0 & -3.668928 & -4.320900 & -4.204526 \\
\hline 66 & 1 & 0 & -4.003435 & -4.972375 & -2.583804 \\
\hline 67 & 1 & 0 & -5.335182 & -4.596402 & -3.688053 \\
\hline 68 & 6 & 0 & -4.729286 & 3.627167 & -2.388712 \\
\hline
\end{tabular}




\begin{tabular}{|c|c|c|c|c|c|}
\hline 69 & 1 & 0 & -5.674372 & 4.160140 & -2.550531 \\
\hline 70 & 1 & 0 & -3.989401 & 4.037584 & -3.086048 \\
\hline 71 & 1 & 0 & -4.877175 & 2.570186 & -2.617918 \\
\hline 72 & 6 & 0 & -5.327781 & 3.327372 & 0.073854 \\
\hline 73 & 1 & 0 & -6.270149 & 3.862928 & -0.090772 \\
\hline 74 & 1 & 0 & -5.531109 & 2.260429 & -0.026637 \\
\hline 75 & 1 & 0 & -5.007459 & 3.513821 & 1.105151 \\
\hline 76 & 6 & 0 & -4.072761 & 5.339677 & -0.688340 \\
\hline 77 & 1 & 0 & -3.733502 & 5.553304 & 0.331670 \\
\hline 78 & 1 & 0 & -3.354823 & 5.776309 & -1.391395 \\
\hline 79 & 1 & 0 & -5.030503 & 5.850431 & -0.835172 \\
\hline 80 & 8 & 0 & 4.773973 & -0.491121 & 0.659799 \\
\hline 81 & 8 & 0 & 4.279043 & 0.372794 & -2.042148 \\
\hline 82 & 8 & 0 & -0.580550 & 1.079158 & 2.642971 \\
\hline 83 & 8 & 0 & -4.465448 & -0.230049 & 0.752317 \\
\hline 84 & 8 & 0 & -5.218138 & 0.516511 & -1.937252 \\
\hline 85 & 6 & 0 & -6.615810 & 0.547660 & -1.785249 \\
\hline 86 & 1 & 0 & -7.005022 & 1.545520 & -2.034117 \\
\hline 87 & 1 & 0 & -7.088668 & -0.172624 & -2.468162 \\
\hline 88 & 1 & 0 & -6.934729 & 0.303915 & -0.761989 \\
\hline 89 & 6 & 0 & -4.201253 & 0.180157 & 2.068603 \\
\hline 90 & 1 & 0 & -3.230902 & 0.684320 & 2.169582 \\
\hline 91 & 1 & 0 & -4.984746 & 0.865934 & 2.427980 \\
\hline 92 & 1 & 0 & -4.196306 & -0.693363 & 2.735530 \\
\hline 93 & 6 & 0 & -0.304260 & 1.849637 & 3.802254 \\
\hline 94 & 1 & 0 & -0.943995 & 1.562252 & 4.648566 \\
\hline 95 & 1 & 0 & 0.747103 & 1.770387 & 4.096999 \\
\hline 96 & 1 & 0 & -0.528742 & 2.886449 & 3.536964 \\
\hline 97 & 6 & 0 & 6.181243 & -0.506483 & 0.612609 \\
\hline 98 & 1 & 0 & 6.558211 & -1.510327 & 0.854583 \\
\hline 99 & 1 & 0 & 6.574036 & -0.226412 & -0.374620 \\
\hline 100 & 1 & 0 & 6.594419 & 0.192972 & 1.353063 \\
\hline 101 & 6 & 0 & 3.687597 & 0.644073 & -3.285858 \\
\hline 102 & 1 & 0 & 3.123167 & 1.590095 & -3.277126 \\
\hline 103 & 1 & 0 & 4.467820 & 0.731607 & -4.054311 \\
\hline 104 & 1 & 0 & 2.996258 & -0.153079 & -3.604035 \\
\hline 105 & 6 & 0 & 1.955690 & -1.464840 & 2.914443 \\
\hline 106 & 6 & 0 & 2.191238 & -1.863760 & 4.370762 \\
\hline
\end{tabular}




$\begin{array}{lllrll}107 & 6 & 0 & 0.930401 & -2.303646 & 5.123830 \\ 108 & 6 & 0 & -0.883858 & -1.140836 & 3.698484 \\ 109 & 6 & 0 & -0.227157 & -1.292742 & 5.082824 \\ 110 & 1 & 0 & 1.423345 & -2.250129 & 2.361920 \\ 111 & 1 & 0 & 2.652892 & -1.007548 & 4.881448 \\ 112 & 1 & 0 & 0.575430 & -3.259735 & 4.710162 \\ 113 & 1 & 0 & -1.024427 & -2.136291 & 3.262988 \\ 114 & 1 & 0 & 2.916309 & -1.300173 & 2.417192 \\ 115 & 1 & 0 & 2.931588 & -2.676393 & 4.391536 \\ 116 & 1 & 0 & 1.194899 & -2.504269 & 6.170938 \\ 117 & 1 & 0 & -1.888323 & -0.716771 & 3.800810 \\ 118 & 1 & 0 & -1.003834 & -1.634606 & 5.780173 \\ 119 & 1 & 0 & 0.120019 & -0.325474 & 5.464590 \\ 120 & 6 & 0 & -0.159614 & -0.282521 & 2.624953 \\ 121 & 8 & 0 & -0.495530 & -0.761841 & 1.387396 \\ 122 & 8 & 0 & 1.250525 & -0.222322 & 2.807107\end{array}$


Table S26. Thermochemical data of $\mathrm{cI}$

- Thermochemistry -

Temperature 298.150 Kelvin. Pressure 1.00000 Atm.

Molecular mass: $\quad 838.44225 \mathrm{amu}$.

\begin{tabular}{|c|c|c|}
\hline & (Hartree/Particle) & $(\mathrm{kcal} / \mathrm{mol})$ \\
\hline Zero-point correction= & 1.035794 & \\
\hline Thermal correction to Energy= & 1.100666 & \\
\hline Thermal correction to Enthalpy= & 1.10161 & \\
\hline Thermal correction to Gibbs Free Energy= & 0.93681 & \\
\hline Electronic Energy= & -3217.707244 & -2019141.780 \\
\hline Sum of electronic and zero-point Energies $=$ & -3216.671450 & -2018491.809 \\
\hline Sum of electronic and thermal Energies $=$ & -3216.606578 & -2018451.102 \\
\hline Sum of electronic and thermal Enthalpies $=$ & -3216.605634 & -2018450.509 \\
\hline \multicolumn{2}{|c|}{ Sum of electronic and thermal Free Energies $=$} & -3216.770434 \\
\hline & -2018553.923 & \\
\hline E (Thermal) & $\mathrm{CV}$ & $\mathrm{S}$ \\
\hline $\mathrm{KCal} / \mathrm{Mol}$ & Cal/Mol-Kelvin & Cal/Mol-Kelvin \\
\hline 690.678 & 243.442 & 346.851 \\
\hline
\end{tabular}


Transition state $\mathrm{c} 2$

Table S27. Atomic coordinate of TSc2

\begin{tabular}{|c|c|c|c|c|c|}
\hline \multirow{3}{*}{$\begin{array}{l}\text { Center } \\
\text { Number } \\
\text { Z }\end{array}$} & \multirow{3}{*}{$\begin{array}{l}\text { Atomic } \\
\text { Number }\end{array}$} & \multirow{3}{*}{$\begin{array}{l}\text { Atomic } \\
\text { Type }\end{array}$} & \multicolumn{3}{|c|}{ Coordinates (Angstroms) } \\
\hline & & & $\mathrm{X}$ & $\mathrm{Y}$ & \\
\hline & & & & & \\
\hline 1 & 7 & 0 & 0.733915 & 1.269850 & -0.190177 \\
\hline 2 & 6 & 0 & 0.215699 & 2.282866 & -0.899490 \\
\hline 3 & 6 & 0 & 2.337914 & 2.774601 & -0.464932 \\
\hline 4 & 6 & 0 & 1.187953 & 3.265976 & -1.088027 \\
\hline 5 & 1 & 0 & 1.065490 & 4.218816 & -1.578575 \\
\hline 6 & 7 & 0 & -1.789043 & 1.077861 & -0.655466 \\
\hline 7 & 6 & 0 & -1.190511 & 2.131546 & -1.228518 \\
\hline 8 & 6 & 0 & -3.326588 & 2.178634 & -1.818678 \\
\hline 9 & 6 & 0 & -2.113848 & 2.849703 & -1.983151 \\
\hline 10 & 1 & 0 & -1.929341 & 3.731081 & -2.575887 \\
\hline 11 & 7 & 0 & 0.655532 & -1.506067 & -0.016817 \\
\hline 12 & 6 & 0 & 0.096444 & -2.717378 & 0.089452 \\
\hline 13 & 6 & 0 & 1.081704 & -3.695048 & 0.195196 \\
\hline 14 & 6 & 0 & 2.290330 & -2.999309 & 0.190561 \\
\hline 15 & 1 & 0 & 0.939044 & -4.758906 & 0.294552 \\
\hline 16 & 7 & 0 & -1.906228 & -1.505127 & 0.381901 \\
\hline 17 & 6 & 0 & -1.347971 & -2.712084 & 0.198559 \\
\hline 18 & 6 & 0 & -3.545741 & -2.999766 & 0.270280 \\
\hline 19 & 6 & 0 & -2.339174 & -3.687380 & 0.142747 \\
\hline 20 & 1 & 0 & -2.199167 & -4.750346 & 0.028890 \\
\hline 21 & 7 & 0 & 2.015409 & -1.656734 & 0.072828 \\
\hline 22 & 7 & 0 & 2.057111 & 1.533743 & 0.055898 \\
\hline 23 & 7 & 0 & -3.118753 & 1.098934 & -0.993679 \\
\hline 24 & 7 & 0 & -3.272434 & -1.660228 & 0.384230 \\
\hline 25 & 13 & 0 & 3.167155 & -0.087585 & -0.100525 \\
\hline 26 & 13 & 0 & -4.301901 & -0.032080 & 0.093009 \\
\hline 27 & 6 & 0 & 3.672916 & -3.625034 & 0.357494 \\
\hline 28 & 6 & 0 & 3.664727 & 3.508936 & -0.291900 \\
\hline 29 & 6 & 0 & -4.931505 & -3.637375 & 0.310494 \\
\hline
\end{tabular}




\begin{tabular}{|c|c|c|c|c|c|}
\hline 30 & 6 & 0 & -4.653591 & 2.578402 & -2.456062 \\
\hline 31 & 6 & 0 & 4.625412 & -3.229925 & -0.792949 \\
\hline 32 & 1 & 0 & 4.766639 & -2.152512 & -0.891446 \\
\hline 33 & 1 & 0 & 5.607790 & -3.688782 & -0.628949 \\
\hline 34 & 1 & 0 & 4.239311 & -3.594496 & -1.752309 \\
\hline 35 & 6 & 0 & 4.256725 & -3.217671 & 1.731596 \\
\hline 36 & 1 & 0 & 5.251061 & -3.663075 & 1.861497 \\
\hline 37 & 1 & 0 & 4.339312 & -2.133460 & 1.826774 \\
\hline 38 & 1 & 0 & 3.613942 & -3.582946 & 2.541077 \\
\hline 39 & 6 & 0 & 3.543454 & -5.164017 & 0.336871 \\
\hline 40 & 1 & 0 & 2.927180 & -5.532290 & 1.164308 \\
\hline 41 & 1 & 0 & 3.111595 & -5.523487 & -0.604081 \\
\hline 42 & 1 & 0 & 4.537830 & -5.611732 & 0.441765 \\
\hline 43 & 6 & 0 & 4.717608 & 3.031519 & -1.319785 \\
\hline 44 & 1 & 0 & 4.917737 & 1.961351 & -1.248808 \\
\hline 45 & 1 & 0 & 4.374085 & 3.230016 & -2.341734 \\
\hline 46 & 1 & 0 & 5.655178 & 3.579350 & -1.165371 \\
\hline 47 & 6 & 0 & 4.186223 & 3.311382 & 1.149208 \\
\hline 48 & 1 & 0 & 3.476036 & 3.723748 & 1.876374 \\
\hline 49 & 1 & 0 & 4.337649 & 2.255694 & 1.384464 \\
\hline 50 & 1 & 0 & 5.138931 & 3.840045 & 1.272840 \\
\hline 51 & 6 & 0 & 3.436347 & 5.020074 & -0.517357 \\
\hline 52 & 1 & 0 & 3.126522 & 5.238388 & -1.545516 \\
\hline 53 & 1 & 0 & 2.677775 & 5.421410 & 0.164139 \\
\hline 54 & 1 & 0 & 4.372698 & 5.559574 & -0.336879 \\
\hline 55 & 6 & 0 & -5.591292 & -3.571016 & -1.087736 \\
\hline 56 & 1 & 0 & -4.983212 & -4.110972 & -1.822938 \\
\hline 57 & 1 & 0 & -6.580435 & -4.045198 & -1.056402 \\
\hline 58 & 1 & 0 & -5.703306 & -2.537096 & -1.419544 \\
\hline 59 & 6 & 0 & -5.820552 & -2.958486 & 1.373619 \\
\hline 60 & 1 & 0 & -6.006349 & -1.907889 & 1.146896 \\
\hline 61 & 1 & 0 & -6.793091 & -3.462520 & 1.418868 \\
\hline 62 & 1 & 0 & -5.360327 & -3.020285 & 2.366170 \\
\hline 63 & 6 & 0 & -4.790608 & -5.125016 & 0.705620 \\
\hline 64 & 1 & 0 & -4.236383 & -5.697108 & -0.046085 \\
\hline 65 & 1 & 0 & -4.284941 & -5.242455 & 1.670561 \\
\hline 66 & 1 & 0 & -5.786958 & -5.573800 & 0.786559 \\
\hline 67 & 6 & 0 & -5.185602 & 1.439749 & -3.356833 \\
\hline
\end{tabular}




\begin{tabular}{|c|c|c|c|c|c|}
\hline 68 & 1 & 0 & -6.121550 & 1.754612 & -3.834868 \\
\hline 69 & 1 & 0 & -4.464025 & 1.211905 & -4.150302 \\
\hline 70 & 1 & 0 & -5.374577 & 0.531414 & -2.782234 \\
\hline 71 & 6 & 0 & -5.681140 & 2.956624 & -1.364967 \\
\hline 72 & 1 & 0 & -6.629961 & 3.249081 & -1.830837 \\
\hline 73 & 1 & 0 & -5.884275 & 2.130298 & -0.683360 \\
\hline 74 & 1 & 0 & -5.320284 & 3.803303 & -0.769952 \\
\hline 75 & 6 & 0 & -4.440537 & 3.823328 & -3.344325 \\
\hline 76 & 1 & 0 & -4.080362 & 4.682378 & -2.767002 \\
\hline 77 & 1 & 0 & -3.729244 & 3.627100 & -4.154351 \\
\hline 78 & 1 & 0 & -5.394440 & 4.107430 & -3.801955 \\
\hline 79 & 8 & 0 & 4.411005 & -0.027704 & 1.101190 \\
\hline 80 & 8 & 0 & -4.630234 & 0.779823 & 1.583671 \\
\hline 81 & 8 & 0 & -5.708749 & -0.403152 & -0.844189 \\
\hline 82 & 6 & 0 & -7.082384 & -0.223579 & -0.609395 \\
\hline 83 & 1 & 0 & -7.603271 & -1.192002 & -0.624665 \\
\hline 84 & 1 & 0 & -7.286160 & 0.256269 & 0.358946 \\
\hline 85 & 1 & 0 & -7.525306 & 0.402728 & -1.397039 \\
\hline 86 & 6 & 0 & -4.617887 & 0.343938 & 2.917677 \\
\hline 87 & 1 & 0 & -4.356160 & 1.182274 & 3.580132 \\
\hline 88 & 1 & 0 & -5.609530 & -0.021078 & 3.227997 \\
\hline 89 & 1 & 0 & -3.887257 & -0.458516 & 3.093222 \\
\hline 90 & 6 & 0 & 5.811991 & -0.036823 & 0.953830 \\
\hline 91 & 1 & 0 & 6.127181 & -0.070383 & -0.098321 \\
\hline 92 & 1 & 0 & 6.247119 & 0.865093 & 1.408241 \\
\hline 93 & 1 & 0 & 6.245286 & -0.908799 & 1.464730 \\
\hline 94 & 6 & 0 & 3.209128 & -0.240582 & -2.950867 \\
\hline 95 & 1 & 0 & 3.962287 & -0.477462 & -3.715384 \\
\hline 96 & 1 & 0 & 2.464251 & -1.052172 & -2.954475 \\
\hline 97 & 8 & 0 & 3.833290 & -0.089681 & -1.702610 \\
\hline 98 & 13 & 0 & -0.594659 & 0.023366 & 0.688117 \\
\hline 99 & 8 & 0 & -1.060078 & -0.421750 & 4.066124 \\
\hline 100 & 6 & 0 & -1.420564 & 0.038645 & 5.366403 \\
\hline 101 & 1 & 0 & -0.541458 & 0.182653 & 6.002256 \\
\hline 102 & 1 & 0 & -2.005865 & 0.961009 & 5.319354 \\
\hline 103 & 1 & 0 & -2.038391 & -0.756402 & 5.787494 \\
\hline 104 & 6 & 0 & -1.845651 & 2.484315 & 2.031060 \\
\hline 105 & 6 & 0 & -1.698024 & 3.261023 & 3.335624 \\
\hline
\end{tabular}




$\begin{array}{lrrrrr}106 & 6 & 0 & -0.250520 & 3.582495 & 3.744677 \\ 107 & 6 & 0 & 0.839558 & 1.202496 & 3.826944 \\ 108 & 6 & 0 & 0.465417 & 2.499251 & 4.564008 \\ 109 & 1 & 0 & -1.388067 & 3.088008 & 1.234881 \\ 110 & 1 & 0 & -2.216458 & 2.737184 & 4.150556 \\ 111 & 1 & 0 & 0.337690 & 3.813570 & 2.844776 \\ 112 & 1 & 0 & 1.470566 & 1.454401 & 2.970625 \\ 113 & 1 & 0 & -2.908110 & 2.358131 & 1.798164 \\ 114 & 1 & 0 & -2.247362 & 4.201639 & 3.191562 \\ 115 & 1 & 0 & -0.249695 & 4.497705 & 4.351366 \\ 116 & 1 & 0 & 1.470398 & 0.589124 & 4.489370 \\ 117 & 1 & 0 & 1.418415 & 2.917299 & 4.915062 \\ 118 & 1 & 0 & -0.113040 & 2.299097 & 5.471840 \\ 119 & 6 & 0 & -0.176910 & 0.210147 & 3.269136 \\ 120 & 8 & 0 & 0.229562 & -0.538296 & 2.304289 \\ 121 & 8 & 0 & -1.228620 & 1.199696 & 2.053629 \\ 122 & 1 & 0 & 2.696703 & 0.682901 & -3.264787 \\ ------------ & & \end{array}$


Table S28. Thermochemistry data of TSc2

- Thermochemistry -

Temperature 298.150 Kelvin. Pressure 1.00000 Atm.

Molecular mass: $\quad 838.44225 \mathrm{amu}$.

\begin{tabular}{|c|c|c|}
\hline & (Hartree/Particle) & $(\mathrm{kcal} / \mathrm{mol})$ \\
\hline Zero-point correction $=$ & 1.034671 & \\
\hline Thermal correction to Energy= & 1.098952 & \\
\hline Thermal correction to Enthalpy= & 1.099897 & \\
\hline Thermal correction to Gibbs Free Energy= & 0.938405 & \\
\hline Electronic Energy= & -3217.676282 & -2019122.351 \\
\hline Sum of electronic and zero-point Energies $=$ & -3216.641611 & -2018473.085 \\
\hline Sum of electronic and thermal Energies $=$ & -3216.577330 & -2018432.748 \\
\hline Sum of electronic and thermal Enthalpies $=$ & -3216.576385 & -2018432.155 \\
\hline \multicolumn{2}{|l|}{ Sum of electronic and thermal Free Energies $=$} & -3216.737877 \\
\hline & -2018533.493 & \\
\hline E (Thermal) & $\mathrm{CV}$ & $\mathrm{S}$ \\
\hline KCal/Mol & Cal/Mol-Kelvin & Cal/Mol-Kelvin \\
\hline 689.603 & 242.776 & 339.887 \\
\hline
\end{tabular}


Intermediate cII

Table S29. Atomic coordinates of cII

Standard orientation:

$\begin{array}{lcccc}\text { Center } & \text { Atomic } & \text { Atomic } & \text { Coordinates (Angstroms) } \\ \text { Number } & \text { Number } & \text { Type } & X & \text { Y } \\ Z & & & & \end{array}$

\begin{tabular}{|c|c|c|c|c|c|}
\hline 1 & 7 & 0 & -1.298244 & -1.245183 & -0.481351 \\
\hline 2 & 6 & 0 & -0.789425 & -2.382524 & -0.977265 \\
\hline 3 & 6 & 0 & -2.958046 & -2.710344 & -0.616938 \\
\hline 4 & 6 & 0 & -1.799219 & -3.342476 & -1.071172 \\
\hline 5 & 1 & 0 & -1.694551 & -4.368485 & -1.387170 \\
\hline 6 & 7 & 0 & 1.261307 & -1.246053 & -0.795483 \\
\hline 7 & 6 & 0 & 0.643856 & -2.359799 & -1.211117 \\
\hline 8 & 6 & 0 & 2.817508 & -2.641206 & -1.542808 \\
\hline 9 & 6 & 0 & 1.579773 & -3.263612 & -1.708139 \\
\hline 10 & 1 & 0 & 1.388344 & -4.236666 & -2.130755 \\
\hline 11 & 7 & 0 & -1.057441 & 1.489749 & -0.790021 \\
\hline 12 & 6 & 0 & -0.433940 & 2.668932 & -0.896050 \\
\hline 13 & 6 & 0 & -1.360053 & 3.687736 & -1.096808 \\
\hline 14 & 6 & 0 & -2.606478 & 3.063155 & -1.062648 \\
\hline 15 & 1 & 0 & -1.157336 & 4.739407 & -1.218439 \\
\hline 16 & 7 & 0 & 1.453221 & 1.460927 & -0.169810 \\
\hline 17 & 6 & 0 & 0.993022 & 2.624669 & -0.654249 \\
\hline 18 & 6 & 0 & 3.192014 & 2.812974 & -0.445570 \\
\hline 19 & 6 & 0 & 2.047496 & 3.516175 & -0.823140 \\
\hline 20 & 1 & 0 & 1.988332 & 4.535483 & -1.169556 \\
\hline 21 & 7 & 0 & -2.409227 & 1.715487 & -0.863051 \\
\hline 22 & 7 & 0 & -2.646036 & -1.410993 & -0.288618 \\
\hline 23 & 7 & 0 & 2.611748 & -1.407467 & -0.969980 \\
\hline 24 & 7 & 0 & 2.821989 & 1.543980 & -0.079627 \\
\hline 25 & 13 & 0 & -3.655581 & 0.209254 & -0.753840 \\
\hline 26 & 13 & 0 & 3.764864 & -0.165641 & 0.030504 \\
\hline 27 & 6 & 0 & -3.951903 & 3.782765 & -1.148477 \\
\hline 28 & 6 & 0 & -4.324706 & -3.356251 & -0.402406 \\
\hline 29 & 6 & 0 & 4.608744 & 3.378255 & -0.408206 \\
\hline
\end{tabular}




\begin{tabular}{|c|c|c|c|c|c|}
\hline 30 & 6 & 0 & 4.167003 & -3.232824 & -1.937538 \\
\hline 31 & 6 & 0 & -4.862748 & 3.192945 & -2.247400 \\
\hline 32 & 1 & 0 & -5.093391 & 2.136566 & -2.101847 \\
\hline 33 & 1 & 0 & -5.807442 & 3.749176 & -2.274634 \\
\hline 34 & 1 & 0 & -4.387945 & 3.288649 & -3.231140 \\
\hline 35 & 6 & 0 & -4.644439 & 3.729406 & 0.234462 \\
\hline 36 & 1 & 0 & -5.618157 & 4.232629 & 0.184878 \\
\hline 37 & 1 & 0 & -4.793241 & 2.700885 & 0.567663 \\
\hline 38 & 1 & 0 & -4.034553 & 4.245491 & 0.985369 \\
\hline 39 & 6 & 0 & -3.715596 & 5.268314 & -1.500520 \\
\hline 40 & 1 & 0 & -3.127792 & 5.782378 & -0.732301 \\
\hline 41 & 1 & 0 & -3.203437 & 5.381644 & -2.462682 \\
\hline 42 & 1 & 0 & -4.681970 & 5.779198 & -1.572529 \\
\hline 43 & 6 & 0 & -5.300321 & -3.033240 & -1.558533 \\
\hline 44 & 1 & 0 & -5.449847 & -1.960473 & -1.692260 \\
\hline 45 & 1 & 0 & -4.915527 & -3.429500 & -2.505733 \\
\hline 46 & 1 & 0 & -6.269914 & -3.507711 & -1.363835 \\
\hline 47 & 6 & 0 & -4.911763 & -2.889697 & 0.948380 \\
\hline 48 & 1 & 0 & -4.264599 & -3.200044 & 1.777859 \\
\hline 49 & 1 & 0 & -5.017300 & -1.803847 & 0.992672 \\
\hline 50 & 1 & 0 & -5.897381 & -3.345252 & 1.101542 \\
\hline 51 & 6 & 0 & -4.152966 & -4.890650 & -0.345176 \\
\hline 52 & 1 & 0 & -3.804919 & -5.298984 & -1.300557 \\
\hline 53 & 1 & 0 & -3.445936 & -5.190437 & 0.436342 \\
\hline 54 & 1 & 0 & -5.120168 & -5.355725 & -0.124006 \\
\hline 55 & 6 & 0 & 5.373437 & 2.996506 & -1.698377 \\
\hline 56 & 1 & 0 & 4.861846 & 3.401792 & -2.579268 \\
\hline 57 & 1 & 0 & 6.384578 & 3.421943 & -1.671838 \\
\hline 58 & 1 & 0 & 5.446404 & 1.912543 & -1.804735 \\
\hline 59 & 6 & 0 & 5.361101 & 2.890872 & 0.848014 \\
\hline 60 & 1 & 0 & 5.475808 & 1.806606 & 0.866625 \\
\hline 61 & 1 & 0 & 6.365146 & 3.330418 & 0.872157 \\
\hline 62 & 1 & 0 & 4.835806 & 3.196270 & 1.760225 \\
\hline 63 & 6 & 0 & 4.533687 & 4.920518 & -0.332947 \\
\hline 64 & 1 & 0 & 4.083846 & 5.353309 & -1.232943 \\
\hline 65 & 1 & 0 & 3.957578 & 5.256946 & 0.536415 \\
\hline 66 & 1 & 0 & 5.547039 & 5.328517 & -0.247077 \\
\hline 67 & 6 & 0 & 4.869974 & -2.328895 & -2.976481 \\
\hline
\end{tabular}




\begin{tabular}{|c|c|c|c|c|c|}
\hline 68 & 1 & 0 & 5.823591 & -2.781876 & -3.275444 \\
\hline 69 & 1 & 0 & 4.250784 & -2.227110 & -3.875764 \\
\hline 70 & 1 & 0 & 5.066741 & -1.335018 & -2.570828 \\
\hline 71 & 6 & 0 & 5.050460 & -3.439868 & -0.686012 \\
\hline 72 & 1 & 0 & 6.015414 & -3.872131 & -0.977877 \\
\hline 73 & 1 & 0 & 5.247997 & -2.506914 & -0.157769 \\
\hline 74 & 1 & 0 & 4.568627 & -4.128202 & 0.017635 \\
\hline 75 & 6 & 0 & 3.949932 & -4.617613 & -2.585365 \\
\hline 76 & 1 & 0 & 3.470513 & -5.322006 & -1.896145 \\
\hline 77 & 1 & 0 & 3.339527 & -4.549626 & -3.492729 \\
\hline 78 & 1 & 0 & 4.920820 & -5.038987 & -2.868196 \\
\hline 79 & 8 & 0 & -4.889333 & 0.461489 & 0.436463 \\
\hline 80 & 8 & 0 & 3.959354 & -0.693075 & 1.662966 \\
\hline 81 & 8 & 0 & 5.265888 & -0.043529 & -0.826040 \\
\hline 82 & 6 & 0 & 6.595897 & -0.234932 & -0.413324 \\
\hline 83 & 1 & 0 & 7.178225 & 0.687035 & -0.557378 \\
\hline 84 & 1 & 0 & 6.670278 & -0.522858 & 0.644977 \\
\hline 85 & 1 & 0 & 7.071606 & -1.023476 & -1.014148 \\
\hline 86 & 6 & 0 & 3.683398 & -0.098189 & 2.905140 \\
\hline 87 & 1 & 0 & 3.910635 & -0.812948 & 3.709382 \\
\hline 88 & 1 & 0 & 4.301317 & 0.797291 & 3.079742 \\
\hline 89 & 1 & 0 & 2.627425 & 0.190665 & 2.993164 \\
\hline 90 & 6 & 0 & -6.289896 & 0.506326 & 0.281987 \\
\hline 91 & 1 & 0 & -6.603689 & 0.402965 & -0.766033 \\
\hline 92 & 1 & 0 & -6.763670 & -0.302227 & 0.857455 \\
\hline 93 & 1 & 0 & -6.685649 & 1.459699 & 0.660654 \\
\hline 94 & 6 & 0 & -3.711682 & -0.176086 & -3.582614 \\
\hline 95 & 1 & 0 & -4.456677 & -0.051472 & -4.380731 \\
\hline 96 & 1 & 0 & -2.923737 & 0.576793 & -3.741716 \\
\hline 97 & 8 & 0 & -4.333875 & -0.044751 & -2.330436 \\
\hline 98 & 13 & 0 & 0.053419 & 0.060713 & 0.285120 \\
\hline 99 & 8 & 0 & -0.091088 & 1.564919 & 3.649695 \\
\hline 100 & 6 & 0 & -0.000386 & 1.468924 & 5.077031 \\
\hline 101 & 1 & 0 & -0.985187 & 1.384811 & 5.543403 \\
\hline 102 & 1 & 0 & 0.628733 & 0.621523 & 5.358689 \\
\hline 103 & 1 & 0 & 0.474635 & 2.397886 & 5.395344 \\
\hline 104 & 6 & 0 & 0.915796 & -2.093426 & 2.092440 \\
\hline 105 & 6 & 0 & 0.538703 & -2.500584 & 3.516676 \\
\hline
\end{tabular}




$\begin{array}{lrrrrr}106 & 6 & 0 & -0.978742 & -2.519661 & 3.802026 \\ 107 & 6 & 0 & -1.964133 & -0.077017 & 3.518054 \\ 108 & 6 & 0 & -1.593292 & -1.260070 & 4.442408 \\ 109 & 1 & 0 & 0.444950 & -2.815710 & 1.405281 \\ 110 & 1 & 0 & 1.048142 & -1.837446 & 4.231097 \\ 111 & 1 & 0 & -1.524380 & -2.763538 & 2.879714 \\ 112 & 1 & 0 & -2.547224 & -0.449681 & 2.672785 \\ 113 & 1 & 0 & 2.002867 & -2.182535 & 1.964915 \\ 114 & 1 & 0 & 0.947428 & -3.505781 & 3.690954 \\ 115 & 1 & 0 & -1.186328 & -3.341977 & 4.499422 \\ 116 & 1 & 0 & -2.638706 & 0.591668 & 4.077147 \\ 117 & 1 & 0 & -2.545126 & -1.555204 & 4.903272 \\ 118 & 1 & 0 & -0.957669 & -0.936572 & 5.272719 \\ 119 & 6 & 0 & -0.927502 & 0.846266 & 2.908310 \\ 120 & 8 & 0 & -0.975792 & 1.154879 & 1.707379 \\ 121 & 8 & 0 & 0.485837 & -0.781672 & 1.829360 \\ 122 & 1 & 0 & -3.253221 & -1.169860 & -3.707987\end{array}$


Table S30. Thermochemical data of cII

- Thermochemistry -

Temperature 298.150 Kelvin. Pressure 1.00000 Atm.

Molecular mass: $\quad 838.44225$ amu.

\begin{tabular}{|c|c|c|}
\hline & (Hartree/Particle) & $(\mathrm{kcal} / \mathrm{mol})$ \\
\hline Zero-point correction $=$ & 1.03419 & \\
\hline Thermal correction to Energy= & 1.099881 & \\
\hline Thermal correction to Enthalpy= & 1.100826 & \\
\hline Thermal correction to Gibbs Free Energy= & 0.93414 & \\
\hline Electronic Energy= & -3217.678332 & -2019123.638 \\
\hline Sum of electronic and zero-point Energies $=$ & -3216.644142 & -2018474.674 \\
\hline Sum of electronic and thermal Energies= & -3216.578451 & -2018433.452 \\
\hline Sum of electronic and thermal Enthalpies $=$ & -3216.577506 & -2018432.859 \\
\hline Sum of electronic and thermal Free Energies $=$ & $=-3216.744192$ & -2018537.456 \\
\hline E (Thermal) & $\mathrm{CV}$ & $\mathrm{S}$ \\
\hline $\mathrm{KCal} / \mathrm{Mol}$ & Cal/Mol-Kelvin & $\mathrm{Cal} / \mathrm{Mol}-\mathrm{Kelvin}$ \\
\hline 690.186 & 245.387 & 350.819 \\
\hline
\end{tabular}


Intermediate cIII

Table S31. Atomic coordinate of cIII

Standard orientation:

$\begin{array}{lcccc}\text { Center } & \text { Atomic } & \text { Atomic } & \text { Coordinates (Angstroms) } \\ \text { Number } & \text { Number } & \text { Type } & X & Y\end{array}$

\begin{tabular}{|c|c|c|c|c|c|}
\hline 1 & 7 & 0 & -0.660083 & 1.890142 & 0.337749 \\
\hline 2 & 6 & 0 & 0.031164 & 2.991621 & -0.001512 \\
\hline 3 & 6 & 0 & -2.125534 & 3.511553 & -0.031441 \\
\hline 4 & 6 & 0 & -0.848051 & 4.042954 & -0.237491 \\
\hline 5 & 1 & 0 & -0.599914 & 5.044245 & -0.550417 \\
\hline 6 & 7 & 0 & 1.845739 & 1.495766 & -0.086245 \\
\hline 7 & 6 & 0 & 1.463781 & 2.784886 & -0.118191 \\
\hline 8 & 6 & 0 & 3.675166 & 2.729982 & -0.279936 \\
\hline 9 & 6 & 0 & 2.581189 & 3.600822 & -0.238748 \\
\hline 10 & 1 & 0 & 2.601465 & 4.677723 & -0.278190 \\
\hline 11 & 7 & 0 & -0.929708 & -0.774115 & 1.265351 \\
\hline 12 & 6 & 0 & -0.454770 & -1.917271 & 1.787582 \\
\hline 13 & 6 & 0 & -1.502880 & -2.665880 & 2.308395 \\
\hline 14 & 6 & 0 & -2.654728 & -1.908236 & 2.070352 \\
\hline 15 & 1 & 0 & -1.443116 & -3.635811 & 2.774709 \\
\hline 16 & 7 & 0 & 1.580887 & -1.162630 & 0.881254 \\
\hline 17 & 6 & 0 & 0.980513 & -2.086537 & 1.650950 \\
\hline 18 & 6 & 0 & 3.157846 & -2.509302 & 1.662333 \\
\hline 19 & 6 & 0 & 1.931929 & -2.957788 & 2.165264 \\
\hline 20 & 1 & 0 & 1.762605 & -3.792425 & 2.825789 \\
\hline 21 & 7 & 0 & -2.290056 & -0.747968 & 1.437479 \\
\hline 22 & 7 & 0 & -1.997556 & 2.194118 & 0.325022 \\
\hline 23 & 7 & 0 & 3.213863 & 1.445430 & -0.182260 \\
\hline 24 & 7 & 0 & 2.931293 & -1.409574 & 0.878805 \\
\hline 25 & 13 & 0 & -3.161548 & 1.006995 & 1.362357 \\
\hline 26 & 13 & 0 & 3.923875 & -0.359028 & -0.447868 \\
\hline 27 & 6 & 0 & -4.084004 & -2.326825 & 2.406069 \\
\hline 28 & 6 & 0 & -3.448361 & 4.243631 & -0.230308 \\
\hline 29 & 6 & 0 & 4.519165 & -3.136886 & 1.943360 \\
\hline
\end{tabular}




\begin{tabular}{|c|c|c|c|c|c|}
\hline 30 & 6 & 0 & 5.136214 & 3.141284 & -0.424123 \\
\hline 31 & 6 & 0 & -4.728940 & -1.371662 & 3.435624 \\
\hline 32 & 1 & 0 & -4.817838 & -0.349048 & 3.068565 \\
\hline 33 & 1 & 0 & -5.734729 & -1.730410 & 3.684699 \\
\hline 34 & 1 & 0 & -4.140989 & -1.338846 & 4.359495 \\
\hline 35 & 6 & 0 & -4.927208 & -2.382693 & 1.110696 \\
\hline 36 & 1 & 0 & -5.951663 & -2.692626 & 1.350162 \\
\hline 37 & 1 & 0 & -4.960085 & -1.410023 & 0.615136 \\
\hline 38 & 1 & 0 & -4.507978 & -3.118027 & 0.413219 \\
\hline 39 & 6 & 0 & -4.067028 & -3.739958 & 3.028227 \\
\hline 40 & 1 & 0 & -3.636906 & -4.480356 & 2.344679 \\
\hline 41 & 1 & 0 & -3.503277 & -3.765702 & 3.967619 \\
\hline 42 & 1 & 0 & -5.093979 & -4.049326 & 3.250056 \\
\hline 43 & 6 & 0 & -4.201715 & 4.408550 & 1.109888 \\
\hline 44 & 1 & 0 & -4.418081 & 3.456697 & 1.597523 \\
\hline 45 & 1 & 0 & -3.612600 & 5.009683 & 1.812071 \\
\hline 46 & 1 & 0 & -5.152260 & 4.926484 & 0.936304 \\
\hline 47 & 6 & 0 & -4.312311 & 3.481142 & -1.262188 \\
\hline 48 & 1 & 0 & -3.795251 & 3.421950 & -2.227018 \\
\hline 49 & 1 & 0 & -4.535028 & 2.465363 & -0.929330 \\
\hline 50 & 1 & 0 & -5.257059 & 4.015626 & -1.418594 \\
\hline 51 & 6 & 0 & -3.172045 & 5.656277 & -0.788027 \\
\hline 52 & 1 & 0 & -2.579545 & 6.261606 & -0.092579 \\
\hline 53 & 1 & 0 & -2.647334 & 5.617827 & -1.749112 \\
\hline 54 & 1 & 0 & -4.123848 & 6.173875 & -0.948849 \\
\hline 55 & 6 & 0 & 5.455504 & -2.113293 & 2.626004 \\
\hline 56 & 1 & 0 & 5.012202 & -1.746175 & 3.559149 \\
\hline 57 & 1 & 0 & 6.408670 & -2.595876 & 2.874452 \\
\hline 58 & 1 & 0 & 5.660796 & -1.264719 & 1.970844 \\
\hline 59 & 6 & 0 & 5.144330 & -3.663026 & 0.631774 \\
\hline 60 & 1 & 0 & 5.325447 & -2.854078 & -0.076876 \\
\hline 61 & 1 & 0 & 6.106183 & -4.144735 & 0.843936 \\
\hline 62 & 1 & 0 & 4.491545 & -4.404082 & 0.156578 \\
\hline 63 & 6 & 0 & 4.341428 & -4.337276 & 2.897671 \\
\hline 64 & 1 & 0 & 3.912034 & -4.032146 & 3.858511 \\
\hline 65 & 1 & 0 & 3.702798 & -5.114749 & 2.463406 \\
\hline 66 & 1 & 0 & 5.320147 & -4.785686 & 3.099279 \\
\hline 67 & 6 & 0 & 5.962319 & 2.652156 & 0.787601 \\
\hline
\end{tabular}




\begin{tabular}{|c|c|c|c|c|c|}
\hline 68 & 1 & 0 & 6.997921 & 3.000340 & 0.690674 \\
\hline 69 & 1 & 0 & 5.557660 & 3.061149 & 1.721028 \\
\hline 70 & 1 & 0 & 5.969672 & 1.562629 & 0.852701 \\
\hline 71 & 6 & 0 & 5.709276 & 2.584684 & -1.747420 \\
\hline 72 & 1 & 0 & 6.756768 & 2.889124 & -1.858532 \\
\hline 73 & 1 & 0 & 5.670529 & 1.495370 & -1.780457 \\
\hline 74 & 1 & 0 & 5.149689 & 2.970909 & -2.606719 \\
\hline 75 & 6 & 0 & 5.232334 & 4.680950 & -0.477552 \\
\hline 76 & 1 & 0 & 4.686151 & 5.096224 & -1.331910 \\
\hline 77 & 1 & 0 & 4.848424 & 5.143682 & 0.438569 \\
\hline 78 & 1 & 0 & 6.282765 & 4.973258 & -0.581282 \\
\hline 79 & 8 & 0 & -4.705073 & 0.826464 & 0.613621 \\
\hline 80 & 8 & 0 & 3.435055 & -0.822006 & -2.029536 \\
\hline 81 & 8 & 0 & 5.609738 & -0.408392 & -0.084630 \\
\hline 82 & 6 & 0 & 6.751824 & -0.728765 & -0.841699 \\
\hline 83 & 1 & 0 & 7.270018 & -1.593276 & -0.403518 \\
\hline 84 & 1 & 0 & 6.508991 & -0.970680 & -1.886155 \\
\hline 85 & 1 & 0 & 7.455513 & 0.115187 & -0.842772 \\
\hline 86 & 6 & 0 & 3.028641 & -2.038803 & -2.610259 \\
\hline 87 & 1 & 0 & 3.030651 & -1.939365 & -3.703297 \\
\hline 88 & 1 & 0 & 3.701332 & -2.871935 & -2.352747 \\
\hline 89 & 1 & 0 & 2.010892 & -2.310284 & -2.299115 \\
\hline 90 & 6 & 0 & -6.007101 & 1.165798 & 1.025893 \\
\hline 91 & 1 & 0 & -6.031829 & 1.607388 & 2.031949 \\
\hline 92 & 1 & 0 & -6.452552 & 1.888528 & 0.327692 \\
\hline 93 & 1 & 0 & -6.647010 & 0.271891 & 1.033900 \\
\hline 94 & 6 & 0 & -2.246299 & 2.177836 & 3.803640 \\
\hline 95 & 1 & 0 & -2.701060 & 2.530819 & 4.739151 \\
\hline 96 & 1 & 0 & -1.484044 & 1.427441 & 4.067695 \\
\hline 97 & 8 & 0 & -3.241509 & 1.648953 & 2.966846 \\
\hline 98 & 13 & 0 & 0.338294 & 0.172061 & 0.039220 \\
\hline 99 & 8 & 0 & -4.061359 & -3.842873 & -4.016121 \\
\hline 100 & 6 & 0 & -5.197593 & -3.513392 & -3.216881 \\
\hline 101 & 1 & 0 & -5.121836 & -3.944078 & -2.211576 \\
\hline 102 & 1 & 0 & -5.350737 & -2.432452 & -3.138899 \\
\hline 103 & 1 & 0 & -6.053412 & -3.958832 & -3.728417 \\
\hline 104 & 6 & 0 & 0.180445 & 0.227437 & -2.796716 \\
\hline 105 & 6 & 0 & -0.383368 & -0.585539 & -3.965118 \\
\hline
\end{tabular}




$\begin{array}{lllrrr}106 & 6 & 0 & -1.893744 & -0.452593 & -4.223879 \\ 107 & 6 & 0 & -2.653863 & -2.347825 & -2.605562 \\ 108 & 6 & 0 & -2.832294 & -0.886686 & -3.085940 \\ 109 & 1 & 0 & -0.223055 & 1.254899 & -2.847982 \\ 110 & 1 & 0 & -0.115979 & -1.639794 & -3.829879 \\ 111 & 1 & 0 & -2.121187 & 0.598078 & -4.457685 \\ 112 & 1 & 0 & -1.641030 & -2.464979 & -2.215841 \\ 113 & 1 & 0 & 1.269871 & 0.300829 & -2.914087 \\ 114 & 1 & 0 & 0.136602 & -0.254099 & -4.875687 \\ 115 & 1 & 0 & -2.134444 & -1.024571 & -5.129446 \\ 116 & 1 & 0 & -3.349749 & -2.538707 & -1.782664 \\ 117 & 1 & 0 & -2.675837 & -0.245963 & -2.213143 \\ 118 & 1 & 0 & -3.869614 & -0.727055 & -3.411808 \\ 119 & 6 & 0 & -2.816413 & -3.359630 & -3.725433 \\ 120 & 8 & 0 & -1.895789 & -3.735190 & -4.415187 \\ 121 & 8 & 0 & -0.130290 & -0.361948 & -1.541721 \\ 122 & 1 & 0 & -1.726306 & 3.033288 & 3.343175 \\ ----------------------- & & \end{array}$


Table S32. Thermochemical data of cIII

- Thermochemistry -

Temperature 298.150 Kelvin. Pressure 1.00000 Atm.

Molecular mass: $\quad 838.44225 \mathrm{amu}$.

\begin{tabular}{|c|c|c|}
\hline & (Hartree/Particle) & $(\mathrm{kcal} / \mathrm{mol})$ \\
\hline Zero-point correction $=$ & 1.03406 & \\
\hline Thermal correction to Energy= & 1.100666 & \\
\hline Thermal correction to Enthalpy= & 1.10161 & \\
\hline Thermal correction to Gibbs Free Energy= & 0.928826 & \\
\hline Electronic Energy= & -3217.707387 & -2019141.870 \\
\hline Sum of electronic and zero-point Energies= & -3216.673327 & -2018492.988 \\
\hline Sum of electronic and thermal Energies $=$ & -3216.606721 & -2018451.192 \\
\hline Sum of electronic and thermal Enthalpies $=$ & -3216.605777 & -2018450.599 \\
\hline \multicolumn{2}{|l|}{ Sum of electronic and thermal Free Energies $=$} & -3216.778561 \\
\hline & -2018559.023 & \\
\hline E (Thermal) & $\mathrm{CV}$ & $\mathrm{S}$ \\
\hline KCal/Mol & Cal/Mol-Kelvin & Cal/Mol-Kelvin \\
\hline 690.678 & 245.358 & 363.654 \\
\hline
\end{tabular}


Dimer (cat)

Table S33. Atomic coordinates of (cat)

$\begin{array}{lcccc}\text { Center } & \text { Atomic } & \text { Atomic } & \text { Coordinates (Angstroms) } \\ \text { Number } & \text { Number } & \text { Type } & X & Y \\ Z & & & \end{array}$

\begin{tabular}{|c|c|c|c|c|c|}
\hline 1 & 13 & 0 & 3.362443 & -0.891621 & 1.468895 \\
\hline 2 & 7 & 0 & 4.532420 & 0.512179 & 0.655952 \\
\hline 3 & 6 & 0 & 4.119005 & 1.745534 & 0.992679 \\
\hline 4 & 6 & 0 & 6.181388 & 1.919096 & 0.194671 \\
\hline 5 & 6 & 0 & 5.124275 & 2.666194 & 0.722072 \\
\hline 6 & 1 & 0 & 5.093749 & 3.731405 & 0.883212 \\
\hline 7 & 7 & 0 & 2.081401 & 0.657634 & 1.510358 \\
\hline 8 & 6 & 0 & 2.797384 & 1.798287 & 1.587658 \\
\hline 9 & 6 & 0 & 0.969157 & 2.065091 & 2.813960 \\
\hline 10 & 6 & 0 & 2.135266 & 2.715152 & 2.387146 \\
\hline 11 & 1 & 0 & 2.479071 & 3.696165 & 2.671146 \\
\hline 12 & 7 & 0 & 4.409771 & -2.327693 & 0.528493 \\
\hline 13 & 6 & 0 & 3.834917 & -3.533589 & 0.681056 \\
\hline 14 & 6 & 0 & 4.786963 & -4.531498 & 0.501834 \\
\hline 15 & 6 & 0 & 5.982537 & -3.860498 & 0.225053 \\
\hline 16 & 1 & 0 & 4.638873 & -5.596368 & 0.580921 \\
\hline 17 & 7 & 0 & 1.929543 & -2.245230 & 1.215772 \\
\hline 18 & 6 & 0 & 2.423343 & -3.486151 & 1.010777 \\
\hline 19 & 6 & 0 & 0.252874 & -3.662815 & 1.414303 \\
\hline 20 & 6 & 0 & 1.404413 & -4.413798 & 1.130368 \\
\hline 21 & 1 & 0 & 1.476458 & -5.482399 & 1.008577 \\
\hline 22 & 7 & 0 & 5.742177 & -2.511938 & 0.243739 \\
\hline 23 & 7 & 0 & 5.812867 & 0.599198 & 0.165170 \\
\hline 24 & 7 & 0 & 0.929400 & 0.819217 & 2.246265 \\
\hline 25 & 7 & 0 & 0.585185 & -2.339888 & 1.457016 \\
\hline 26 & 13 & 0 & 6.554735 & -0.997545 & -0.689653 \\
\hline 27 & 13 & 0 & -0.470541 & -0.605120 & 1.888928 \\
\hline 28 & 6 & 0 & 7.337659 & -4.509776 & -0.032794 \\
\hline 29 & 6 & 0 & 7.528906 & 2.478388 & -0.250430 \\
\hline 30 & 6 & 0 & -1.136544 & -4.255303 & 1.629582 \\
\hline
\end{tabular}




\begin{tabular}{|c|c|c|c|c|c|}
\hline 31 & 6 & 0 & -0.018877 & 2.647953 & 3.826545 \\
\hline 32 & 6 & 0 & 7.759065 & -4.310119 & -1.506829 \\
\hline 33 & 1 & 0 & 7.845677 & -3.255823 & -1.771559 \\
\hline 34 & 1 & 0 & 8.732051 & -4.784554 & -1.682697 \\
\hline 35 & 1 & 0 & 7.030107 & -4.767997 & -2.185070 \\
\hline 36 & 6 & 0 & 8.398842 & -3.927529 & 0.928885 \\
\hline 37 & 1 & 0 & 9.360406 & -4.428550 & 0.762719 \\
\hline 38 & 1 & 0 & 8.533187 & -2.855125 & 0.773702 \\
\hline 39 & 1 & 0 & 8.107482 & -4.097977 & 1.972090 \\
\hline 40 & 6 & 0 & 7.235162 & -6.027994 & 0.227419 \\
\hline 41 & 1 & 0 & 6.931139 & -6.242675 & 1.258017 \\
\hline 42 & 1 & 0 & 6.524801 & -6.512996 & -0.451693 \\
\hline 43 & 1 & 0 & 8.215048 & -6.489931 & 0.065184 \\
\hline 44 & 6 & 0 & 7.795825 & 2.150959 & -1.736849 \\
\hline 45 & 1 & 0 & 7.820697 & 1.078642 & -1.933685 \\
\hline 46 & 1 & 0 & 7.020676 & 2.589589 & -2.375610 \\
\hline 47 & 1 & 0 & 8.762377 & 2.570646 & -2.040037 \\
\hline 48 & 6 & 0 & 8.654654 & 1.924468 & 0.654794 \\
\hline 49 & 1 & 0 & 8.480118 & 2.208329 & 1.699384 \\
\hline 50 & 1 & 0 & 8.715769 & 0.836343 & 0.594047 \\
\hline 51 & 1 & 0 & 9.618227 & 2.349783 & 0.348090 \\
\hline 52 & 6 & 0 & 7.516903 & 4.015610 & -0.107329 \\
\hline 53 & 1 & 0 & 6.738272 & 4.477514 & -0.724872 \\
\hline 54 & 1 & 0 & 7.365196 & 4.325745 & 0.932507 \\
\hline 55 & 1 & 0 & 8.482850 & 4.415890 & -0.433699 \\
\hline 56 & 6 & 0 & -2.203392 & -3.440834 & 0.887059 \\
\hline 57 & 1 & 0 & -1.979542 & -3.368318 & -0.182290 \\
\hline 58 & 1 & 0 & -3.182091 & -3.912982 & 1.005421 \\
\hline 59 & 1 & 0 & -2.307158 & -2.431572 & 1.287633 \\
\hline 60 & 6 & 0 & -1.487284 & -4.310319 & 3.135339 \\
\hline 61 & 1 & 0 & -1.553416 & -3.304464 & 3.554741 \\
\hline 62 & 1 & 0 & -2.456347 & -4.808050 & 3.266106 \\
\hline 63 & 1 & 0 & -0.736808 & -4.880529 & 3.694927 \\
\hline 64 & 6 & 0 & -1.166786 & -5.696767 & 1.073782 \\
\hline 65 & 1 & 0 & -0.895580 & -5.729802 & 0.012233 \\
\hline 66 & 1 & 0 & -0.492289 & -6.363065 & 1.622728 \\
\hline 67 & 1 & 0 & -2.179371 & -6.102340 & 1.176979 \\
\hline 68 & 6 & 0 & -1.378040 & 2.937346 & 3.167408 \\
\hline
\end{tabular}




\begin{tabular}{|c|c|c|c|c|c|}
\hline 69 & 1 & 0 & -2.095307 & 3.287271 & 3.919838 \\
\hline 70 & 1 & 0 & -1.281689 & 3.711311 & 2.402908 \\
\hline 71 & 1 & 0 & -1.786394 & 2.050390 & 2.687908 \\
\hline 72 & 6 & 0 & -0.203007 & 1.690225 & 5.022685 \\
\hline 73 & 1 & 0 & -0.851748 & 2.163210 & 5.770277 \\
\hline 74 & 1 & 0 & -0.655939 & 0.748874 & 4.710358 \\
\hline 75 & 1 & 0 & 0.758743 & 1.469156 & 5.499055 \\
\hline 76 & 6 & 0 & 0.536508 & 3.980100 & 4.377441 \\
\hline 77 & 1 & 0 & 1.495681 & 3.840311 & 4.888232 \\
\hline 78 & 1 & 0 & 0.670227 & 4.730380 & 3.589972 \\
\hline 79 & 1 & 0 & -0.172495 & 4.389155 & 5.105591 \\
\hline 80 & 8 & 0 & 8.264313 & -1.037008 & -0.470088 \\
\hline 81 & 8 & 0 & 6.088613 & -1.039952 & -2.351455 \\
\hline 82 & 8 & 0 & 3.828734 & -1.004221 & 3.138158 \\
\hline 83 & 8 & 0 & -0.729347 & -1.139113 & 3.559068 \\
\hline 84 & 8 & 0 & -2.263489 & -0.014010 & 1.661428 \\
\hline 85 & 6 & 0 & -3.219885 & -0.007631 & 2.748341 \\
\hline 86 & 1 & 0 & -3.774633 & 0.933200 & 2.748206 \\
\hline 87 & 1 & 0 & -3.910950 & -0.843579 & 2.638311 \\
\hline 88 & 1 & 0 & -2.669636 & -0.116660 & 3.680347 \\
\hline 89 & 6 & 0 & 0.283238 & -1.603546 & 4.424590 \\
\hline 90 & 1 & 0 & 0.597706 & -2.627777 & 4.178043 \\
\hline 91 & 1 & 0 & 1.183973 & -0.974312 & 4.399836 \\
\hline 92 & 1 & 0 & -0.095624 & -1.612562 & 5.457022 \\
\hline 93 & 6 & 0 & 5.084352 & -1.236336 & 3.717394 \\
\hline 94 & 1 & 0 & 5.797863 & -0.422155 & 3.508401 \\
\hline 95 & 1 & 0 & 4.977014 & -1.307130 & 4.808946 \\
\hline 96 & 1 & 0 & 5.542837 & -2.176149 & 3.369060 \\
\hline 97 & 6 & 0 & 9.357201 & -1.080225 & -1.352516 \\
\hline 98 & 1 & 0 & 10.005592 & -0.205608 & -1.198835 \\
\hline 99 & 1 & 0 & 9.961929 & -1.978979 & -1.164773 \\
\hline 100 & 1 & 0 & 9.048240 & -1.092440 & -2.407185 \\
\hline 101 & 6 & 0 & 4.850441 & -0.953184 & -3.004620 \\
\hline 102 & 1 & 0 & 4.331794 & -0.004616 & -2.791692 \\
\hline 103 & 1 & 0 & 5.002711 & -1.009476 & -4.091337 \\
\hline 104 & 1 & 0 & 4.172474 & -1.774658 & -2.722184 \\
\hline 105 & 13 & 0 & -2.488600 & 0.431749 & -0.205535 \\
\hline 106 & 7 & 0 & -2.385969 & 2.488680 & -0.148353 \\
\hline
\end{tabular}




\begin{tabular}{|c|c|c|c|c|c|}
\hline 107 & 6 & 0 & -3.465596 & 3.116381 & 0.342943 \\
\hline 108 & 6 & 0 & -1.926545 & 4.663283 & -0.035784 \\
\hline 109 & 6 & 0 & -3.223123 & 4.482466 & 0.446304 \\
\hline 110 & 1 & 0 & -3.890077 & 5.235775 & 0.837105 \\
\hline 111 & 7 & 0 & -4.480789 & 0.989982 & 0.275108 \\
\hline 112 & 6 & 0 & -4.616850 & 2.281180 & 0.600917 \\
\hline 113 & 6 & 0 & -6.525486 & 1.284412 & 1.104743 \\
\hline 114 & 6 & 0 & -5.883505 & 2.520942 & 1.121379 \\
\hline 115 & 1 & 0 & -6.281974 & 3.463134 & 1.460513 \\
\hline 116 & 7 & 0 & -1.978039 & 0.723293 & -2.253286 \\
\hline 117 & 6 & 0 & -2.225670 & -0.344462 & -3.021562 \\
\hline 118 & 6 & 0 & -1.508917 & -0.267947 & -4.209834 \\
\hline 119 & 6 & 0 & -0.765536 & 0.908849 & -4.118142 \\
\hline 120 & 1 & 0 & -1.512956 & -0.981388 & -5.017453 \\
\hline 121 & 7 & 0 & -3.505949 & -1.117201 & -1.190094 \\
\hline 122 & 6 & 0 & -3.111604 & -1.331931 & -2.454276 \\
\hline 123 & 6 & 0 & -4.576031 & -2.913933 & -1.961898 \\
\hline 124 & 6 & 0 & -3.729096 & -2.463646 & -2.972368 \\
\hline 125 & 1 & 0 & -3.585370 & -2.891653 & -3.951617 \\
\hline 126 & 7 & 0 & -1.062534 & 1.508923 & -2.914140 \\
\hline 127 & 7 & 0 & -1.434392 & 3.443703 & -0.433218 \\
\hline 128 & 7 & 0 & -5.658109 & 0.349828 & 0.587751 \\
\hline 129 & 7 & 0 & -4.468818 & -2.060695 & -0.892114 \\
\hline 130 & 13 & 0 & -0.434313 & 3.173149 & -2.098674 \\
\hline 131 & 13 & 0 & -5.794620 & -1.602639 & 0.459750 \\
\hline 132 & 6 & 0 & 0.279398 & 1.349566 & -5.149603 \\
\hline 133 & 6 & 0 & -1.171181 & 5.994433 & -0.020607 \\
\hline 134 & 6 & 0 & -5.449218 & -4.164430 & -2.062195 \\
\hline 135 & 6 & 0 & -7.968123 & 1.046366 & 1.545166 \\
\hline 136 & 6 & 0 & 0.088182 & 2.803959 & -5.627726 \\
\hline 137 & 1 & 0 & 0.158709 & 3.535265 & -4.821405 \\
\hline 138 & 1 & 0 & 0.856661 & 3.040603 & -6.373022 \\
\hline 139 & 1 & 0 & -0.890194 & 2.928428 & -6.106368 \\
\hline 140 & 6 & 0 & 1.692986 & 1.160295 & -4.548720 \\
\hline 141 & 1 & 0 & 2.451515 & 1.476019 & -5.275139 \\
\hline 142 & 1 & 0 & 1.814641 & 1.740715 & -3.633172 \\
\hline 143 & 1 & 0 & 1.871323 & 0.104932 & -4.310846 \\
\hline 144 & 6 & 0 & 0.171452 & 0.444386 & -6.397839 \\
\hline
\end{tabular}




\begin{tabular}{|c|c|c|c|c|c|}
\hline 145 & 1 & 0 & 0.382834 & -0.604807 & -6.165249 \\
\hline 146 & 1 & 0 & -0.819979 & 0.505704 & -6.860961 \\
\hline 147 & 1 & 0 & 0.907799 & 0.769895 & -7.140413 \\
\hline 148 & 6 & 0 & -1.786976 & 6.972621 & -1.047880 \\
\hline 149 & 1 & 0 & -1.639632 & 6.603471 & -2.064065 \\
\hline 150 & 1 & 0 & -2.859595 & 7.113558 & -0.872595 \\
\hline 151 & 1 & 0 & -1.301042 & 7.952207 & -0.961911 \\
\hline 152 & 6 & 0 & 0.330473 & 5.835049 & -0.308013 \\
\hline 153 & 1 & 0 & 0.785838 & 5.063918 & 0.321830 \\
\hline 154 & 1 & 0 & 0.510953 & 5.590007 & -1.356351 \\
\hline 155 & 1 & 0 & 0.839582 & 6.785143 & -0.108312 \\
\hline 156 & 6 & 0 & -1.327255 & 6.613595 & 1.391805 \\
\hline 157 & 1 & 0 & -2.376119 & 6.795116 & 1.647526 \\
\hline 158 & 1 & 0 & -0.897684 & 5.969595 & 2.165613 \\
\hline 159 & 1 & 0 & -0.806522 & 7.577529 & 1.425464 \\
\hline 160 & 6 & 0 & -6.791052 & -3.801180 & -2.742930 \\
\hline 161 & 1 & 0 & -6.615520 & -3.430024 & -3.759230 \\
\hline 162 & 1 & 0 & -7.428847 & -4.691081 & -2.814919 \\
\hline 163 & 1 & 0 & -7.315091 & -3.027575 & -2.179324 \\
\hline 164 & 6 & 0 & -5.684732 & -4.832119 & -0.693127 \\
\hline 165 & 1 & 0 & -6.208779 & -4.182367 & 0.007487 \\
\hline 166 & 1 & 0 & -6.298575 & -5.730051 & -0.828537 \\
\hline 167 & 1 & 0 & -4.737444 & -5.137058 & -0.236288 \\
\hline 168 & 6 & 0 & -4.722223 & -5.203771 & -2.949833 \\
\hline 169 & 1 & 0 & -4.600324 & -4.853483 & -3.979658 \\
\hline 170 & 1 & 0 & -3.732258 & -5.452246 & -2.551719 \\
\hline 171 & 1 & 0 & -5.314741 & -6.124903 & -2.988860 \\
\hline 172 & 6 & 0 & -8.848899 & 0.764150 & 0.304586 \\
\hline 173 & 1 & 0 & -9.887522 & 0.594274 & 0.614633 \\
\hline 174 & 1 & 0 & -8.837835 & 1.626571 & -0.372107 \\
\hline 175 & 1 & 0 & -8.493953 & -0.109812 & -0.243846 \\
\hline 176 & 6 & 0 & -8.064736 & -0.095660 & 2.580025 \\
\hline 177 & 1 & 0 & -9.107742 & -0.221445 & 2.893646 \\
\hline 178 & 1 & 0 & -7.720417 & -1.051760 & 2.186693 \\
\hline 179 & 1 & 0 & -7.467291 & 0.134470 & 3.469235 \\
\hline 180 & 6 & 0 & -8.511296 & 2.321642 & 2.228302 \\
\hline 181 & 1 & 0 & -7.916101 & 2.600937 & 3.105032 \\
\hline 182 & 1 & 0 & -8.536982 & 3.173512 & 1.540380 \\
\hline
\end{tabular}




\begin{tabular}{|c|c|c|c|c|c|}
\hline 183 & 1 & 0 & -9.538192 & 2.140333 & 2.563855 \\
\hline 184 & 8 & 0 & 1.256265 & 2.984905 & -1.848744 \\
\hline 185 & 8 & 0 & -0.945806 & 4.486498 & -3.120509 \\
\hline 186 & 8 & 0 & -0.684103 & -0.229575 & 0.010179 \\
\hline 187 & 8 & 0 & -5.369308 & -2.290444 & 1.978673 \\
\hline 188 & 8 & 0 & -7.358950 & -2.009329 & -0.170281 \\
\hline 189 & 6 & 0 & -8.431035 & -2.784043 & 0.301776 \\
\hline 190 & 1 & 0 & -8.695332 & -3.558999 & -0.431626 \\
\hline 191 & 1 & 0 & -8.210563 & -3.289209 & 1.253819 \\
\hline 192 & 1 & 0 & -9.320876 & -2.157621 & 0.456422 \\
\hline 193 & 6 & 0 & -5.779221 & -3.244197 & 2.913266 \\
\hline 194 & 1 & 0 & -6.710058 & -2.947636 & 3.422714 \\
\hline 195 & 1 & 0 & -5.949023 & -4.232270 & 2.456876 \\
\hline 196 & 1 & 0 & -5.005434 & -3.364829 & 3.684014 \\
\hline 197 & 6 & 0 & 0.354782 & -0.399400 & -0.968267 \\
\hline 198 & 1 & 0 & 0.869546 & 0.546357 & -1.150770 \\
\hline 199 & 1 & 0 & 1.073322 & -1.131287 & -0.605962 \\
\hline 200 & 1 & 0 & -0.063114 & -0.779980 & -1.900243 \\
\hline 201 & 6 & 0 & 2.420754 & 3.754218 & -1.980577 \\
\hline 202 & 1 & 0 & 3.241349 & 3.128293 & -2.358604 \\
\hline 203 & 1 & 0 & 2.294396 & 4.596253 & -2.677410 \\
\hline 204 & 1 & 0 & 2.736974 & 4.161123 & -1.009949 \\
\hline 205 & 6 & 0 & -2.185566 & 4.612303 & -3.778029 \\
\hline 206 & 1 & 0 & -2.987730 & 4.907000 & -3.083587 \\
\hline 207 & 1 & 0 & -2.114988 & 5.387972 & -4.552762 \\
\hline 208 & 1 & 0 & -2.498099 & 3.678119 & -4.268112 \\
\hline
\end{tabular}


Table S34. Thermochemical data of (cat)

- Thermochemistry -

Temperature 298.150 Kelvin. Pressure 1.00000 Atm.

Molecular mass: $1448.74833 \mathrm{amu}$.

\begin{tabular}{|c|c|c|}
\hline & (Hartree/Particle) & $(\mathrm{kcal} / \mathrm{mol})$ \\
\hline Zero-point correction $=$ & 1.755329 & \\
\hline Thermal correction to Energy= & 1.869533 & \\
\hline Thermal correction to Enthalpy= & 1.870477 & \\
\hline Thermal correction to Gibbs Free Energy= & 1.604812 & \\
\hline Electronic Energy= & -5665.158705 & -3554940.759 \\
\hline Sum of electronic and zero-point Energies= & -5663.403376 & -3553839.273 \\
\hline Sum of electronic and thermal Energies= & -5663.289172 & -3553767.609 \\
\hline Sum of electronic and thermal Enthalpies $=$ & -5663.288228 & -3553767.017 \\
\hline \multicolumn{2}{|c|}{ Sum of electronic and thermal Free Energies $=$} & -5663.553893 \\
\hline & -3553933.724 & \\
\hline E (Thermal) & $\mathrm{CV}$ & $\mathrm{S}$ \\
\hline $\mathrm{KCal} / \mathrm{Mol}$ & Cal/Mol-Kelvin & Cal/Mol-Kelvin \\
\hline 1173.149 & 428.984 & 559.139 \\
\hline
\end{tabular}


Table 36. Selected bond lengths $(\AA)$ and bond angles (deg) of $\mathbf{D}^{\mathbf{B u}}{ }_{2} \mathbf{A l}_{\mathbf{3}} \mathbf{M} \mathbf{e}_{\mathbf{5}}$

\begin{tabular}{c|ccc}
$\begin{array}{c}\text { Bond } \\
\text { lengths }\end{array}$ & $\mathrm{Al}(1)$ & $\mathrm{Al}(2)$ & $\mathrm{Al}(3)$ \\
\hline $\mathrm{Al}-\mathrm{C}$ & $\mathrm{Al}(1)-\mathrm{C}(1) 1.960(3)$ & $\mathrm{Al}(2)-\mathrm{C}(3) 1.942(3)$ & $\mathrm{Al}(3)-\mathrm{C}(4) 1.974(3)$ \\
& $\mathrm{Al}(1)-\mathrm{C}(2) 1.969(3)$ & $\mathrm{Al}(3)-\mathrm{C}(5) 1.960(3)$ \\
$\mathrm{Al}-\mathrm{N}$ & $\mathrm{Al}(1)-\mathrm{N}(1) 1.979(2)$ & $\mathrm{Al}(2)-\mathrm{N}(2) 1.977(2)$ & $\mathrm{Al}(3)-\mathrm{N}(4) 1.967(2)$ \\
& $\mathrm{Al}(1)-\mathrm{N}(8) 1.977(2)$ & $\mathrm{Al}(2)-\mathrm{N}(3) 1.997(2)$ & $\mathrm{Al}(3)-\mathrm{N}(5) 1.972(2)$ \\
& & $\mathrm{Al}(2)-\mathrm{N}(6) 2.002(2)$ & \\
& & $\mathrm{Al}(2)-\mathrm{N}(7) 1.976(2)$ & $\mathrm{Al}(3)$ \\
\hline Bond & $\mathrm{Al}(1)$ & $\mathrm{Al}(2)$ & \\
angles & & $\mathrm{C}(4)-\mathrm{Al}(3)-\mathrm{C}(5) 109.30(13)$ \\
$\angle \mathrm{C}-\mathrm{Al}-\mathrm{C}$ & $\mathrm{C}(1)-\mathrm{Al}(1)-\mathrm{C}(2) 123.96(12)$ & $\mathrm{N}(4)-\mathrm{Al}(3)-\mathrm{N}(5) 99.55(8)$ \\
& $\mathrm{N}-\mathrm{Al}-\mathrm{N}$ & $\mathrm{N}(1)-\mathrm{Al}(1)-\mathrm{N}(8) 106.01(8)-\mathrm{Al}(2)-\mathrm{N}(7) 89.85(8)$ & \\
& & $\mathrm{N}(7)-\mathrm{Al}(2)-\mathrm{N}(6) 80.36(8)$ & \\
& & $\mathrm{N}(6)-\mathrm{Al}(2)-\mathrm{N}(3) 88.19(8)$ & \\
& & $\mathrm{N}(3)-\mathrm{Al}(2)-\mathrm{N}(2) 80.32(8)$ &
\end{tabular}

